This item was submitted to Loughborough's Research Repository by the author.

Items in Figshare are protected by copyright, with all rights reserved, unless otherwise indicated.

\title{
Hodge theory for intersection space cohomology
}

PLEASE CITE THE PUBLISHED VERSION

https://doi.org/10.2140/gt.2019.23.2165

PUBLISHER

Mathematical Sciences Publishers

VERSION

VoR (Version of Record)

PUBLISHER STATEMENT

First published in Geometry \& Topology in Vol. 23 (2018), No. 5, published by Mathematical Sciences Publishers.

\section{LICENCE}

CC BY-NC-ND 4.0

\section{REPOSITORY RECORD}

Banagl, Markus, and Eugenie Hunsicker. 2019. "Hodge Theory for Intersection Space Cohomology”. figshare. https://hdl.handle.net/2134/36213. 


\title{
Hodge theory for intersection space cohomology
}

\author{
MARKUS BANAGL \\ EUGÉNIE HUNSICKER
}

\begin{abstract}
Given a perversity function in the sense of intersection homology theory, the method of intersection spaces assigns to certain oriented stratified spaces cell complexes whose ordinary reduced homology with real coefficients satisfies Poincaré duality across complementary perversities. The resulting homology theory is well known not to be isomorphic to intersection homology. For a two-strata pseudomanifold with product link bundle, we give a description of the cohomology of intersection spaces as a space of weighted $L^{2}$ harmonic forms on the regular part, equipped with a fibred scattering metric. Some consequences of our methods for the signature are discussed as well.
\end{abstract}

$55 \mathrm{~N} 33,58 \mathrm{~A} 14$

\section{Introduction}

Classical approaches to Poincaré duality on singular spaces are Cheeger's $L^{2}$ cohomology with respect to suitable conical metrics on the regular part of the space $[14 ; 13 ; 15]$ and Goresky and MacPherson's intersection homology, depending on a perversity parameter. Cheeger's Hodge theorem asserts that the space of $L^{2}$ harmonic forms on the regular part is isomorphic to the linear dual of intersection homology for the middle perversity, at least when $X$ has only strata of even codimension or, more generally, is a so-called Witt space.

More recently, the first author has introduced and investigated a different, spatial perspective on Poincaré duality for singular spaces [2]. This approach associates to certain classes of singular spaces $X$ a cell complex $I^{\bar{p}} X$, which depends on a perversity $\bar{p}$ and is called an intersection space of $X$. Intersection spaces are required to be generalized geometric Poincaré complexes in the sense that when $X$ is closed and oriented, there is a Poincaré duality isomorphism $\widetilde{H}^{i}\left(I^{\bar{p}} X ; \mathbb{R}\right) \cong \widetilde{H}_{n-i}\left(I^{\bar{q}} X ; \mathbb{R}\right)$, where $n$ is the dimension of $X, \bar{p}$ and $\bar{q}$ are complementary perversities in the sense of intersection homology theory, and $\widetilde{H}^{*}$ and $\widetilde{H}_{*}$ denote reduced singular (or cellular) cohomology and homology, respectively. The present paper is concerned with $X$ 
that have two strata such that the bottom stratum has a trivializable link bundle. The construction of intersection spaces for such $X$, first given in [2, Chapter 2.9], is described here in more detail in Section 3. The fundamental principle, even for more general $X$ (see Banagl and Chriestenson [7]), is to replace links by their Moore approximations, a concept from homotopy theory Eckmann-Hilton dual to the concept of Postnikov approximations. The resulting (co)homology theory $H I_{\bar{p}}^{*}(X)=H^{*}\left(I^{\bar{p}} X ; \mathbb{R}\right)$ or $H I_{*}^{\bar{p}}(X)=H_{*}\left(I^{\bar{p}} X ; \mathbb{R}\right)$ is not isomorphic to the respective intersection (co)homology $I H_{\bar{p}}^{*}(X ; \mathbb{R})$ or $I H_{*}^{\bar{p}}(X ; \mathbb{R})$. The theory $H I^{*}$ has had applications in fibre bundle theory and computation of equivariant cohomology (Banagl [3]); $K$-theory (Banagl [2, Chapter 2.8]; Spiegel [35]); algebraic geometry (smooth deformation of singular varieties (Banagl and Maxim [8; 9]), perverse sheaves (Banagl, Budur and Maxim [5]), mirror symmetry [2, Chapter 3.8]); and theoretical physics [2, Chapter 3; 5]. Note for example that the approach of intersection spaces makes it straightforward to define intersection $K$-groups by $K^{*}\left(I^{\bar{p}} X\right)$. These techniques are not accessible to classical intersection cohomology. There are also applications to Sullivan formality of singular spaces: Given a perversity $\bar{p}$, call a pseudomanifold $X \bar{p}$-intersection formal if $I^{\bar{p}} X$ is formal in the usual sense. Then the results of [8] show that under a mild torsionfreeness hypothesis on the homology of links, complex projective hypersurfaces $X$ with only isolated singularities, whose monodromy operators on the cohomology of the associated Milnor fibres are trivial, are middle-perversity $(\bar{m})$ intersection formal, since there is an algebra isomorphism from $H I_{\bar{m}}^{*}(X)$ to the ordinary cohomology algebra of a nearby smooth deformation, which is formal, being a Kähler manifold. This agrees nicely with the result of Chataur, Saralegi-Aranguren and Tanré [12, Section 3.4], where it is shown that any nodal hypersurface in $\mathbb{C P}^{4}$ is "totally" (ie with respect to an algebra that involves all perversities at once) intersection formal. Rational Sullivan models of intersection spaces have been investigated by M Klimczak [27].

A de Rham description of $H I_{\bar{p}}^{*}(X)$ has been given by Banagl [4] for two-strata spaces whose link bundle is flat with respect to the isometry group of the link. Under this assumption, a subcomplex $\Omega I_{\bar{p}}^{*}(M)$ of the complex $\Omega^{*}(M)$ of all smooth differential forms on the top stratum $M=X-\Sigma$, where $\Sigma \subset X$ is the singular set, has been defined such that for isolated singularities there is a de Rham isomorphism $H I_{\mathrm{dR}, \bar{p}}^{*}(X) \cong \widetilde{H}^{*}\left(I^{\bar{p}} X ; \mathbb{R}\right)$, where $H I_{\mathrm{dR}, \bar{p}}^{j}(X)=H^{j}\left(\Omega I_{\bar{p}}^{*}(M)\right)$. This result has been generalized by Timo Essig [16] to two-strata spaces with product link bundle. In [4] we prove furthermore that wedge product followed by integration over $M$ induces a nondegenerate intersection pairing $\cap_{H I}: H I_{\mathrm{dR}, \bar{p}}^{j}(X) \otimes H I_{\mathrm{dR}, \bar{q}}^{n-j}(X) \rightarrow \mathbb{R}$ for 
complementary $\bar{p}$ and $\bar{q}$. The construction of $\Omega I_{\bar{p}}^{*}(M)$ for the case of a product link bundle (ie the case relevant to this paper) is reviewed here in detail in Section 6.2.

In the present paper, we find for every perversity $\bar{p}$ a Hodge-theoretic description of the theory $H I_{\bar{p}}^{*}(X)$; that is, we find a Riemannian metric on $M$ (which is very different from Cheeger's class of metrics) and a suitable space of $L^{2}$ harmonic forms with respect to this metric (the extended weighted $L^{2}$ harmonic forms for suitable weights) such that the latter space is isomorphic to $\widetilde{H} I_{\bar{p}}^{*}(X) \cong H I_{\mathrm{dR}, \bar{p}}^{j}(X)$. Assume for simplicity that $\Sigma$ is connected. If $L$ denotes the link of $\Sigma$ in $X$ and $\bar{M}$ is the compact manifold with boundary $\partial \bar{M}=L \times \Sigma$ and interior $M$ (called the "blowup" of $X$ ), then a metric $g_{\mathrm{fs}}$ on $M$ is called a product-type fibred scattering metric if near $\partial \bar{M}$ it has the form

$$
g_{\mathrm{fs}}=\frac{d x^{2}}{x^{4}}+g_{\Sigma}+\frac{g_{L}}{x^{2}}
$$

where $g_{L}$ is a metric on the link and $g_{\Sigma}$ a metric on the singular set; see Section 2. If $\Sigma$ is a point, then $g_{\mathrm{fs}}$ is a scattering metric.

Given a weight $c$, weighted $L^{2}$ spaces $x^{c} L_{g_{\mathrm{fs}}}^{2} \Omega_{g_{\mathrm{fs}}}^{*}(M)$ are defined in Section 7 . The space $\mathcal{H}_{\text {ext }}^{*}\left(M, g_{\mathrm{fs}}, c\right)$ of extended weighted $L^{2}$ harmonic forms on $M$ consists of all those forms $\omega$ which are in the kernel of $d+\delta$ (where $\delta$ is the formal adjoint of the exterior derivative $d$ and depends on $g_{\mathrm{fs}}$ and $c$ ) and in $x^{c-\epsilon} L_{g_{\mathrm{fs}}}^{2} \Omega_{g_{\mathrm{fs}}}^{*}(M)$ for every $\epsilon>0$; see Definition 7.1. Extended $L^{2}$ harmonic forms are already present in Chapter 6.4 of Melrose's monograph [30]. Then our Hodge theorem is:

Theorem 1.1 Let $X$ be a (Thom-Mather) stratified pseudomanifold with smooth, connected singular stratum $\Sigma \subset X$. Assume that the link bundle $Y \rightarrow \Sigma$ is a product $L \times \Sigma \rightarrow \Sigma$, where $L$ is a smooth manifold of dimension $l$. Let $g_{\mathrm{fs}}$ be an associated product-type fibred scattering metric on $M=X-\Sigma$. Then

$$
H I_{\mathrm{dR}, \bar{p}}^{*}(X) \cong \mathcal{H}_{\mathrm{ext}}^{*}\left(M, g_{\mathrm{fs}}, \frac{1}{2}(l-1)-\bar{p}(l+1)\right) .
$$

Aside from giving an analytic description of $H I$ cohomology in terms of harmonic forms, a nice aspect of this result is that it shows that there is a natural topological description of the space of extended harmonic forms on the right-hand side. In [25], the second author obtained a Hodge theorem for these spaces of forms, but thought of as relating to a conformally equivalent metric and with a different weight. However, the topological description in that paper is in terms of the topologically less natural $I G$ spaces we consider below. Thus it is satisfying here to see that there is a natural cohomology describing these forms. 
As a corollary to this description, we find that the spaces $\mathcal{H}_{\text {ext }}^{*}\left(M, g_{\mathrm{fs}}, \frac{1}{2}(l-1)-\bar{p}(l+1)\right)$ satisfy Poincaré duality across complementary perversities. We can see this duality achieved on forms using an appropriate Hodge star operator, as has been shown by the second author in [25]. Finally, it is worth noting that on the space of extended harmonic forms, integration does not give a natural well-defined intersection pairing on the right-hand side. Thus it would be interesting in the future to consider how to realize the intersection pairing on extended harmonic forms.

The strategy of the proof of Theorem 1.1 is as follows: First we relate $H I_{\mathrm{dR}, \bar{p}}^{*}(X)$ and $\widetilde{H}_{*}\left(I^{\bar{p}} X ; \mathbb{R}\right)$ to intersection cohomology and intersection homology, respectively. To do this, we introduce in Section 2 the device of a conifold transition $\mathrm{CT}(X)$ associated to an $X$ as in the Hodge theorem. The conifold transition arose originally in theoretical physics and algebraic geometry as a means of connecting different Calabi-Yau 3-folds to each other by a process of deformations and small resolutions; see [2, Chapter 3] for more information. Topologically, such a process also arises in manifold surgery theory when $\Sigma$ is an embedded sphere with trivial normal bundle. The relation of $H I$ to $I H$ is then given by the following theorem.

Theorem 1.2 (homological version) Let $X$ be an $n$-dimensional stratified pseudomanifold with smooth nonempty singular stratum $\Sigma \subset X$. Assume that $\Sigma$ is closed as a manifold and the link bundle $Y \rightarrow \Sigma$ is a product bundle $L \times \Sigma \rightarrow \Sigma$, where the link $L$ is a smooth closed manifold of dimension $l$. Then the reduced homology $\tilde{H} I_{*}^{\bar{p}}(X)$ of the intersection space of perversity $\bar{p}$ is related to the intersection homology of the conifold transition $\mathrm{CT}(X)$ of $X$ by

$$
\tilde{H} I_{j}^{\bar{p}}(X) \cong I G_{j}^{(n-1-\bar{p}(l+1)-j)}(\mathrm{CT}(X)),
$$

where for a pseudomanifold $W$ with one singular stratum of codimension $c$,

$$
I G_{j}^{(k)}(W)=I H_{j}^{\bar{q}}(W) \oplus \frac{I H_{j}^{\bar{q}^{\prime}}(W)}{\operatorname{im}\left(I H_{j}^{\bar{q}}(W) \rightarrow I H_{j}^{\bar{q}^{\prime}}(W)\right)},
$$

with $\bar{q}(c)=k-1$ and $\bar{q}^{\prime}(c)=k$.

Note that when both $\bar{p}(l+1)$ and the degree $j$ are large, one must allow negative values for $k$ in the quotient $I G_{j}^{(k)}$. Therefore, the perversity functions $\bar{q}$ considered in this paper are not required to satisfy the Goresky-MacPherson conditions, but are simply arbitrary integer-valued functions. This, in turn, necessitates a minor modification in the definition of intersection homology. The precise definition of $I H_{*}^{\bar{q}}$ used in the 
above theorem is provided in Section 4 and has been introduced independently by Saralegi-Aranguren [31] and by Friedman [17]. In the following de Rham version of the above result, the link and the singular stratum are assumed to be orientable, as our methods rely on the availability of the Hodge star operator. When an intersection cohomology group $I H_{\bar{p}}^{*}(W)$ depends only on the value of the perversity $\bar{p}$ at one particular codimension, it will frequently be more convenient to label the group directly by the cutoff degree determined by the perversity. Thus we use the notation $I H_{(q)}^{j}(W)$ to mean that the integer $q$ is the cutoff degree in the local cohomology calculation on the link. The Poincaré lemma for a cone on a closed manifold $L$ then has the form

$$
I H_{(q)}^{j}\left(c^{\circ} L\right)=\left\{\begin{array}{cl}
H^{j}(L) & \text { if } j<q \\
0 & \text { if } j \geq q .
\end{array}\right.
$$

Theorem 1.3 (De Rham cohomological version) Let $X$ be an $n$-dimensional stratified pseudomanifold with smooth nonempty singular stratum $\Sigma \subset X$. Assume that $\Sigma$ is closed and orientable, and the link bundle $Y \rightarrow \Sigma$ is a product bundle $L \times \Sigma \rightarrow \Sigma$, where the link $L$ is a smooth closed orientable manifold of dimension $l$. Then the de Rham cohomology $H I_{\mathrm{dR}, \bar{p}}^{*}(X)$ can be described in terms of the intersection cohomology of the conifold transition by

$$
H I_{\mathrm{dR}, \bar{p}}^{j}(X) \cong I G_{(j+1-k)}^{j}(\mathrm{CT}(X)),
$$

where $k=l-\bar{p}(l+1)$ and for a pseudomanifold $W$ with one singular stratum,

$$
I G_{(q)}^{j}(W)=I H_{(q-1)}^{j}(W) \oplus \frac{I H_{(q)}^{j}(W)}{\operatorname{im}\left(I H_{(q-1)}^{j}(W) \rightarrow I H_{(q)}^{j}(W)\right)} .
$$

We do not deduce the cohomological version from the homological one by universal coefficient theorems, but prefer to give independent proofs for each version. The proof of the homological version uses Mayer-Vietoris techniques while the proof of the cohomological version compares differential forms in the various de Rham complexes on $M$. (The regular part of the conifold transition coincides with the regular part $M$ of $X$.) Finally, we appeal to a result (Theorem 7.2 in the present paper) of the second author [25], which relates extended weighted $L^{2}$ harmonic forms with respect to a fibred cusp metric $g_{\mathrm{fc}}$ to the $I G_{(q)}^{j}$ arising in Theorem 1.3 above. This leads in a natural way to fibred scattering metrics because fibred cusp metrics

$$
g_{\mathrm{fc}}=\frac{d x^{2}}{x^{2}}+g_{L}+x^{2} g_{\Sigma}
$$


on the conifold transition are conformal to

$$
\frac{1}{x^{2}} g_{\mathrm{fc}}=\frac{d x^{2}}{x^{4}}+\frac{1}{x^{2}} g_{L}+g_{\Sigma}
$$

which is precisely a fibred scattering metric on $X$.

For $n$ divisible by 4 , and either $l$ odd or $H^{l / 2}(L)=0$ (ie $X$ a Witt space), the nondegenerate intersection pairing $\cap_{H I}: H I_{\mathrm{dR}, \bar{m}}^{n / 2}(X) \otimes H I_{\mathrm{dR}, \bar{m}}^{n / 2}(X) \rightarrow \mathbb{R}$ on the middle dimension $n / 2$ for the middle perversity has a signature $\sigma_{H I}(X)$. It has been shown in [2, Theorem 2.28] that in the setting of isolated singularities, the signature of the homological intersection form $H_{n / 2}\left(I^{\bar{m}} X\right) \otimes H_{n / 2}\left(I^{\bar{m}} X\right) \rightarrow \mathbb{R}$ is equal to the Goresky-MacPherson signature coming from intersection homology of $X$. Banagl and Chriestenson [7, Theorem 11.3 and Corollary 11.4] generalized this result to a class of nonisolated singularities. We use Theorem 1.3 to obtain results about the intersection pairing and signature on $H I_{\mathrm{dR}}^{*}(X)$. This turns out to be related to perverse signatures of the conifold transition, which needs not be Witt even when $X$ is. Perverse signatures are defined for arbitrary perversities on arbitrary compact oriented pseudomanifolds from the extended intersection pairing on intersection cohomology. These signatures are defined in the two-strata case by Hunsicker in [24] and more generally by Friedman and Hunsicker in [18].

Theorem 1.4 Let $n$ be divisible by 4 and let $X$ be an $n$-dimensional compact oriented stratified pseudomanifold with smooth singular stratum $\Sigma \subset X$. Assume that the link bundle $Y \rightarrow \Sigma$ is a product bundle $L \times \Sigma \rightarrow \Sigma$. Then the intersection pairing $\cap_{H I}: H I_{\mathrm{dR}, \bar{p}}^{j} \otimes H I_{\mathrm{dR}, \bar{q}(X)}^{n-j} \rightarrow \mathbb{R}$ for dual perversities $\bar{p}$ and $\bar{q}$ is compatible with the intersection pairing on the intersection cohomology spaces $I G^{*}$ appearing in Theorem 1.3. When $X$ is an even-dimensional Witt space, then the signature $\sigma_{H I}(X)$ of the intersection form on $H I_{\mathrm{dR}, \bar{m}}^{n / 2}(X)$ is equal both to the signature $\sigma_{I H}(X)$ of the Goresky-MacPherson intersection form on $I_{\bar{m}}^{n / 2}(X)$, and to the perverse signature $\sigma_{I H, \bar{m}}(\mathrm{CT}(X))$, that is, the signature of the intersection form on

$$
\operatorname{im}\left(I H_{\bar{m}}^{n / 2}(\mathrm{CT}(X)) \rightarrow I H_{\bar{n}}^{n / 2}(\mathrm{CT}(X))\right),
$$

where $\bar{m}$ is the lower-middle and $\bar{n}$ the upper-middle perversity. Further,

$$
\sigma_{H I}(X)=\sigma_{I H}(X)=\sigma_{I H, \bar{m}}(\mathrm{CT}(X))=\sigma_{I H}(Z)=\sigma_{H I}(Z)=\sigma(\bar{M}),
$$

where $Z$ is the one-point compactification of $X-\Sigma$ and $\sigma(\bar{M})$ is the Novikov signature of the complement $\bar{M}$ of an open tubular neighborhood of the singular set. 
Remark 1.5 The compactification $Z$ appearing in Theorem 1.4 has one isolated singular point. Since $X$ is even-dimensional, $Z$ is thus a Witt space and has a welldefined $I H$-signature and a well-defined $H I$-signature. However, if $X$ satisfies the Witt condition, then $\mathrm{CT}(X)$ need not satisfy the Witt condition and $\sigma_{I H}(\mathrm{CT}(X))$ and $\sigma_{H I}(\mathrm{CT}(X))$ are a priori not defined. Therefore, we must use the perverse signature $\sigma_{I H, \bar{m}}$ for $\mathrm{CT}(X)$ as defined in $[24 ; 18]$.

We prove Theorem 1.4 using de Rham theory, Siegel's work [33], Novikov additivity and results of Banagl, Cappell and Shaneson [6]. Using different, algebraic methods and building on results of [2], parts of this theorem were also obtained by Matthias Spiegel in his dissertation [35].

Notation If $f$ is a continuous map, then cone $(f)$ denotes its mapping cone. For a compact topological space $X$, we denote by $c X$ the closed cone and by $c^{\circ} X$ the open cone on $X$. Only homology and cohomology with real coefficients are used in this paper. Thus we will write $H_{*}(X)=H_{*}(X ; \mathbb{R})$. When $M$ is a smooth manifold, $H^{*}(M)$ is generally, unless indicated otherwise, understood to mean de Rham cohomology. The symbol $\widetilde{H}_{*}(X)$ denotes the reduced (singular) homology of $X$, and $\widetilde{H}^{*}(X)$ denotes the reduced cohomology.

\section{The conifold transition and Riemannian metrics}

Let $X$ be a Thom-Mather stratified pseudomanifold with a single compact smooth singular stratum, $\Sigma$. Let $M=X-\Sigma$. Assume that the link bundle of $\Sigma$ is a product, $L \times \Sigma$. Let $N \subset X$ be an open tubular neighborhood of $\Sigma$. Fix a diffeomorphism

$$
\theta: N-\Sigma \cong L \times \Sigma \times(0,1)
$$

that extends to a homeomorphism

Define the blowup

$$
\tilde{\theta}: N \cong \frac{L \times \Sigma \times[0,1)}{(z, y, 0) \sim\left(z^{\prime}, y, 0\right)} \cong c^{\circ}(L) \times \Sigma .
$$

$$
\bar{M}=(X-\Sigma) \cup_{\theta}(L \times \Sigma \times[0,1)),
$$

with blowdown map $\beta: \bar{M} \rightarrow X$ given by the identity away from the boundary and by the quotient map from $L \times \Sigma \times[0,1)$ to $(L \times \Sigma \times[0,1)) /\left((z, y, 0) \sim\left(z^{\prime}, y, 0\right)\right)$ near the boundary. The blowup is a smooth manifold with boundary $Y=\partial \bar{M}=L \times \Sigma$. Let inc: $\partial \bar{M} \rightarrow \bar{M}$ be the inclusion of the boundary, and denote the projections onto 
the two components by $\pi_{L}: Y \rightarrow L$ and $\pi_{\Sigma}: Y \rightarrow \Sigma$. By an abuse of notation, we will also use $\pi_{L}$ and $\pi_{\Sigma}$ to denote the projections from $N-\Sigma \cong L \times \Sigma \times(0,1)$ to $L$ and $\Sigma$, respectively. Let $\pi_{Y}$ denote the projection from $N-\Sigma$ to $Y=L \times \Sigma$. The conifold transition of $X$, denoted by $\mathrm{CT}(X)$, is defined as

$$
\mathrm{CT}(X)=(X-\Sigma) \cup_{\theta}(L \times \Sigma \times[0,1)) /\left((z, y, 0) \sim\left(z, y^{\prime}, 0\right)\right) .
$$

The conifold transition is a stratified space with one singular stratum $L$, whose link is $\Sigma$. However, $\mathrm{CT}(X)$ is not always a pseudomanifold: If $X$ has one isolated singularity $\Sigma=\mathrm{pt}$, then $\mathrm{CT}(X)=\bar{M}$ is a manifold with boundary, the boundary constitutes the bottom stratum and the link is a point. Since the singular stratum does not have codimension at least two, this is not a pseudomanifold. If $\Sigma$ is positivedimensional, then $\mathrm{CT}(X)$ is a pseudomanifold. All of our theorems do apply even when $\operatorname{dim} \Sigma=0$. Let $\beta^{\prime}: \bar{M} \rightarrow \mathrm{CT}(X)$ be the blowdown map for the conifold transition of $X$, given by the quotient map. Note also the involutive character of this construction, $\mathrm{CT}(\mathrm{CT}(X)) \cong X$.

The coordinate $x$ in $(0,1)$ above may be extended to a smooth boundary-defining function on $\bar{M}$, that is, a nonnegative function, $x$, whose zero set is exactly $\partial \bar{M}$, and whose normal derivative does not vanish at $\partial \bar{M}$. We can now define the metrics we will consider on $M$, which may in fact be defined on a broader class of open manifolds.

Definition 2.1 Let $M$ be the interior of a manifold $\bar{M}$ with fibration boundary $\partial \bar{M} \cong Y \stackrel{\psi}{\rightarrow} \Sigma$ with fibre $L$ and boundary-defining function $x$. Assume that $Y$ can be covered by bundle charts $U_{i} \cong V_{i} \times L$ whose transition functions $f_{i j}$ have differentials $d f_{i j}$ that are diagonal with respect to some splitting $T Y \cong T L \oplus H$. A product-type fibred scattering metric on $M$ is a smooth metric that near $\partial \bar{M}$ has the form

$$
g_{\mathrm{fs}}=\frac{d x^{2}}{x^{4}}+\psi^{*} d s_{\Sigma}^{2}+\frac{h}{x^{2}}
$$

where $h$ is positive definite on $T L$ and vanishes on $H$.

Examples of such metrics are the natural Sasaki metrics [32] on the tangent bundle of a compact manifold, $\Sigma$. In this case, the boundary fibration of $M=T \Sigma$ is isomorphic to the spherical unit tangent bundle $S^{n} \rightarrow Y \rightarrow \Sigma$. We note that the condition on $Y$ in this definition is necessary for it to make sense. If the coordinate transition functions do not respect the splitting of $T Y$, then we cannot meaningfully scale in just the fibre direction. This is different from the four types of metrics below, which can be defined on any manifold with fibration boundary. 
A special subclass of these metrics arises when the boundary fibration is flat with respect to the structure group $\operatorname{Isom}(L)$ for some fixed metric $d s_{L}^{2}$ on $L$. In this case, we can require that the metric $g_{\text {fs }}$ be a product metric

$$
g_{\mathrm{fs}}=\frac{d x^{2}}{x^{4}}+d s_{V_{i}}^{2}+\frac{1}{x^{2}} d s_{L}^{2}
$$

on each chart $(0, \epsilon) \times U_{i} \cong(0, \epsilon) \times V_{i} \times L$ for the given fixed metric on $L$. In this case, we say that $g_{\mathrm{fs}}$ is a geometrically flat fibred scattering metric on $M$. This flatness condition arises also in the definition of $H I$ cohomology; see [4]. This of course can be arranged when the boundary fibration is a product, as in the case we consider in this paper.

Note that in the case that $\partial \bar{M}$ is a product $L \times \Sigma$, it carries two possible boundary fibrations: either $\psi: Y \rightarrow \Sigma$ or $\phi: Y \rightarrow L$. A fibred scattering metric on $M$ associated to the boundary fibration $\psi: \partial \bar{M} \rightarrow \Sigma$ is a fibred boundary metric on $M$ associated to the dual fibration $\phi: \partial \bar{M} \rightarrow L$. Fibred boundary metrics on $M$ associated to $\phi$ are conformal to a third class of metrics, called fibred cusp metrics. These two classes may be defined as follows:

- $g_{\mathrm{fb}}$ is called a (product-type) fibred boundary metric if near $\partial \bar{M}$ it takes the form

$$
g_{\mathrm{fb}}=\frac{d x^{2}}{x^{4}}+\frac{\phi^{*} d s_{L}^{2}}{x^{2}}+k,
$$

where $k$ is a symmetric two-tensor on $\partial \bar{M}$ which restricts to a metric on each fibre $\Sigma$ of $\phi: Y \rightarrow L$.

- $g_{\text {fc }}$ is called a (product-type) fibred cusp metric if near $\partial \bar{M}$ it takes the form

$$
g_{\mathrm{fc}}=\frac{d x^{2}}{x^{2}}+\phi^{*} d s_{L}^{2}+x^{2} k
$$

where $k$ is as above.

In the case that $\Sigma$ is a point, these two metrics reduce to the well-studied classes of b-metrics and cusp metrics, respectively (see eg [23] for more) and $g_{\text {fs }}$ becomes a scattering metric.

\section{Intersection spaces}

Intersection homology groups of a stratified space were introduced by Goresky and MacPherson in $[20 ; 21]$. In order to obtain independence of the stratification, they imposed on perversity functions $\bar{p}:\{2,3, \ldots\} \rightarrow\{0,1,2, \ldots\}$ the conditions $\bar{p}(2)=0$ and $\bar{p}(k) \leq \bar{p}(k+1) \leq \bar{p}(k)+1$. Theorems 1.2 and 1.3 , however, clearly involve 
perversities that do not satisfy these conditions. Thus in the present paper, a perversity $\bar{p}$ is just a sequence of integers $(\bar{p}(0), \bar{p}(1), \bar{p}(2), \ldots)$. (This is called an "extended" perversity; it is called a "loose" perversity in [26].)

Let $\bar{p}$ be an extended perversity. In [2], the first author introduced a homotopy-theoretic method that assigns to certain types of $n$-dimensional stratified pseudomanifolds $X$ CW-complexes

$$
I^{\bar{p}} X
$$

the perversity- $\bar{p}$ intersection spaces of $X$, such that for complementary perversities $\bar{p}$ and $\bar{q}$, there is a Poincaré duality isomorphism

$$
\tilde{H}^{i}\left(I^{\bar{p}} X\right) \cong \tilde{H}_{n-i}\left(I^{\bar{q}} X\right)
$$

when $X$ is compact and oriented, where $\widetilde{H}^{i}\left(I^{\bar{p}} X\right)$ denotes reduced singular cohomology of $I^{\bar{p}} X$ with real coefficients. If $\bar{p}=\bar{m}$ is the lower-middle perversity, we will briefly write $I X$ for $I^{\bar{m}} X$. The singular cohomology groups

$$
H I_{\bar{p}}^{*}(X)=H^{*}\left(I^{\bar{p}} X\right), \tilde{H} I_{\bar{p}}^{*}(X)=\widetilde{H}^{*}\left(I^{\bar{p}} X\right)
$$

define a new (unreduced/reduced) cohomology theory for stratified spaces, usually not isomorphic to intersection cohomology $I H_{\bar{p}}^{*}(X)$. This is already apparent from the observation that $H I_{\bar{p}}^{*}(X)$ is an algebra under cup product, whereas it is well known that $I H_{\bar{p}}^{*}(X)$ cannot generally, for every $\bar{p}$, be endowed with a $\bar{p}$-internal algebra structure. Let us put $H I^{*}(X)=H^{*}(I X)$.

Roughly speaking, the intersection space $I X$ associated to a singular space $X$ is defined by replacing links of singularities by their corresponding Moore approximations, ie spatial homology truncations. Let $L$ be a simply connected CW complex, and fix an integer $k$.

Definition 3.1 A stage- $k$ Moore approximation of $L$ is a CW complex $L_{<k}$, together with a structural map $f: L_{<k} \rightarrow L$, such that $f_{*}: H_{r}\left(L_{<k}\right) \rightarrow H_{r}(L)$ is an isomorphism if $r<k$, and $H_{r}\left(L_{<k}\right) \cong 0$ for all $r \geq k$.

Moore approximations exist for every $k$; see eg [2, Section 1.1]. If $k \leq 0$, then we take $L_{<k}=\varnothing$, the empty set. If $k=1$, we take $L_{<1}$ to be a point. The simple connectivity assumption is sufficient, but certainly not necessary. If $L$ is finite-dimensional and $k>\operatorname{dim} L$, then we take the structural map $f$ to be the identity. If every cellular $k$-chain is a cycle, then we can choose $L_{<k}=L^{(k-1)}$, the $(k-1)$-skeleton of $L$, with 
structural map given by the inclusion map, but in general, $f$ cannot be taken to be the inclusion of a subcomplex.

Let $X$ be an $n$-dimensional stratified pseudomanifold as in Section 2. Assume that the link $L$ of $\Sigma$ is simply connected. Let $l$ be the dimension of $L$. We shall recall the construction of associated perversity- $\bar{p}$ intersection spaces $I^{\bar{p}} X$ only for such $X$, though it is available in more generality; see eg [7]. Set $k=l-\bar{p}(l+1)$ and let $f: L_{<k} \rightarrow L$ be a stage- $k$ Moore approximation to $L$. Let $\bar{M}$ be the blowup of $X$ with boundary $\partial \bar{M}=Y=L \times \Sigma$. Let

$$
g: L_{<k} \times \Sigma \rightarrow \bar{M}
$$

be the composition

$$
L_{<k} \times \Sigma \stackrel{f \times \operatorname{id}_{\Sigma}}{\longrightarrow} L \times \Sigma=\partial \bar{M} \hookrightarrow \bar{M} .
$$

The intersection space is the homotopy cofibre of $g$ :

Definition 3.2 The perversity- $\bar{p}$ intersection space $I^{\bar{p}} X$ of $X$ is defined to be

$$
I^{\bar{p}} X=\operatorname{cone}(g)=\bar{M} \cup_{g} c\left(L_{<k} \times \Sigma\right) .
$$

Poincaré duality for this construction is Theorem 2.47 of [2]. For a topological space $Z$, let $Z^{+}$be the disjoint union of $Z$ with a point. Recall that the cone on the empty set is a point and hence $\operatorname{cone}(\varnothing \rightarrow Z)=Z^{+}$.

Proposition 3.3 Let $\bar{p}$ be an (extended) perversity and let $c$ be the codimension of the singular stratum $\Sigma$ in $X$. If $\bar{p}(c)<0$, then $\widetilde{H} I_{*}^{\bar{p}}(X) \cong H_{*}(\bar{M}, \partial \bar{M})$, and if $\bar{p}(c) \geq c-1$, then $\widetilde{H} I_{*}^{\bar{p}}(X) \cong H_{*}(\bar{M})$.

Proof If $\bar{p}(c)<0$, then $k>l$ and $L_{<k}=L$ with $f: L_{<k} \rightarrow L$ the identity. It follows that $I^{\bar{p}} X=\bar{M} \cup_{\partial \bar{M}} c(\partial \bar{M})$ and

$$
\widetilde{H} I_{*}^{\bar{p}}(X)=\widetilde{H}_{*}\left(\bar{M} \cup_{\partial \bar{M}} c(\partial \bar{M})\right) \cong H_{*}(\bar{M}, \partial \bar{M}) .
$$

If $\bar{p}(c) \geq c-1$, then $k \leq 0$, so $L_{<k}=\varnothing$. Thus, $I^{\bar{p}} X=\operatorname{cone}(\varnothing \rightarrow \bar{M})=\bar{M}^{+}$and

$$
\widetilde{H} I_{*}^{\bar{p}}(X)=\widetilde{H}_{*}\left(\bar{M}^{+}\right) \cong H_{*}(\bar{M}) \text {. }
$$

Example 3.4 Consider the equation

$$
y^{2}=x^{2}(x-1)
$$

or its homogeneous version $v^{2} w=u^{2}(u-w)$, defining a curve $X$ in $\mathbb{C P}^{2}$. A local 
isomorphism

$$
V=\left\{y^{2}=x^{2}(x-1)\right\} \rightarrow\left\{\eta^{2}=\xi^{2}\right\}
$$

near the origin is given by $\xi=x g(x)$ and $\eta=y$, with $g(x)=\sqrt{x-1}$ analytic and nonzero near 0 . The equation $\eta^{2}=\xi^{2}$ describes a nodal singularity at the origin in $\mathbb{C}^{2}$, whose link is $\partial I \times S^{1}$, two circles. All other points on the curve are nonsingular, as is easily seen from the gradient of the defining equation. It is homeomorphic to a pinched torus, that is, $T^{2}$ with a meridian collapsed to a point, or, equivalently, a cylinder $I \times S^{1}$ with coned-off boundary, where $I=[0,1]$. The ordinary homology group $H_{1}(X)$ has rank one and is generated by the longitudinal circle (while the meridian circle bounds the cone with vertex at the singular point of $X$ ). The intersection homology group $I H_{1}(X)$ agrees with the intersection homology of the normalization $S^{2}$ of $X$ (the longitude in $X$ is not an "allowed" 1-cycle, while the meridian bounds an allowed 2-chain), so

$$
I H_{1}(X)=I H_{1}\left(S^{2}\right)=H_{1}\left(S^{2}\right)=0 .
$$

The link of the singular point is $\partial I \times S^{1}$, two circles. The intersection space $I X$ of $X$ is a cylinder $I \times S^{1}$ together with an interval, whose one endpoint is attached to a point in $\{0\} \times S^{1}$ and whose other endpoint is attached to a point in $\{1\} \times S^{1}$. Thus $I X$ is homotopy equivalent to the figure eight and

$$
H_{1}(I X)=\mathbb{R} \oplus \mathbb{R} .
$$

Remark 3.5 As suggested by the previous example, the middle homology of the intersection space $I X$ usually takes into account more cycles than the corresponding intersection homology group of $X$. More precisely, for $X^{2 k}$ with only isolated singularities $\Sigma$, the group $I H_{k}(X)$ is generally smaller than both $H_{k}(X-\Sigma)$ and $H_{k}(X)$, being a quotient of the former and a subgroup of the latter, while $H_{k}(I X)$ is generally bigger than both $H_{k}(X-\Sigma)$ and $H_{k}(X)$, containing the former as a subgroup and mapping to the latter surjectively; see [2].

One advantage of the intersection space approach is a richer algebraic structure: The Goresky-MacPherson intersection cochain complexes $I C_{\bar{p}}^{*}(X)$ are generally not algebras, unless $\bar{p}$ is the zero-perversity, in which case $I C_{\bar{p}}^{*}(X)$ is essentially the ordinary cochain complex of $X$. (The Goresky-MacPherson intersection product raises perversities in general.) Similarly, Cheeger's differential complex $\Omega_{(2)}^{*}(X)$ of $L^{2}$ forms on the top stratum with respect to his conical metric is not an algebra under wedge product of forms. Using the intersection space framework, the ordinary cochain complex $C^{*}\left(I^{\bar{p}} X\right)$ of $I^{\bar{p}} X$ is a DGA, simply by employing the ordinary cup product. 
Another advantage of introducing intersection spaces is the possibility of discussing the intersection $K$-theory $K^{*}\left(I^{\bar{p}} X\right)$, which is not possible using intersection chains, since nontrivial generalized cohomology theories such as $K$-theory do not factor through cochain theories.

\section{Intersection homology}

We need to use a version of intersection homology that behaves correctly for extended perversities. More precisely, the singular intersection homology of [26], which agrees with the Goresky-MacPherson intersection homology, displays the following anomaly for very large perversity values: If $A$ is a closed (n-1)-dimensional manifold and $c^{\circ} A$ the open cone on $A$, then the intersection homology of $c^{\circ} A$ vanishes in degrees greater than or equal to $n-1-\bar{p}(n)$, with one exception, namely, if the degree is 0 and $0 \geq n-1-\bar{p}(n)$, then the intersection homology of the cone is $\mathbb{Z}$. Now if the perversity $\bar{p}$ satisfies the Goresky-MacPherson growth conditions, then this exception can never arise, since $\bar{p}(n) \leq n-2$. But if $\bar{p}$ is arbitrary, the exception may very well occur.

To correct this anomaly, we use the modification of Saralegi [31] and, independently, Friedman [17]. Let $\Delta_{i}$ denote the standard $i$-simplex and let $\Delta_{i}^{j} \subset \Delta_{i}$ be the $j-$ skeleton of $\Delta_{i}$. Let $X$ be any stratified space with singular set $\Sigma$ and $\bar{p}$ be an arbitrary (extended) perversity. Let $C_{*}(X)=C_{*}(X ; \mathbb{R})$ denote the singular chain complex with $\mathbb{R}$-coefficients of $X$. A singular $i$-simplex $\sigma: \Delta_{i} \rightarrow X$ is called $\bar{p}$-allowable if for every pure stratum $S$ of $X$,

$$
\sigma^{-1}(S) \subset \Delta_{i}^{i-k+\bar{p}(k)} \text {, where } k=\operatorname{codim} S .
$$

(This definition is due to King [26].) For each $i=0,1,2, \ldots$, let $C_{i}^{\bar{p}}(X) \subset C_{i}(X)$ be the linear subspace generated by the $\bar{p}$-allowable singular $i$-simplices whose image is not entirely contained in $\Sigma$. If $\xi \in C_{i}^{\bar{p}}(X)$, then its chain boundary $\partial \xi \in C_{i-1}(X)$ can be uniquely written as $\partial \xi=\beta_{\Sigma}+\beta$, where $\beta_{\Sigma}$ is a linear combination of singular simplices whose image lies entirely in $\Sigma$, whereas $\beta$ is a linear combination of simplices each of which touches at least one point of $X-\Sigma$. We set $\partial^{\prime} \xi=\beta$ and

$$
I C_{i}^{\bar{p}}(X)=\left\{\xi \in C_{i}^{\bar{p}}(X) \mid \partial^{\prime} \xi \in C_{i-1}^{\bar{p}}(X)\right\} .
$$

It is readily verified that $\partial^{\prime}$ is linear and $\left(I C_{*}^{\bar{p}}(X), \partial^{\prime}\right)$ is a chain complex. The version of intersection homology that we shall use in this paper is then given by

$$
I H_{i}^{\bar{p}}(X)=H_{i}\left(I C_{*}^{\bar{p}}(X)\right) .
$$


Friedman [17] shows that if $\bar{p}(k) \leq k-2$ for all $k$, then $I H_{*}^{\bar{p}}(X)$ as defined here agrees with the definition of singular intersection homology as given by Goresky, MacPherson and King. If $A$ is a closed $a$-dimensional manifold, then

$$
I H_{i}^{\bar{p}}\left(c^{\circ} A\right) \cong\left\{\begin{array}{cl}
0 & \text { if } i \geq a-\bar{p}(a+1) \\
I H_{i}^{\bar{p}}(A) & \text { if } i<a-\bar{p}(a+1)
\end{array}\right.
$$

and this holds even for degree $i=0$, ie the above anomaly has been corrected. If $A$ is unstratified (that is, has only one stratum, the regular stratum), then $I H_{i}^{\bar{p}}(A)=H_{i}(A)$. If $A$ is however not intrinsically stratified, then one can in general not compute $I H_{*}^{\bar{p}}(A)$ by ordinary homology, since $I H_{*}^{\bar{p}}$ is not a topological invariant anymore for arbitrary perversities $\bar{p}$.

According to [17], $I H_{*}^{\bar{p}}$ has Mayer-Vietoris sequences: If $U, V \subset X$ are open such that $X=U \cup V$, then there is an exact sequence

$$
\cdots \rightarrow I H_{i}^{\bar{p}}(U \cap V) \rightarrow I H_{i}^{\bar{p}}(U) \oplus I H_{i}^{\bar{p}}(V) \rightarrow I H_{i}^{\bar{p}}(X) \rightarrow I H_{i-1}^{\bar{p}}(U \cap V) \rightarrow \cdots
$$

Furthermore, if $M$ is any (unstratified) manifold (not necessarily compact) and $X$ a stratified space, then the Künneth formula

$$
I H_{*}^{\bar{p}}(M \times X) \cong H_{*}(M) \otimes I H_{*}^{\bar{p}}(X)
$$

holds if the strata of $M \times X$ are the products of $M$ with the strata of $X$. (When $\bar{p}(k) \leq k-2$ for all $k$, this is Theorem 4 with $\mathbb{R}$-coefficients in [26].)

Proposition 4.1 Let $\bar{q}$ be an (extended) perversity and let $c$ be the codimension of the singular stratum $L$ in the conifold transition $\mathrm{CT}(X)$. If $\bar{q}(c)<0$, then $I H_{*}^{\bar{q}}(\mathrm{CT}(X)) \cong$ $H_{*}(\bar{M})$, and if $\bar{q}(c) \geq c-1$, then $I H_{*}^{\bar{q}}(\mathrm{CT}(X)) \cong H_{*}(\bar{M}, \partial \bar{M})$.

Proof Set $I_{j}=I H_{j}^{\bar{q}}\left(L \times c^{\circ} \Sigma\right)$ and let $s=c-1$ be the dimension of $\Sigma$. Then by (1) and (3),

$$
\begin{aligned}
I_{*} & \cong H_{*}(L) \otimes I H_{*}^{\bar{q}}\left(c^{\circ} \Sigma\right) \\
& =H_{*}(L) \otimes \tau_{\leq s-1-\bar{q}(s+1)} H_{*}(\Sigma)=H_{*}(L) \otimes \tau_{\leq c-2-\bar{q}(c)} H_{*}(\Sigma) .
\end{aligned}
$$

If $\bar{q}(c) \geq c-1$, that is, $c-2-\bar{q}(c)<0$, then $I_{*}=0$, so $I H_{*}^{\bar{q}}(\mathrm{CT}(X)) \cong H_{*}(\bar{M}, \partial \bar{M})$ by the Mayer-Vietoris sequence of the open cover $\mathrm{CT}(X)=M \cup\left(L \times c^{\circ} \Sigma\right)$ and the five lemma. If $\bar{q}(c)<0$, that is, $c-2-\bar{q}(c) \geq s$, then $I_{*}=H_{*}(L \times \Sigma)$, so $I H_{*}^{\bar{q}}(\mathrm{CT}(X)) \cong H_{*}(\bar{M})$, again by a Mayer-Vietoris argument. 


\section{Proof of Theorem 1.2}

Let $j$ be any nonnegative degree. Throughout this entire section, $j$ will remain fixed and we must establish an isomorphism $\tilde{H} I_{j}^{\bar{p}}(X) \cong I G_{j}^{(n-1-\bar{p}(l+1)-j)}(\mathrm{CT}(X))$, where $l=\operatorname{dim} L$. Let $\bar{M}$ be the blowup of $X$ and $M$ its interior. Let $c$ be the codimension of the singular set $L$ in $\mathrm{CT}(X)$ and $\hat{c}$ be the codimension of the singular set $\Sigma$ in $X$, that is,

$$
c=n-l \quad \text { and } \quad \hat{c}=l+1 .
$$

Set $k=\widehat{c}-1-\bar{p}(\widehat{c})$ and let $f: L_{<k} \rightarrow L$ be a stage- $k$ Moore approximation of $L$. Then the intersection space $I^{\bar{p}} X$ is the mapping cone $I^{\bar{p}} X=\operatorname{cone}(g)$ of the map $g: L_{<k} \times \Sigma \rightarrow \bar{M}$ given by the composition

$$
L_{<k} \times \Sigma \stackrel{f \times \mathrm{id}_{\Sigma}}{\longrightarrow} L \times \Sigma=\partial \bar{M} \hookrightarrow \bar{M} .
$$

Let

$$
\gamma: I H_{j}^{\bar{q}}(\mathrm{CT}(X)) \rightarrow I H_{j}^{\bar{q}^{\prime}}(\mathrm{CT}(X))
$$

be the canonical map, where $\bar{q}(c)=n-2-\bar{p}(l+1)-j$ and $\bar{q}^{\prime}(c)=\bar{q}(c)+1$. Note that the perversities $\bar{q}$ and $\bar{q}^{\prime}$ depend on the degree $j$. Then by definition

$$
I G_{j}^{(n-1-\bar{p}(l+1)-j)}(\mathrm{CT}(X))=I H_{j}^{\bar{q}}(\mathrm{CT}(X)) \oplus \operatorname{coker}(\gamma) .
$$

The strategy of the proof is to compute intersection homology and $H I$ near the singular stratum using Künneth theorems ("local calculations"), then determine maps ("local maps") between these groups near the singularities, and finally to assemble this information to global information using Mayer-Vietoris techniques. Our arguments do not extend to integer coefficients; field coefficients are essential.

We begin with the local calculations. Let $B_{*}=H_{*}(L \times \Sigma)$ be the homology of the boundary, $T_{*}=H_{*}(M)$ the homology of the top stratum, $I_{*}=I H_{*}^{\bar{q}}\left(L \times c^{\circ} \Sigma\right)$, $J_{*}=I H_{*}^{\bar{q}^{\prime}}\left(L \times c^{\circ} \Sigma\right)$ and $R_{*}=H_{*}\left(\operatorname{cone}\left(f \times \mathrm{id}_{\Sigma}\right)\right)$. Again, we stress that the graded vector spaces $I_{*}$ and $J_{*}$ depend on the degree $j$.

Lemma 5.1 The canonical inclusion $L \rightarrow \operatorname{cone}(f)$ induces an isomorphism

$$
\tau_{\geq k} H_{*}(L) \cong \widetilde{H}_{*}(\operatorname{cone}(f)) .
$$

Proof The reduced homology of the mapping cone of $f$ fits into an exact sequence $\cdots \rightarrow H_{i}\left(L_{<k}\right) \stackrel{f_{*}}{\longrightarrow} H_{i}(L) \rightarrow \tilde{H}_{i}(\operatorname{cone}(f)) \rightarrow H_{i-1}\left(L_{<k}\right) \stackrel{f_{*}}{\longrightarrow} H_{i-1}(L) \rightarrow \cdots$. 
We distinguish the three cases $i=k, i>k$ and $i<k$. If $i<k$, then $f_{*}$ on $H_{i}\left(L_{<k}\right)$ and on $H_{i-1}\left(L_{<k}\right)$ is an isomorphism and thus $\tilde{H}_{i}$ (cone $\left.(f)\right)=0$. If $i=k$, then $H_{i}\left(L_{<k}\right)=0$ and $f_{*}$ on $H_{i-1}\left(L_{<k}\right)$ is an isomorphism. Therefore, $H_{i}(L) \rightarrow$ $\widetilde{H}_{i}$ (cone $\left.(f)\right)$ is an isomorphism. Finally, if $i>k$, then both $H_{i}\left(L_{<k}\right)$ and $H_{i-1}\left(L_{<k}\right)$ vanish and again $H_{i}(L) \rightarrow \tilde{H}_{i}(\operatorname{cone}(f))$ is an isomorphism.

Remark 5.2 If $k \leq 0$, then $L_{<k}=\varnothing$ and $\operatorname{cone}(f)=L^{+}$. It follows that in degree 0 ,

$$
\left(\tau_{\geq k} H_{*}(L)\right)_{0}=H_{0}(L) \cong \tilde{H}_{0}\left(L^{+}\right)=\tilde{H}_{0}(\operatorname{cone}(f)),
$$

in accordance with the lemma.

Let $v \in \operatorname{cone}(f)$ be the cone vertex and let $Q=(\operatorname{cone}(f) \times \Sigma) /(\{v\} \times \Sigma)$, which is homeomorphic to cone $\left(f \times \operatorname{id}_{\Sigma}\right)$. As the inclusion $\{v\} \times \Sigma \rightarrow \operatorname{cone}(f) \times \Sigma$ is a closed cofibration, the quotient map induces an isomorphism

$H_{*}((\operatorname{cone}(f),\{v\}) \times \Sigma)=H_{*}(\operatorname{cone}(f) \times \Sigma,\{v\} \times \Sigma) \cong \tilde{H}_{*}(Q) \cong \tilde{H}_{*}\left(\operatorname{cone}\left(f \times \operatorname{id}_{\Sigma}\right)\right)$.

By the Künneth theorem for relative homology,

$$
H_{*}((\operatorname{cone}(f),\{v\}) \times \Sigma) \cong H_{*}(\operatorname{cone}(f),\{v\}) \otimes H_{*}(\Sigma)=\tilde{H}_{*}(\operatorname{cone}(f)) \otimes H_{*}(\Sigma) .
$$

Composing, we obtain an isomorphism

$$
\widetilde{H}_{*}\left(\operatorname{cone}\left(f \times \operatorname{id}_{\Sigma}\right)\right) \cong \widetilde{H}_{*}(\operatorname{cone}(f)) \otimes H_{*}(\Sigma) .
$$

Composing with the isomorphism of Lemma 5.1, we get an isomorphism

$$
\tilde{H}_{*}\left(\operatorname{cone}\left(f \times \operatorname{id}_{\Sigma}\right)\right) \cong\left(\tau_{\geq k} H_{*}(L)\right) \otimes H_{*}(\Sigma) .
$$

Remark 5.3 If $k \leq 0$, then $L_{<k} \times \Sigma=\varnothing$ and thus cone $\left(f \times \operatorname{id}_{\Sigma}\right)=(L \times \Sigma)^{+}$. This is consistent with

$$
\operatorname{cone}\left(f \times \operatorname{id}_{\Sigma}\right) \cong Q=\frac{\operatorname{cone}(f) \times \Sigma}{\{v\} \times \Sigma}=\frac{L^{+} \times \Sigma}{\{v\} \times \Sigma}=\frac{\{v\} \times \Sigma \sqcup L \times \Sigma}{\{v\} \times \Sigma}=(L \times \Sigma)^{+} .
$$

It will be convenient to put $a=\bar{p}(l+1)+j-l$; then the relation

$$
a+k=j
$$

holds. We compute the terms $R_{*}$ : 
Lemma 5.4 If $j>0$, then the isomorphism (4) induces an isomorphism

$$
R_{j} \cong \bigoplus_{t=0}^{a} H_{j-t}(L) \otimes H_{t}(\Sigma)
$$

(If $a<0$, this reads $R_{j}=0$.) Furthermore,

$$
R_{0} \cong\left\{\begin{array}{cc}
\mathbb{R} & \text { if } k>0, \\
\mathbb{R} \oplus\left(H_{0}(L) \otimes H_{0}(\Sigma)\right) & \text { if } k \leq 0 .
\end{array}\right.
$$

Proof We start by observing that $k$ is independent of $j$. Now for $j>0$, reduced and unreduced homology coincide, so

$$
R_{j}=\tilde{H}_{j}\left(\operatorname{cone}\left(f \times \operatorname{id}_{\Sigma}\right)\right) \cong \bigoplus_{t=0}^{j}\left(\tau_{\geq k} H_{*}(L)\right)_{j-t} \otimes H_{t}(\Sigma) .
$$

Using (5), $j-t \geq k$ if and only if $a=j-k \geq t$. Thus

$$
R_{j} \cong \bigoplus_{t=0}^{a} H_{j-t}(L) \otimes H_{t}(\Sigma),
$$

since if $a>j$ and $j<t \leq a$, then $j-t<0$, so that $H_{j-t}(L)=0$.
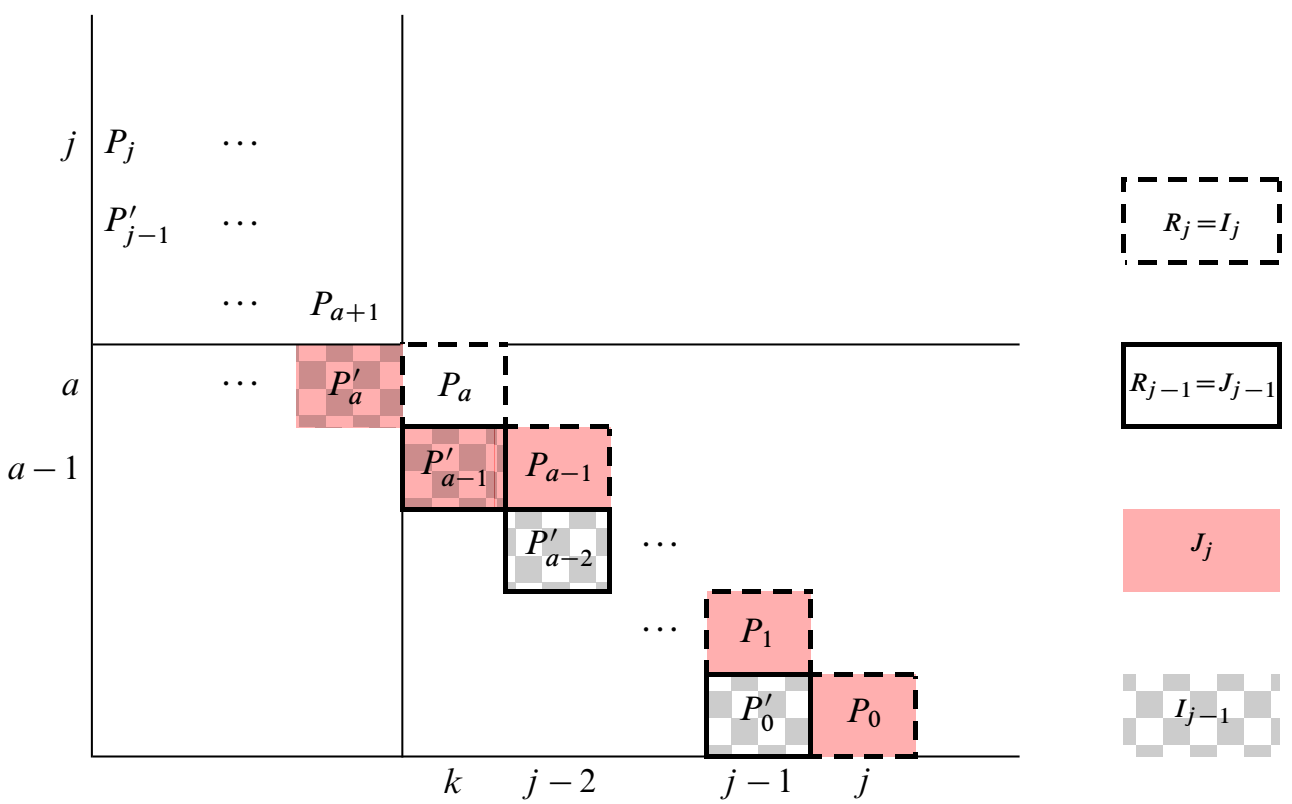

Figure 1: Local Künneth factor truncations when $j \neq 0$ 
In degree 0 , we find

$$
R_{0}=\mathbb{R} \oplus \tilde{H}_{0}\left(\operatorname{cone}\left(f \times \operatorname{id}_{\Sigma}\right)\right) \cong \mathbb{R} \oplus\left(\left(\tau_{\geq k} H_{*}(L)\right)_{0} \otimes H_{0}(\Sigma)\right)
$$

and $\left(\tau_{\geq k} H_{*}(L)\right)_{0}=0$ for $k>0$, whereas $\left(\tau_{\geq k} H_{*}(L)\right)_{0}=H_{0}(L)$ for $k \leq 0$.

With $s=\operatorname{dim} \Sigma$, we have $s+1=n-l=c$ and thus according to (1),

$$
I H_{*}^{\bar{q}}\left(c^{\circ} \Sigma\right) \cong \tau_{<s-\bar{q}(s+1)} H_{*}(\Sigma)=\tau_{\leq a} H_{*}(\Sigma)
$$

for

$$
s-1-\bar{q}(s+1)=s-1-\bar{q}(c)=n-l-2-(n-2-\bar{p}(l+1)-j)=a .
$$

Consequently by the Künneth formula (3) for intersection homology,

$$
\begin{aligned}
I_{j} & \cong\left(H_{*}(L) \otimes I H_{*}^{\bar{q}}\left(c^{\circ} \Sigma\right)\right)_{j} \\
& \cong\left(H_{*}(L) \otimes \tau_{\leq a} H_{*}(\Sigma)\right)_{j}=\bigoplus_{t=0}^{a} H_{j-t}(L) \otimes H_{t}(\Sigma), \\
I_{j-1} & \cong\left(H_{*}(L) \otimes I H_{*}^{\bar{q}}\left(c^{\circ} \Sigma\right)\right)_{j-1} \\
& \cong\left(H_{*}(L) \otimes \tau_{\leq a} H_{*}(\Sigma)\right)_{j-1}=\bigoplus_{t=0}^{a} H_{j-1-t}(L) \otimes H_{t}(\Sigma) .
\end{aligned}
$$

Similarly for $\bar{q}^{\prime}$,

$$
\begin{aligned}
& J_{j} \cong\left(H_{*}(L) \otimes I H_{*}^{\bar{q}^{\prime}}\left(c^{\circ} \Sigma\right)\right)_{j} \\
& \cong\left(H_{*}(L) \otimes \tau_{\leq a-1} H_{*}(\Sigma)\right)_{j}=\bigoplus_{t=0}^{a-1} H_{j-t}(L) \otimes H_{t}(\Sigma), \\
& J_{j-1} \cong\left(H_{*}(L) \otimes I H_{*}^{\bar{q}^{\prime}}\left(c^{\circ} \Sigma\right)\right)_{j-1} \cong \bigoplus_{t=0}^{a-1} H_{j-1-t}(L) \otimes H_{t}(\Sigma) .
\end{aligned}
$$

This concludes the local calculations of groups. We can get a bit of help understanding how these pieces fit together, at least when $j \neq 0$, by setting $P_{t}=H_{j-t}(L) \otimes H_{t}(\Sigma)$ and letting $[j, a]: \bigoplus_{t=0}^{j} P_{t} \rightarrow \bigoplus_{t=0}^{a} P_{t}$ be the standard projection if $j>a$ and the identity if $j \leq a$. (Recall that when $j<a$ and $j<t \leq a$, then $P_{t}=0$ since then $j-t<0$ and $H_{j-t}(L)=0$.) Analogously, let $P_{t}^{\prime}=H_{j-1-t}(L) \otimes H_{t}(\Sigma)$ and define $[j-1, a]: \bigoplus_{t=0}^{j-1} P_{t}^{\prime} \rightarrow \bigoplus_{t=0}^{a} P_{t}^{\prime}$ to be the standard projection if $j-1>a$ and the identity if $j-1 \leq a$.

We picture how these pieces fit together in Figure 1. 
We commence the determination of various local maps near the singularities. Let $\gamma_{i}^{\text {loc }:} I_{i} \rightarrow J_{i}$ the canonical map. Using the collar associated to the boundary of the blowup, the open inclusion $L \times \Sigma \times(0,1) \hookrightarrow M$ induces a map

$$
\beta^{T}: B_{*} \rightarrow T_{*},
$$

while the open inclusion $L \times \Sigma \times(0,1) \hookrightarrow L \times c^{\circ} \Sigma$ induces maps

$$
\beta^{I}: B_{*} \rightarrow I_{*} \quad \text { and } \quad \beta^{J}: B_{*} \rightarrow J_{*}
$$

such that

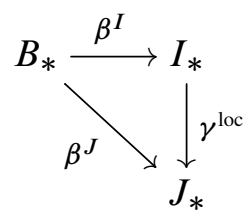

commutes. The canonical inclusion $L \times \Sigma \hookrightarrow \operatorname{cone}\left(f \times \mathrm{id}_{\Sigma}\right)$ induces a map

$$
\beta^{R}: B_{*} \rightarrow R_{*} .
$$

The diagram

$$
\begin{gathered}
B_{j} \stackrel{\beta_{j}^{I}}{\times \uparrow \cong} I_{j} \\
\bigoplus_{t=0}^{j} P_{t} \underset{[j, a]}{\longrightarrow} \bigoplus_{t=0}^{a} P_{t}
\end{gathered}
$$

commutes. The vertical isomorphisms are given by the cross product. For $j>0$, let $\varphi^{\text {loc }}: I_{j} \rightarrow R_{j}$ be the unique isomorphism such that

$$
\begin{gathered}
I_{j}--{\frac{\varphi^{\mathrm{loc}}}{-}-}^{\rightarrow} R_{j} \\
\times \uparrow \cong \cong \times \\
\bigoplus_{t=0}^{a} P_{t} \cong \bigoplus_{t=0}^{a} P_{t}
\end{gathered}
$$

commutes, using Lemma 5.4. Since

$$
\begin{array}{cc}
B_{j} \longrightarrow \beta_{j}^{R} & R_{j} \\
\times \uparrow \cong & \cong \uparrow \times \\
\bigoplus_{t=0}^{j} P_{t} \underset{[j, a]}{\longrightarrow} \bigoplus_{t=0}^{a} P_{t}
\end{array}
$$


commutes, we know that

$$
\begin{aligned}
B_{j} \stackrel{\beta_{j}^{I}}{\longrightarrow} & I_{j} \\
\beta_{j}^{R} \searrow \underset{\downarrow^{\mathrm{loc}}}{R_{j}} &
\end{aligned}
$$

commutes also. For $j>1$, let $\psi^{\text {loc }}: I_{j-1} \rightarrow R_{j-1}$ be the unique epimorphism such that

$$
\begin{array}{cc}
I_{j-1}--\psi^{\text {loc }}-\# & R_{j-1} \\
\times \uparrow \cong \quad \cong \uparrow \times \\
\bigoplus_{t=0}^{a} P_{t}^{\prime} \stackrel{[a, a-1]}{\longrightarrow} \bigoplus_{t=0}^{a-1} P_{t}^{\prime}
\end{array}
$$

commutes, using Lemma 5.4. Under the cross product, the commutative diagram

$$
\begin{aligned}
& \bigoplus_{t=0}^{j-1} P_{t}^{\prime} \stackrel{[j-1, a]}{\longrightarrow} \bigoplus_{t=0}^{a} P_{t}^{\prime} \\
& \underset{[j-1, a-1]}{\Downarrow} \downarrow[a, a-1] \\
& \bigoplus_{t=0}^{a-1} P_{t}^{\prime}
\end{aligned}
$$

corresponds to

$$
B_{j-1} \stackrel{\beta_{j-1}^{I}}{\longrightarrow} I_{j-1}
$$

and therefore this also commutes.

For any $j$, the diagrams

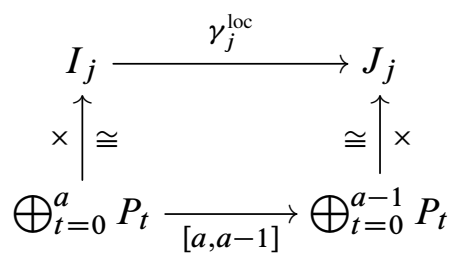

and

$$
\begin{array}{cc}
I_{j-1} \stackrel{\gamma_{j-1}^{\mathrm{loc}}}{\longrightarrow} J_{j-1} \\
\times \uparrow \cong \uparrow \times \\
\bigoplus_{t=0}^{a} P_{t}^{\prime} \underset{[a, a-1]}{\longrightarrow} \bigoplus_{t=0}^{a-1} P_{t}^{\prime}
\end{array}
$$


commute, showing that both $\gamma_{j}^{\text {loc }}$ and $\gamma_{j-1}^{\text {loc }}$ are surjective. For $j>1$, let $R_{j-1} \rightarrow J_{j-1}$ be the unique isomorphism such that

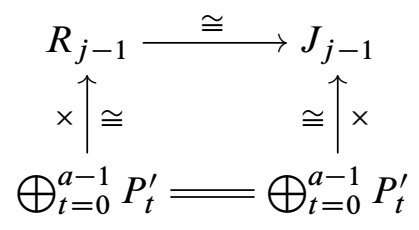

commutes. Then, since $\psi^{\text {loc }}$ and $\gamma_{j-1}^{\text {loc }}$ are both under the Künneth isomorphism given by the projection $[a, a-1]$, the diagram

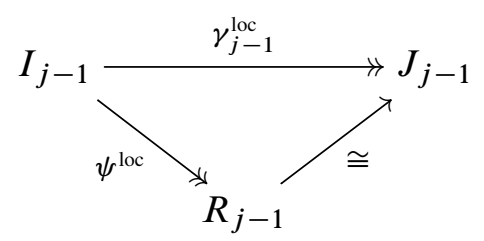

commutes $(j>1)$.

Lemma 5.5 When $j>1$, the identity $\operatorname{ker} \beta_{j-1}^{J}=\operatorname{ker} \beta_{j-1}^{R}$ holds in $B_{j-1}$.

Proof By diagram (9), in the case $j>1$ there is an isomorphism $v: R_{j-1} \rightarrow J_{j-1}$ such that $\nu \psi^{\text {loc }}=\gamma_{j-1}^{\text {loc }}$. According to diagram (8), $\beta_{j-1}^{R}=\psi^{\text {loc }} \beta_{j-1}^{I}$. Furthermore, $\beta_{j-1}^{J}=\gamma_{j-1}^{\text {loc }} \beta_{j-1}^{I}$ by diagram (6). Hence $\beta_{j-1}^{R}(x)=\psi^{\text {loc }} \beta_{j-1}^{I}(x)$ vanishes if and only if

$$
\nu \psi^{\mathrm{loc}} \beta_{j-1}^{I}(x)=\gamma_{j-1}^{\mathrm{loc}} \beta_{j-1}^{I}(x)=\beta_{j-1}^{J}(x)
$$

vanishes.

This concludes our investigation of local maps.

We move on to global arguments. The open cover $\mathrm{CT}(X)=M \cup\left(L \times c^{\circ} \Sigma\right)$ with $M \cap\left(L \times c^{\circ} \Sigma\right)=L \times \Sigma \times(0,1) \simeq L \times \Sigma$ yields a Mayer-Vietoris sequence for intersection homology

$$
B_{j} \stackrel{\beta_{j}}{\longrightarrow} T_{j} \oplus I_{j} \stackrel{\theta}{\longrightarrow} I H_{j}^{\bar{q}}(\mathrm{CT}(X)) \stackrel{\partial_{*}}{\longrightarrow} B_{j-1} \stackrel{\beta_{j-1}}{\longrightarrow} T_{j-1} \oplus I_{j-1} ;
$$

see (2). Similarly, there is such a sequence for perversity $\bar{q}^{\prime}$ :

$$
B_{j} \stackrel{\beta_{j}^{\prime}}{\longrightarrow} T_{j} \oplus J_{j} \stackrel{\theta^{\prime}}{\longrightarrow} I H_{j}^{\bar{q}^{\prime}}(\mathrm{CT}(X)) \stackrel{\partial_{*}}{\longrightarrow} B_{j-1} \stackrel{\beta_{j-1}^{\prime}}{\longrightarrow} T_{j-1} \oplus J_{j-1} .
$$


The canonical map from perversity $\bar{q}$ to $\bar{q}^{\prime}$ induces a commutative diagram

$$
\begin{aligned}
& B_{j} \stackrel{\beta_{j}}{\longrightarrow} T_{j} \oplus I_{j} \stackrel{\theta}{\longrightarrow} I H_{j}^{\bar{q}}(\mathrm{CT}(X)) \stackrel{\partial_{*}}{\longrightarrow} B_{j-1} \stackrel{\beta_{j-1}}{\longrightarrow} T_{j-1} \oplus I_{j-1}
\end{aligned}
$$

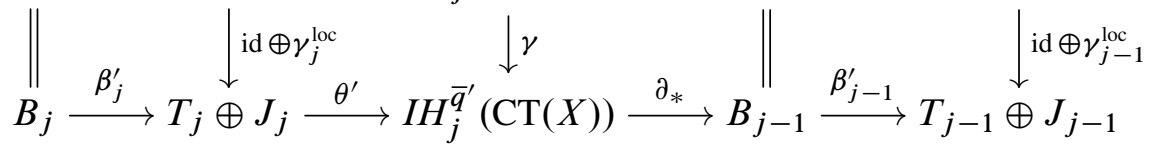

The subset

$$
U=((0,1] \times L \times \Sigma) \cup_{\{1\} \times L \times \Sigma} \operatorname{cone}\left(f \times \operatorname{id}_{\Sigma}\right) \simeq \operatorname{cone}\left(f \times \operatorname{id}_{\Sigma}\right)
$$

is open in the intersection space $I^{\bar{p}} X$. The open cover $I^{\bar{p}} X=M \cup U$ with $M \cap U=$ $L \times \Sigma \times(0,1) \simeq L \times \Sigma$ yields a Mayer-Vietoris sequence

$$
B_{j} \stackrel{\beta_{j}^{\prime \prime}}{\longrightarrow} T_{j} \oplus R_{j} \stackrel{\theta^{\prime \prime}}{\longrightarrow} H I_{j}^{\bar{p}}(X) \stackrel{\partial_{*}}{\longrightarrow} B_{j-1} \stackrel{\beta_{j-1}^{\prime \prime}}{\longrightarrow} T_{j-1} \oplus R_{j-1},
$$

the standard Mayer-Vietoris sequence for singular homology of topological spaces.

We shall prove Theorem 1.2 first for all $j>1$. Using the commutative diagrams (7) and (8), we obtain the following commutative diagram with exact rows:

$$
\begin{aligned}
& B_{j} \stackrel{\beta_{j}}{\longrightarrow} T_{j} \oplus I_{j} \stackrel{\theta}{\longrightarrow} I H_{j}^{\bar{q}}(\mathrm{CT}(X)) \stackrel{\partial_{*}}{\longrightarrow} B_{j-1} \stackrel{\beta_{j-1}}{\longrightarrow} T_{j-1} \oplus I_{j-1}
\end{aligned}
$$

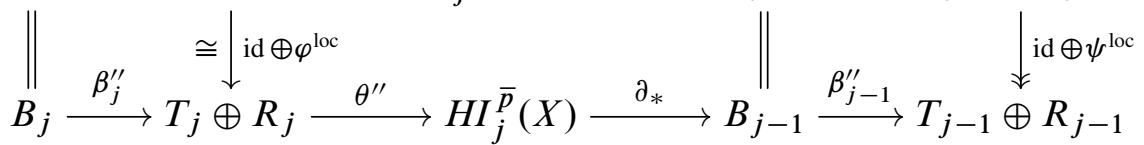

There exists a (nonunique) map $\rho: I H_{j}^{\bar{q}}(\mathrm{CT}(X)) \rightarrow H I_{j}^{\bar{p}}(X)$ which fills in diagram (11) commutatively; see eg [2, Lemma 2.46]. By the four lemma, $\rho$ is a monomorphism. This shows that $H I_{j}^{\bar{p}}(X)$ contains $I H_{j}^{\bar{q}}(\mathrm{CT}(X))$ as a subspace. Hence the theorem will follow from:

Proposition 5.6 If $j>1$, there is an isomorphism $\operatorname{coker} \rho \cong \operatorname{coker} \gamma$.

Proof Let us determine the cokernel of $\gamma$. Let $\gamma_{B}$ be the restriction of id: $B_{j-1} \rightarrow B_{j-1}$ to $\gamma_{B}: \operatorname{ker} \beta_{j-1} \rightarrow \operatorname{ker} \beta_{j-1}^{\prime}$, that is, $\gamma_{B}$ is the inclusion $\operatorname{ker} \beta_{j-1} \subset \operatorname{ker} \beta_{j-1}^{\prime}$. Let $\gamma_{\theta}: \operatorname{im} \theta \rightarrow \operatorname{im} \theta^{\prime}$ be obtained by restricting $\gamma$. Applying the snake lemma to the commutative diagram

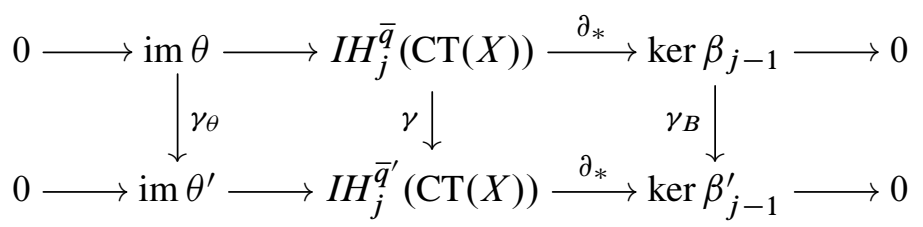


yields an exact sequence

$$
0 \rightarrow \operatorname{ker} \gamma_{\theta} \rightarrow \operatorname{ker} \gamma \rightarrow \operatorname{ker} \gamma_{B} \rightarrow \operatorname{coker} \gamma_{\theta} \rightarrow \operatorname{coker} \gamma \rightarrow \operatorname{coker} \gamma_{B} \rightarrow 0
$$

Since ker $\gamma_{B}=0$, we can extract the short exact sequence

$$
0 \rightarrow \operatorname{coker} \gamma_{\theta} \rightarrow \operatorname{coker} \gamma \rightarrow \operatorname{coker} \gamma_{B} \rightarrow 0
$$

As $\gamma_{j}^{\text {loc }}$ is surjective, the diagram

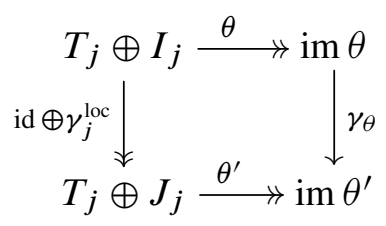

shows that $\gamma_{\theta}$ is also surjective and thus $\operatorname{coker} \gamma_{\theta}=0$. Therefore, we obtain an isomorphism

$$
\operatorname{coker} \gamma \stackrel{\cong}{\longrightarrow} \operatorname{coker} \gamma_{B}=\frac{\operatorname{ker} \beta_{j-1}^{\prime}}{\operatorname{ker} \beta_{j-1}}=\frac{\operatorname{ker} \beta_{j-1}^{T} \cap \operatorname{ker} \beta_{j-1}^{J}}{\operatorname{ker} \beta_{j-1}^{T} \cap \operatorname{ker} \beta_{j-1}^{I}},
$$

since $\beta_{j-1}=\left(\beta_{j-1}^{T}, \beta_{j-1}^{I}\right)$ and $\beta_{j-1}^{\prime}=\left(\beta_{j-1}^{T}, \beta_{j-1}^{J}\right)$.

Similarly, we determine the cokernel of $\rho$. Let $\rho_{B}$ be the restriction of id: $B_{j-1} \rightarrow B_{j-1}$ to $\rho_{B}: \operatorname{ker} \beta_{j-1} \rightarrow \operatorname{ker} \beta_{j-1}^{\prime \prime}$, that is, $\rho_{B}$ is the inclusion $\operatorname{ker} \beta_{j-1} \subset \operatorname{ker} \beta_{j-1}^{\prime \prime}$. Let $\rho_{\theta}: \operatorname{im} \theta \rightarrow \operatorname{im} \theta^{\prime \prime}$ be obtained by restricting $\rho$. Applying the snake lemma to the commutative diagram

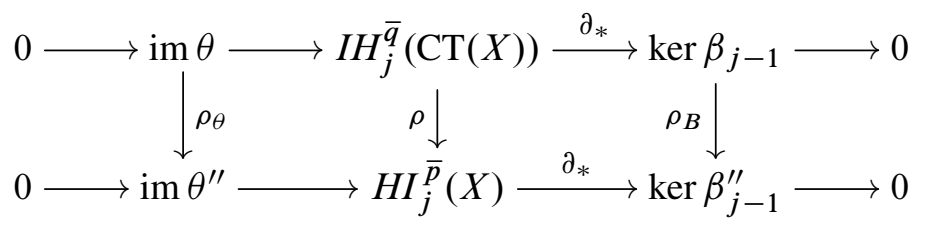

yields an exact sequence

$$
0 \rightarrow \operatorname{ker} \rho_{\theta} \rightarrow \operatorname{ker} \rho \rightarrow \operatorname{ker} \rho_{\boldsymbol{B}} \rightarrow \operatorname{coker} \rho_{\theta} \rightarrow \operatorname{coker} \rho \rightarrow \operatorname{coker} \rho_{\boldsymbol{B}} \rightarrow 0
$$

Since $\operatorname{ker} \rho_{\boldsymbol{B}}=0$, we can extract the short exact sequence

$$
0 \rightarrow \operatorname{coker} \rho_{\theta} \rightarrow \operatorname{coker} \rho \rightarrow \operatorname{coker} \rho_{B} \rightarrow 0 .
$$


As $\varphi^{\text {loc }}$ is an isomorphism, the diagram

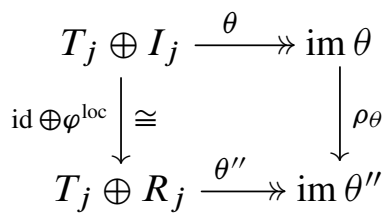

shows that $\rho_{\theta}$ is also surjective and thus coker $\rho_{\theta}=0$. Therefore, we obtain an isomorphism

$$
\operatorname{coker} \rho \stackrel{\cong}{\longrightarrow} \operatorname{coker} \rho_{B}=\frac{\operatorname{ker} \beta_{j-1}^{\prime \prime}}{\operatorname{ker} \beta_{j-1}}
$$

As $\beta_{j-1}^{\prime \prime}=\left(\beta_{j-1}^{T}, \beta_{j-1}^{R}\right)$, we have

$$
\operatorname{ker} \beta_{j-1}^{\prime \prime}=\operatorname{ker} \beta_{j-1}^{T} \cap \operatorname{ker} \beta_{j-1}^{R} \text {. }
$$

By Lemma 5.5, $\operatorname{ker} \beta_{j-1}^{J}=\operatorname{ker} \beta_{j-1}^{R}$ and hence

$$
\operatorname{coker} \rho \cong \frac{\operatorname{ker} \beta_{j-1}^{T} \cap \operatorname{ker} \beta_{j-1}^{R}}{\operatorname{ker} \beta_{j-1}^{T} \cap \operatorname{ker} \beta_{j-1}^{I}}=\frac{\operatorname{ker} \beta_{j-1}^{T} \cap \operatorname{ker} \beta_{j-1}^{J}}{\operatorname{ker} \beta_{j-1}^{T} \cap \operatorname{ker} \beta_{j-1}^{I}} \cong \operatorname{coker} \gamma
$$

Now assume that $j=1$. We need to consider the subcases $k \leq 0, k=1$ and $k>1$ separately. We start with $k \leq 0$. In principle, we shall again use a diagram of the shape of (11), but the definition of $\psi^{\text {loc }}$ changes. In this case, $L_{<k}=\varnothing$, cone $\left(f \times \mathrm{id}_{\Sigma}\right)=$ $(L \times \Sigma)^{+}, R_{0}=\mathbb{R} \oplus\left(H_{0}(L) \otimes H_{0}(\Sigma)\right), a \geq 1$ and $I_{0} \cong J_{0} \cong H_{0}(L) \otimes H_{0}(\Sigma)$. The maps of the commutative diagram

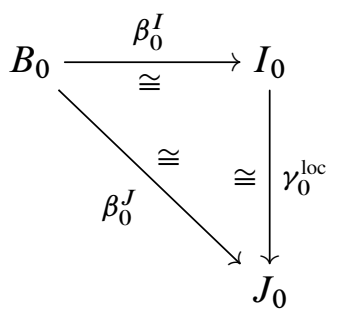

are all isomorphisms. The map $L \times \Sigma \hookrightarrow \operatorname{cone}\left(f \times \operatorname{id}_{\Sigma}\right)$, which induces $\beta^{R}$, is the inclusion $L \times \Sigma \hookrightarrow(L \times \Sigma)^{+}$and thus $\beta_{0}^{R}: B_{0} \rightarrow R_{0}$ is the standard inclusion

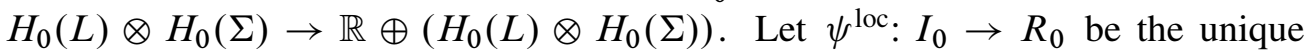


monomorphism such that

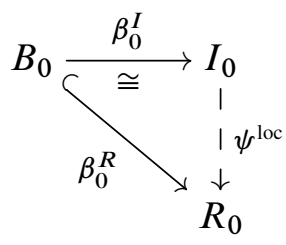

commutes. (Note that for $j \geq 2$, the map $\psi^{\text {loc }}$ was known to be surjective, which is not true here.) Diagram (11) becomes

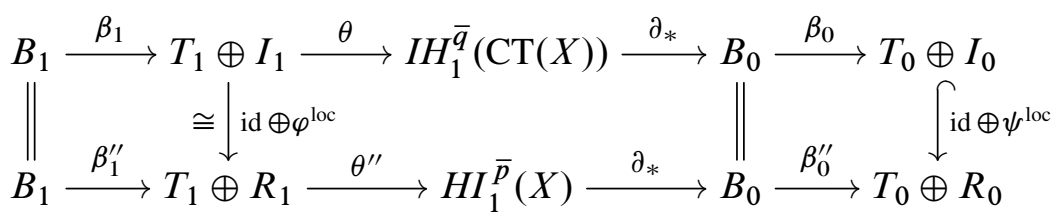

There exists a (nonunique) map $\rho: I H_{1}^{\bar{q}}(\mathrm{CT}(X)) \rightarrow H I_{1}^{\bar{p}}(X)$ which fills in the diagram commutatively. By the five lemma, $\rho$ is an isomorphism. To establish the theorem, it remains to be shown that coker $\gamma$ vanishes. Since diagram (10) is available for any $j$, the argument given in the proof of Proposition 5.6 still applies to give an isomorphism

$$
\operatorname{coker} \gamma \cong \frac{\operatorname{ker} \beta_{0}^{\prime}}{\operatorname{ker} \beta_{0}}=\frac{\operatorname{ker} \beta_{0}^{T} \cap \operatorname{ker} \beta_{0}^{J}}{\operatorname{ker} \beta_{0}^{T} \cap \operatorname{ker} \beta_{0}^{I}} .
$$

Since $\beta_{0}^{I}$ and $\beta_{0}^{J}$ are isomorphisms, we deduce that coker $\gamma=0$, as was to be shown. This concludes the case $k \leq 0$.

We proceed to the case $k=1$ (and $j=1$ ). By Lemma 5.4, $R_{0}=\mathbb{R}$, generated by the cone vertex. We have $a=0$ and thus still $I_{0}=H_{0}(L) \otimes H_{0}(\Sigma)$, but $J_{0}=0$. Therefore, $\operatorname{ker} \beta_{0}^{J}=B_{0}$. The map $\beta_{0}^{R}: B_{0} \rightarrow R_{0}$ can be identified with the augmentation map $\epsilon: B_{0} \rightarrow \mathbb{R}$, a surjection. Let $\psi^{\text {loc }}: I_{0} \rightarrow R_{0}$ be the unique epimorphism such that

$$
\begin{gathered}
B_{0} \stackrel{\beta_{0}^{I}}{\cong} I_{0} \\
1 \\
\epsilon=\beta_{0}^{R} \searrow \begin{array}{c}
\mid \text { loc } \\
\downarrow \\
R_{0}=\mathbb{R}
\end{array}
\end{gathered}
$$

commutes. There exists a map $\rho$ filling in diagram (11) commutatively. Such a $\rho$ is then injective. Using arguments from the proof of Proposition 5.6, we have

$$
\operatorname{coker} \gamma \cong \frac{\operatorname{ker} \beta_{0}^{T} \cap \operatorname{ker} \beta_{0}^{J}}{\operatorname{ker} \beta_{0}^{T} \cap \operatorname{ker} \beta_{0}^{I}}=\operatorname{ker} \beta_{0}^{T} \cap B_{0}=\operatorname{ker} \beta_{0}^{T}
$$


and

$$
\operatorname{coker} \rho \cong \operatorname{ker} \beta_{0}^{T} \cap \operatorname{ker} \beta_{0}^{R}=\operatorname{ker} \beta_{0}^{T} \cap \operatorname{ker} \epsilon .
$$

We recall from elementary algebraic topology:

Lemma 5.7 If $A$ and $B$ are topological spaces and $h: A \rightarrow B$ is a continuous map, then the diagram

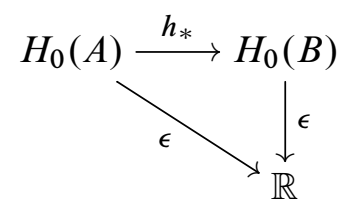

commutes. In particular, $\operatorname{ker} h_{*} \subset \operatorname{ker}\left(\epsilon: H_{0}(A) \rightarrow \mathbb{R}\right)$.

Applying this lemma to $h_{*}=\beta_{0}^{T}$, we have $\operatorname{ker} \beta_{0}^{T} \subset \operatorname{ker} \epsilon$ and thus coker $\rho \cong \operatorname{ker} \beta_{0}^{T} \cong$ coker $\gamma$. This concludes the proof in the case $k=1$.

When $k>1$ (and $j=1$ ), then $R_{0}=\mathbb{R}$ (Lemma 5.4), $a<0$, and $I_{0}=J_{0}=0$. As in the case $k=1$, the map $\beta_{0}^{R}: B_{0} \rightarrow R_{0}$ can be identified with the augmentation epimorphism $\epsilon: B_{0} \rightarrow \mathbb{R}$, but this time, there does not exist a map $\psi^{\text {loc }}$ such that $\psi^{\text {loc }} \beta_{0}^{I}=\beta_{0}^{R}$. We must therefore argue differently. By exactness and since $\beta_{0}^{I}=0$,

$$
\operatorname{im}\left(\partial_{*}: I H_{1}^{\bar{q}}(\mathrm{CT}(X)) \rightarrow B_{0}\right)=\operatorname{ker}\left(\beta_{0}^{T}, \beta_{0}^{I}\right)=\operatorname{ker} \beta_{0}^{T} .
$$

Also,

$$
\operatorname{im}\left(\partial_{*}: H I_{1}^{\bar{p}}(X) \rightarrow B_{0}\right)=\operatorname{ker}\left(\beta_{0}^{T}, \beta_{0}^{R}\right)=\operatorname{ker} \beta_{0}^{T} \cap \operatorname{ker} \epsilon .
$$

By Lemma 5.7, $\operatorname{ker} \beta_{0}^{T} \subset \operatorname{ker} \epsilon$ and hence

$$
\operatorname{im}\left(\partial_{*}: I H_{1}^{\bar{q}}(\mathrm{CT}(X)) \rightarrow B_{0}\right)=\operatorname{im}\left(\partial_{*}: H I_{1}^{\bar{p}}(X) \rightarrow B_{0}\right) .
$$

For the diagram

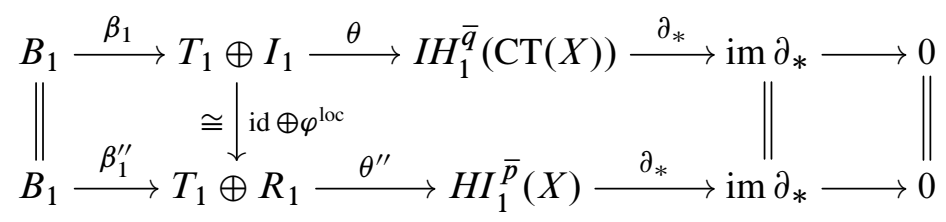

there exists a (nonunique) map $\rho: I H_{1}^{\bar{q}}(\mathrm{CT}(X)) \rightarrow H I_{1}^{\bar{p}}(X)$ which fills in the diagram commutatively. By the five lemma, $\rho$ is an isomorphism. The cokernel of $\gamma$,

$$
\operatorname{coker} \gamma \cong \frac{\operatorname{ker} \beta_{0}^{T} \cap \operatorname{ker} \beta_{0}^{J}}{\operatorname{ker} \beta_{0}^{T} \cap \operatorname{ker} \beta_{0}^{I}}=\frac{\operatorname{ker} \beta_{0}^{T} \cap B_{0}}{\operatorname{ker} \beta_{0}^{T} \cap B_{0}}=0
$$


vanishes and thus the theorem holds in this case as well. This finishes the proof for $j=1$.

It remains to establish Theorem 1.2 for $j=0$. We shall write $\widetilde{B}_{*}, \widetilde{T}_{*}$ and $\widetilde{R}_{*}$ for the reduced homology groups. We have $a=-k$,

$$
I_{0} \cong\left\{\begin{array}{cc}
H_{0}(L) \otimes H_{0}(\Sigma) & \text { if } k \leq 0 \\
0 & \text { if } k>0
\end{array}\right.
$$

and by Lemma 5.4,

$$
\widetilde{R}_{0} \cong\left\{\begin{array}{cc}
H_{0}(L) \otimes H_{0}(\Sigma) & \text { if } k \leq 0 \\
0 & \text { if } k>0
\end{array}\right.
$$

Thus $I_{0}$ and $\widetilde{R}_{0}$ are abstractly isomorphic. Recall that for a topological space $A$, the reduced homology $\tilde{H}_{0}(A)$ is the kernel of the augmentation map $\epsilon: H_{0}(A) \rightarrow \mathbb{R}$, so that there is a short exact sequence

$$
0 \rightarrow \widetilde{H}_{0}(A) \stackrel{\iota}{\rightarrow} H_{0}(A) \stackrel{\epsilon}{\rightarrow} \mathbb{R} \rightarrow 0 .
$$

Let $\widetilde{\beta}_{0}^{R}: \widetilde{B}_{0} \rightarrow \widetilde{R}_{0}$ be the map induced by $\beta_{0}^{R}$ between the kernels of the respective augmentation maps. If $k \leq 0$, then $L_{<k}=\varnothing$ and the exact sequence

$$
H_{0}\left(L_{<k} \times \Sigma\right) \rightarrow H_{0}(L \times \Sigma) \rightarrow \widetilde{R}_{0} \rightarrow 0
$$

shows that $B_{0} \rightarrow \widetilde{R}_{0}$ is an isomorphism. Since the composition

$$
\widetilde{B}_{0} \stackrel{\iota}{\hookrightarrow} B_{0} \cong \widetilde{R}_{0}
$$

is $\widetilde{\beta}_{0}^{R}$, we deduce that $\widetilde{\beta}_{0}^{R}$ is injective for $k \leq 0$. Inverting $\widetilde{\beta}_{0}^{R}$ on its image and composing with $\beta_{0}^{I}$, then extending to an isomorphism using $\operatorname{dim} I_{0}=\operatorname{dim} \widetilde{R}_{0}$, we obtain a (nonunique) isomorphism $\kappa: \widetilde{R}_{0} \rightarrow I_{0}$ such that

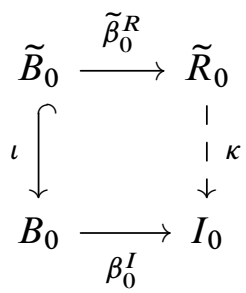

commutes. When $k>0$, let $\kappa: \widetilde{R}_{0} \rightarrow I_{0}$ be the zero map (an isomorphism). Then diagram (12) commutes also in this case. As for the above open cover $I^{\bar{p}} X=M \cup U$, the intersection $M \cap U=L \times \Sigma \times(0,1)$ is not empty, so there is a Mayer-Vietoris sequence on reduced homology:

$$
\widetilde{B}_{0} \stackrel{\beta_{0}^{\prime \prime}}{\longrightarrow} \widetilde{T}_{0} \oplus \widetilde{R}_{0} \stackrel{\theta^{\prime \prime}}{\longrightarrow} \tilde{H} I_{0}^{\bar{p}}(X) \rightarrow 0 .
$$


Using $\kappa$, we get the following commutative diagram with exact rows:

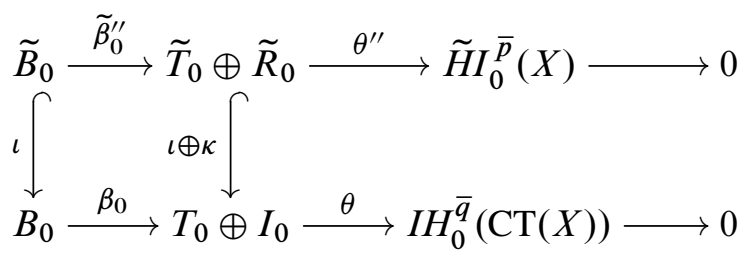

From this we infer that

$$
\widetilde{H} I_{0}^{\bar{p}}(X) \cong \operatorname{coker} \widetilde{\beta}_{0}^{\prime \prime} \quad \text { and } \quad I H_{0}^{\bar{q}}(\mathrm{CT}(X)) \cong \operatorname{coker} \beta_{0} .
$$

Let $T_{0} \oplus I_{0} \rightarrow \mathbb{R}$ be the composition of the standard projection $T_{0} \oplus I_{0} \rightarrow T_{0}$ with the augmentation $\epsilon: T_{0} \rightarrow \mathbb{R}$. Applying the snake lemma to the commutative diagram

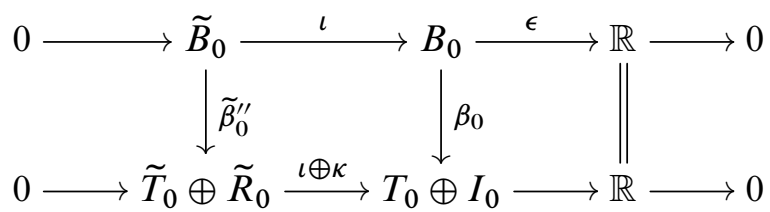

we arrive at the exact sequence

$$
0 \rightarrow \operatorname{ker} \widetilde{\beta}_{0}^{\prime \prime} \rightarrow \operatorname{ker} \beta_{0} \rightarrow \operatorname{kerid}_{\mathbb{R}} \rightarrow \operatorname{coker} \widetilde{\beta}_{0}^{\prime \prime} \rightarrow \operatorname{coker} \beta_{0} \rightarrow \operatorname{coker~id}_{\mathbb{R}} \rightarrow 0 .
$$

As ker id $\mathbb{R}_{\mathbb{R}}=0$ and coker id $\mathbb{R}_{\mathbb{R}}=0$, we obtain an isomorphism coker $\widetilde{\beta}_{0}^{\prime \prime} \cong \operatorname{coker} \beta_{0}$, ie $\widetilde{H} I_{0}^{\bar{p}}(X) \cong I H_{0}^{\bar{q}}(\mathrm{CT}(X))$. It remains to show that $\gamma: I H_{0}^{\bar{q}}(\mathrm{CT}(X)) \rightarrow I H_{0}^{\bar{q}^{\prime}}(\mathrm{CT}(X))$ is surjective. This follows from the surjectivity of $\gamma_{0}^{\text {loc }}$ and diagram (10).

\subsection{Example}

We may consider the following example to illustrate Theorem 1.2. Consider the twosphere, as a stratified space, thought of as the suspension of $S^{1}$. So the two poles are the "singular" stratum, with link $L=S^{1}$, and we will denote these by \pm pt. Now take $X=S^{2} \times T^{2}$ with the induced stratification, $\Sigma=\{ \pm \mathrm{pt}\} \times T^{2} \subset X$. The codimension of $\Sigma$ in $X$ is 2 , and any standard perversity takes $\bar{p}(2)=0$. We will first calculate $H_{*}\left(I^{\bar{p}} X\right)$. Let $\bar{M}=X-N(\Sigma)$, where $N$ is an open normal neighborhood of $\Sigma$. Note that $\partial \bar{M} \cong S^{1} \times\{ \pm \mathrm{pt}\} \times T^{2}$. The cutoff degree here is $k=1-\bar{p}(2)=1$, so $I^{\bar{p}} X=\bar{M} \cup_{g} c\left(L_{<1} \times T^{2}\right)$, where $L_{<1} \subset L=S^{1}$. For any path connected space, $L_{<1}$ is just a point $e^{0}$ in the space. Thus $g$ is the inclusion map $g: e^{0} \times\{ \pm \mathrm{pt}\} \times T^{2} \hookrightarrow \bar{M}$. The reduced homology is given by

$$
\widetilde{H} I_{*}^{\bar{p}}(X)=\widetilde{H}_{*}\left(I^{\bar{p}} X\right)=\tilde{H}_{*}\left(\bar{M}, e^{0} \times\{ \pm \mathrm{pt}\} \times T^{2}\right) .
$$


Then using the relative exact sequence on homology, we can calculate this as

$$
\tilde{H} I_{i}^{\bar{p}}(X) \cong \begin{cases}0 & \text { if } i=0 \text { or } 4, \\ \mathbb{R}^{2} & \text { if } i=1 \text { or } 3, \\ \mathbb{R}^{4} & \text { if } i=2,\end{cases}
$$

Theorem 1.2 states that there is a relationship between the $\tilde{H} I_{i}^{\bar{p}}(X)$ we just calculated and intersection homology groups for $\mathrm{CT}(X)$. The conifold transition of $X$ is $\mathrm{CT}(X)=$ $\left(\left(T^{2} \times I\right) /\left(T^{2} \times \partial I\right)\right) \times S^{1}$, whose normalization is the suspension of $T^{2}$ times $S^{1}$, that is, $\widetilde{\mathrm{CT}}(X)=S\left(T^{2}\right) \times S^{1}$. This has singular stratum $B=\{ \pm \mathrm{pt}\} \times S^{1}$ with link $F=T^{2}$, so the codimension of $B$ is 3 . This means there are two possible standard perversities $\bar{m}$ (lower middle) and $\bar{n}$ (upper middle), where $\bar{m}(3)=0$ and $\bar{n}(3)=1$. By [20, Theorem 4.2] and [19, Lemma C.1], the intersection homology does not change under normalization and thus $I H_{*}^{\bar{q}}(\mathrm{CT}(X)) \cong I H_{*}^{\bar{q}}(\widetilde{\mathrm{CT}}(X))$. The intersection homology groups of $\widetilde{\mathrm{CT}}(X)$ for the perversities $\bar{m}$ and $\bar{n}$ are calculated in [2, page 79], which also indicates the generators of the classes.

In order to illustrate Theorem 1.2, we also need to understand $I H_{*}^{\bar{q}}(\mathrm{CT}(X))$ when $\bar{q}$ is an extended perversity, as in [18], and we need to understand the maps between consecutive perversity intersection homology groups to calculate the groups $I G_{*}^{(k)}(\mathrm{CT}(X))$. By Proposition 4.1, IH $H_{*}^{\bar{q}}(\mathrm{CT}(X))=H_{*}(\bar{M})$ for $\bar{q}(3)<0$ and $I H_{*}^{\bar{q}}(\mathrm{CT}(X))=H_{*}(\bar{M}, \partial \bar{M})$ for $\bar{q}(3) \geq 2$. If $\bar{q}(3)<\bar{q}^{\prime}(3)$, then there is a natural map

$$
I H_{*}^{\bar{q}}(\mathrm{CT}(X)) \rightarrow I H_{*}^{\bar{q}^{\prime}}(\mathrm{CT}(X)),
$$

since any cycle satisfying the more restrictive condition given by $\bar{q}$ will in particular also satisfy the less restrictive condition given by $\bar{q}^{\prime}$. This is the map that appears in the definition of $I G_{*}^{(k)}(\mathrm{CT}(X))$. Now we can create Table 1 , which will allow us to calculate these groups.

\begin{tabular}{c|ccccccc}
\hline$j \backslash \bar{q}(3)$ & -1 & $\rightarrow$ & 0 & $\rightarrow$ & 1 & $\rightarrow$ & 2 \\
\hline 0 & $\mathbb{R}$ & $\cong$ & $\mathbb{R}$ & $\cong$ & $\mathbb{R}$ & $\rightarrow$ & 0 \\
1 & $\mathbb{R}^{3} \cong \mathbb{R}^{3} \rightarrow$ & $\mathbb{R}$ & 0 & $\mathbb{R}$ \\
2 & $\mathbb{R}^{3} \rightarrow$ & $\mathbb{R}^{2}$ & $\stackrel{0}{\rightarrow}$ & $\mathbb{R}^{2} \hookrightarrow$ & $\mathbb{R}^{3}$ \\
3 & $\mathbb{R}$ & $\stackrel{0}{\rightarrow}$ & $\mathbb{R}$ & $\hookrightarrow$ & $\mathbb{R}^{3} \cong$ & $\mathbb{R}^{3}$ \\
4 & 0 & $\stackrel{0}{\rightarrow}$ & $\mathbb{R}$ & $\cong$ & $\mathbb{R}$ & $\cong$ & $\mathbb{R}$ \\
\hline
\end{tabular}

Table 1: $I H_{j}^{\bar{q}}(\mathrm{CT}(X))$ 
We get, for example,

$$
\begin{aligned}
I G_{1}^{(2)}(\mathrm{CT}(X)) & \cong I H_{1}^{\bar{q}(3)=1}(\mathrm{CT}(X)) \oplus \frac{I H_{1}^{\bar{q}^{\prime}(3)=2}(\mathrm{CT}(X))}{\operatorname{im}\left(I H_{1}^{\bar{q}(3)=1}(\mathrm{CT}(X)) \rightarrow H_{1}^{\bar{q}^{\prime}(3)=2}(\mathrm{CT}(X))\right)} \\
& \cong \mathbb{R} \oplus \frac{\mathbb{R}}{0} \\
& \cong \mathbb{R}^{2} .
\end{aligned}
$$

Collecting the relevant results, and recalling that $\bar{p}(2)=0$, we get

$$
\begin{aligned}
I G_{0}^{(3)}(\mathrm{CT}(X)) & \cong 0, \\
I G_{1}^{(2)}(\mathrm{CT}(X)) & \cong \mathbb{R}^{2}, \\
I G_{2}^{(1)}(\mathrm{CT}(X)) & \cong \mathbb{R}^{4}, \\
I G_{3}^{(0)}(\mathrm{CT}(X)) & \cong \mathbb{R}^{2}, \\
I G_{4}^{(-1)}(\mathrm{CT}(X)) & \cong 0 .
\end{aligned}
$$

Thus

$$
\tilde{H} I_{j}^{\bar{p}}(X) \cong I G_{j}^{(3-j)}(\mathrm{CT}(X)),
$$

where we see $3=n-1+\bar{p}(2)$, as in Theorem 1.2.

\section{De Rham cohomology for $I H$ and $H I$}

\subsection{Extended perversities and the de Rham complex for IH}

Let $W$ be a pseudomanifold with one connected smooth singular stratum $B \subset W$ of codimension $c$ and with link $F$ of dimension $f=c-1$. (In what follows, we will take $W=\mathrm{CT}(X)$, so $B=L$ and $F=\Sigma$.) Then the only part of the perversity which affects $I H_{\bar{p}}^{*}(W)$ is the value $\bar{p}(c)$. Thus, in this special case, we can simplify notation by labelling the intersection cohomology groups by a number $p$ that depends only on the value $\bar{p}(c)$, rather than by the whole function $\bar{p}$. Further, we will fix notation such that the Poincaré lemma for a cone has the form

$$
I H_{(q)}^{j}\left(c^{\circ} F\right)=\left\{\begin{array}{cl}
H^{j}(F) & \text { if } j<q \\
0 & \text { if } j \geq q .
\end{array}\right.
$$

That is, the $q$ we use in the notation $I H_{(q)}^{j}(W)$ gives the cutoff degree in the local cohomology calculation on the link. The de Rham theorem for intersection cohomology [11] 
states that in this situation,

$$
I H_{(c-1-\bar{p}(c))}^{*}(W) \cong \operatorname{Hom}\left(I H_{*}^{\bar{p}}(W), \mathbb{R}\right) .
$$

Standard perversities satisfy $0 \leq \bar{p}(c) \leq c-2$, so in terms of the convention we have introduced, this gives $0<q \leq c-1$. We use an extension of these definitions in which $q \in \mathbb{R}$. This does not give anything dramatically new; when $q \leq 0$, we get $H^{*}(\bar{M}, \partial \bar{M})$, where $\bar{M} \cong W-N$ for $N$ an open tubular neighborhood of $B$. When $q>c-1$ we get $H^{*}(\bar{M})$. It is also worth recording that when $W$ has an isolated conical singularity $B=$ pt with link $F$, we get globally the isomorphisms

$$
I H_{(q)}^{j}(W)=\left\{\begin{array}{cl}
H^{j}(\bar{M}) & \text { if } j<q, \\
\operatorname{im}\left(H^{j}(\bar{M}, \partial \bar{M}) \rightarrow H^{j}(\bar{M})\right) & \text { if } j=q, \\
H^{j}(\bar{M}, \partial \bar{M}) & \text { if } j>q,
\end{array}\right.
$$

where in this case $\partial \bar{M} \cong F$.

In order to prove Theorem 1.3 from the de Rham perspective, we need to use compatible de Rham complexes to define these cohomologies. Various complexes have been shown to calculate intersection cohomology of a pseudomanifold. We will present first a version of the de Rham complex from [11], adapted to our setting.

We use the notation from Section 2, and in particular let $W=\mathrm{CT}(X)$ have an $l-$ dimensional smooth singular stratum $L$ with link $\Sigma$, a smooth $s$-dimensional manifold, and product link bundle $Y \cong L \times \Sigma$. Note that $s+1=\operatorname{codim} L$. From the isomorphism $Y \cong L \times \Sigma$, we have that $T^{*} Y \cong T^{*} L \oplus T^{*} \Sigma$. This induces a bundle splitting

$$
\Lambda^{k}\left(T^{*} Y\right) \cong \bigoplus_{i+j=k} \Lambda^{i}\left(T^{*} L\right) \otimes \Lambda^{j}\left(T^{*} \Sigma\right)
$$

We write $\Omega^{k}(Y)=\Gamma^{\infty}\left(Y ; \Lambda^{k}\left(T^{*} Y\right)\right)$ for the space of smooth differential $k$-forms on $Y$, and we write $\Lambda^{i, j} Y=\Lambda^{i}\left(T^{*} L\right) \otimes \Lambda^{j}\left(T^{*} \Sigma\right)$ and $\Omega^{i, j}(Y)=\Gamma^{\infty}\left(Y ; \Lambda^{i, j} Y\right)$. Then also we obtain a splitting of the space of smooth sections,

$$
\Omega^{k}(Y) \cong \bigoplus_{i+j=k} \Omega^{i, j}(Y), \quad \alpha=\sum_{i, j} \alpha_{i, j}
$$

as $C^{\infty}(Y)$-modules. For $q \in \mathbb{Z}$, define the fibrewise (along $\Sigma$ ) truncated space of forms over $Y$ by

$$
\left(\mathrm{ft}_{<q} \Omega^{*}(Y)\right)^{k}:=\left\{\alpha \in \Omega^{k}(Y) \mid \alpha=\sum_{j=0}^{q-1} \alpha_{k-j, j}\right\} .
$$


Note that $\mathrm{ft}_{<q} \Omega^{*}(Y)$ is not a complex in general. Then define the complex

$$
\begin{aligned}
& I \Omega_{(q)}^{*}(\mathrm{CT}(X)) \\
& \quad:=\left\{\omega \in \Omega^{*}(\bar{M}) \mid \operatorname{inc}^{*} \omega \in \mathrm{ft}_{<q} \Omega^{*}(Y) \text { and inc }{ }^{*}(d \omega) \in \mathrm{ft}_{<q} \Omega^{*}(Y)\right\} .
\end{aligned}
$$

(Recall that inc: $Y=\partial \bar{M} \hookrightarrow \bar{M}$ is the inclusion of the boundary.) The cohomology of this complex is $I H_{(q)}^{*}(\mathrm{CT}(X))$, as shown in eg [11]. We note that there is an inclusion of complexes,

$$
S_{q, q+1}: I \Omega_{(q)}^{*}(\mathrm{CT}(X)) \hookrightarrow I \Omega_{(q+1)}^{*}(\mathrm{CT}(X)) .
$$

This induces a natural map on cohomology, which however is generally neither injective nor surjective. We will come back to this map later.

\subsection{Extended perversities and the de Rham complex for $H I$}

In this subsection, we present the de Rham complex defined in [4], which computes the reduced singular cohomology of intersection spaces. Let $L$ be oriented and equipped with a Riemannian metric. For flat link bundles $E \rightarrow \Sigma$ whose link can be given a Riemannian metric such that the transition functions are isometries, the first author defined in [4] a subcomplex $\Omega_{\mathcal{M S}}^{*}(\Sigma) \subset \Omega^{*}(E)$, the complex of multiplicatively structured forms. In the present special case of $E=Y=L \times \Sigma$, this subcomplex is

$$
\Omega_{\mathcal{M S}}^{*}(\Sigma)=\left\{\omega \in \Omega^{*}(Y) \mid \omega=\sum_{i, j} \pi_{L}^{*} \lambda_{i} \wedge \pi_{\Sigma}^{*} \sigma_{j}\right\},
$$

where the sum here is finite, and $\lambda_{i} \in \Omega^{*}(L)$ and $\sigma_{j} \in \Omega^{*}(\Sigma)$. We may also write this as

$$
\Omega_{\mathcal{M S}}^{*}(\Sigma) \cong \Omega^{*}(L) \otimes \Omega^{*}(\Sigma)
$$

Let $k$ be any integer. The level- $k$ cotruncation of the complex $\Omega^{*}(L)$ is defined in loc. cit. as the subcomplex $\tau_{\geq k} \Omega^{*}(L) \subset \Omega^{*}(L)$ given in degree $m$ by

$$
\left(\tau_{\geq k} \Omega^{*}(L)\right)^{m}=\left\{\begin{array}{cc}
0 & \text { if } m<k, \\
\operatorname{ker} \delta_{L} & \text { if } m=k, \\
\Omega^{m}(L) & \text { if } m>k,
\end{array}\right.
$$

using the codifferential $\delta_{L}$ on $\Omega^{*}(L)$. Note that $\tau_{\geq k} \Omega^{*}(L)=\Omega^{*}(L)$ for $k \leq 0$, while $\tau_{\geq k} \Omega^{*}(L)=0$ for $k>l$. The subcomplex $\mathrm{ft}_{\geq k} \Omega_{\mathcal{M S}}^{*}(\Sigma) \subset \Omega_{\mathcal{M S}}^{*}(\Sigma)$ of fibrewise 
(along $L$ ) cotruncated forms is given in degree $m$ by

$$
\left(\mathrm{ft}_{\geq k} \Omega_{\mathcal{M S}}^{*}(\Sigma)\right)^{m}=\left\{\omega \in \Omega^{m}(Y) \mid \omega=\sum_{i, j} \pi_{L}^{*} \lambda_{i} \wedge \pi_{\Sigma}^{*} \sigma_{j}, \lambda_{i} \in \tau_{\geq k} \Omega^{*}(L)\right\} .
$$

Taking $k=l-\bar{p}(l+1)$, we set

$$
H I_{\mathrm{dR}, \bar{p}}^{*}(X):=H^{*}\left(\Omega I_{\bar{p}}^{*}(M)\right),
$$

where

$$
\Omega I_{\bar{p}}^{*}(M):=\left\{\omega \in \Omega^{*}(M)|\omega|_{N-\Sigma}=\pi_{Y}^{*} \eta, \eta \in \mathrm{ft}_{\geq l-\bar{p}(l+1)} \Omega_{\mathcal{M S}}^{*}(\Sigma)\right\} .
$$

(Recall from Section 2 that $N$ is an open tubular neighborhood of $\Sigma$ with a fixed diffeomorphism $N-\Sigma \cong L \times \Sigma \times(0,1)$ and $\pi_{Y}: N-\Sigma \rightarrow Y=L \times \Sigma$ is the projection.) The Poincaré duality theorem of [4] asserts that if $\bar{p}$ and $\bar{q}$ are complementary perversities, then wedge product of forms followed by integration induces a nondegenerate bilinear form

$$
H I_{\mathrm{dR}, \bar{p}}^{*}(X) \times H I_{\mathrm{dR}, \bar{q}}^{n-*}(X) \rightarrow \mathbb{R}, \quad([\omega],[\eta]) \mapsto \int_{X-\Sigma} \omega \wedge \eta,
$$

when $X$ is compact and oriented. (This is shown not just for trivial link bundles, but for any flat link bundle whose transition functions are isometries of the link.) Furthermore, using a certain partial smoothing technique that we shall sketch below, the de Rham theorem of [4] for isolated singularities and its generalization to nonisolated singularities with trivial link bundle due to Essig [16, Theorem 3.4.1] asserts that

$$
H I_{\mathrm{dR}, \bar{p}}^{*}(X) \cong \operatorname{Hom}\left(\widetilde{H}_{*}\left(I^{\bar{p}} X ; \mathbb{R}\right), \mathbb{R}\right) .
$$

This isomorphism is constructed as follows: For a topological space $Z$, let $C_{*}(Z)$ denote its singular chain complex with real coefficients. For a smooth manifold $V$ (which is allowed to have a boundary), let $C_{*}^{\infty}(V)$ denote its smooth singular chain complex with real coefficients, generated by smooth singular simplices $\Delta^{j} \rightarrow V$. For a continuous map $g: Z \rightarrow V$, the first author defined in [4] the partially smooth chain complex $C_{*}^{\propto}(g)$. In degree $j$,

$$
C_{j}^{\alpha}(g)=H_{j-1}(Z) \oplus C_{j}^{\infty}(V) .
$$

Let $\iota: C_{*}^{\infty}(V) \hookrightarrow C_{*}(V)$ be the inclusion and $s: C_{*}(V) \rightarrow C_{*}^{\infty}(V)$ be Lee's smoothing operator [29, pages 416-424]. The map $s$ is a chain map such that $s \circ \iota$ is the identity and $\iota \circ s$ is chain homotopic to the identity. Thus $s$ and $\iota$ induce mutually inverse 
isomorphisms on homology. If $V$ has a nonempty boundary $\partial V$, then we can assume that $s$ has been arranged so that the square

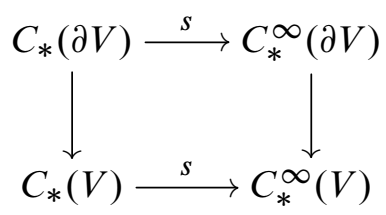

commutes. Let $Z_{j}$ denote the subspace of $j$-cycles in $C_{j}(Z)$ and $B_{j}=\partial C_{j+1}(Z)$ the subspace of $j$-boundaries. Choosing direct sum decompositions $Z_{j}=B_{j} \oplus H_{j}^{\prime}$, we obtain a quasi-isomorphism $q: H_{*}(Z)=H_{*}\left(C_{*}(Z)\right) \rightarrow C_{*}(Z)$, which is given in degree $j$ by the composition

$$
H_{j}(Z)=\frac{Z_{j}}{B_{j}}=\frac{B_{j} \oplus H_{j}^{\prime}}{B_{j}} \cong H_{j}^{\prime} \hookrightarrow Z_{j} \hookrightarrow C_{j}(Z) .
$$

Here, we regard $H_{*}(Z)$ as a chain complex with zero boundary operators. By construction, the formula $[q(z)]=z$ holds for a homology class $z \in H_{j}(Z)$. Let $z \in H_{j-1}(Z)$ be a homology class in $Z$ and $v: \Delta^{j} \rightarrow V$ be a smooth singular simplex $v \in C_{j}^{\infty}(V)$. The boundary operator $\partial: C_{j}^{\propto}(g) \rightarrow C_{j-1}^{\propto}(g)$ is defined to be

$$
\partial(z, v)=\left(0, \partial v+s g_{*} q(z)\right)
$$

where $g_{*}: C_{j-1}(Z) \rightarrow C_{j-1}(V)$ is the chain map induced by $g$. By Proposition 9.2 of [4], the partially smooth chain complex $C_{*}^{\propto}(g)$ is naturally quasi-isomorphic to the algebraic mapping cone $C_{*}\left(g_{*}\right)$ of $g_{*}$. Applying this to the map $g: L_{<k} \times \Sigma \rightarrow \bar{M}$, we get an identification

$$
H_{*}\left(C_{*}^{\propto}(g)\right) \cong H_{*}\left(C_{*}\left(g_{*}\right)\right)=\tilde{H}_{*}(\operatorname{cone}(g))=\tilde{H}_{*}\left(I^{\bar{p}} X\right) .
$$

A map

$$
\Psi_{\bar{p}}: H^{j}\left(\Omega I_{\bar{p}}^{*}(M)\right) \rightarrow \operatorname{Hom}\left(H_{j}\left(C_{*}^{\propto}(g)\right), \mathbb{R}\right)
$$

is given by $\Psi_{\bar{p}}[\omega][(z, v)]=\int_{v} \omega$, where $\omega \in \Omega I_{\bar{p}}^{j}(M)$ is a closed form and $(z, v) \in$ $C_{j}^{\propto}(g)$ is a cycle. (See [4, page 48]. Note that $\omega$ has a unique extension to a closed form on $\bar{M}$; see also Section 6.3.) Then the isomorphism (15) is the composition

$$
H I_{\mathrm{dR}, \bar{p}}^{*}(X) \stackrel{\Psi_{\bar{p}}}{\rightarrow} \operatorname{Hom}\left(H_{*}\left(C_{*}^{\propto}(g)\right), \mathbb{R}\right) \cong \operatorname{Hom}\left(\tilde{H}_{*}\left(I^{\bar{p}} X\right), \mathbb{R}\right)
$$

This construction, as well as the argument showing the map to be an isomorphism, does not require any assumptions on the cutoff degree $k \in \mathbb{Z}$, and thus works even for extended perversities $\bar{p}$. We can create a notation for $H I_{\mathrm{dR}, \bar{p}}^{*}$ that emphasises 
the cutoff degree instead of the perversity in a similar vein to the notation we fixed for $I H_{\bar{p}}^{*}(X)$ in the previous section. With $k=l-\bar{p}(l+1)$, we simply write

$$
H I_{(k)}^{*}(X):=H I_{\mathrm{dR}, \bar{p}}^{*}(X) .
$$

Since the only value of $\bar{p}$ to make a difference in the right side of this equation is $\bar{p}(l+1)$, no ambiguity arises from replacing the function $\bar{p}$ by the number $k$, where now $k$ is giving the cutoff degree in the local calculation on the link.

We observe two useful lemmas about the cohomology of the complex $\Omega I_{\bar{p}}^{*}(M)$. The first one is a generalized Mayer-Vietoris sequence.

Lemma 6.1 There is a long exact sequence of de Rham cohomology groups as follows:

$$
\cdots \rightarrow H I_{(k)}^{j}(X) \rightarrow H^{j}(\bar{M}) \oplus H^{j}\left(\mathrm{ft}_{\geq k} \Omega_{\mathcal{M S}}^{*}(\Sigma)\right) \rightarrow H^{j}(\partial \bar{M}) \rightarrow \cdots .
$$

In particular, since $\partial \bar{M}=L \times \Sigma$, the second summand of the middle term is isomorphic to

$$
\bigoplus_{i=k}^{l} H^{i}(L) \otimes H^{j-i}(\Sigma) .
$$

Proof Let $k=l-\bar{p}(l+1)$. By definition of $\Omega I_{\bar{p}}^{*}(M)$, we have a short exact sequence of complexes

$$
0 \rightarrow \Omega I_{\bar{p}}^{*}(M) \rightarrow \Omega^{*}(M) \oplus \mathrm{ft}_{\geq k} \Omega_{\mathcal{M S}}^{*}(\Sigma) \rightarrow \Omega^{*}(N-\Sigma) \rightarrow 0,
$$

where the second map takes a pair $(\omega, \eta)$ to $\left.\omega\right|_{N-\Sigma}-\pi_{Y}^{*} \eta$, and the first map takes $\omega \in \Omega I_{\bar{p}}^{*}(M)$ with $\left.\omega\right|_{N-\Sigma}=\pi_{Y}^{*} \eta$ to $(\omega, \eta)$. This sequence induces a long exact sequence on cohomology isomorphic to the one above. The form of the second summand comes from the definition of cotruncation and of multiplicatively structured forms. The isomorphism of the third term comes from the standard de Rham Künneth isomorphism $H^{j}(\partial \bar{M}) \cong H^{j}(\partial \bar{M} \times(0,1))$ and the diffeomorphism $N-\Sigma \cong \partial \bar{M} \times(0,1)$.

Lemma 6.2 (Künneth for $H I_{*}$ ) If $W$ is a pseudomanifold with only one isolated singularity and $B$ is a closed manifold, then the homological cross product induces an isomorphism $\tilde{H} I_{*}^{\bar{p}}(W \times B) \cong \widetilde{H} I_{*}^{\bar{p}}(W) \otimes H_{*}(B)$.

Proof Let $\bar{W}$ be the blowup of $W$. Set $k=l-\bar{p}(l+1)$, where $l$ is the dimension of the link $L=\partial \bar{W}$, and let $f: L_{<k} \rightarrow L$ be a stage- $k$ Moore approximation to $L$. Then $I^{\bar{p}} W=$ cone $(g)$, where $g$ is the composition

$$
L_{<k} \stackrel{f}{\rightarrow} L=\partial \bar{W} \hookrightarrow \bar{W} .
$$


The blowup $\bar{M}$ of $X=W \times B$ is $\bar{M}=\bar{W} \times B$ with boundary $\partial \bar{M}=L \times B$. The intersection space of $X$ is then $I^{\bar{p}} X=\operatorname{cone}\left(g \times \mathrm{id}_{B}\right)$ because $g \times \mathrm{id}_{B}$ is the composition

$$
L_{<k} \times B \stackrel{f \times \mathrm{id}_{B}}{\longrightarrow} L \times B=\partial \bar{M} \hookrightarrow \bar{M}=\bar{W} \times B .
$$

Let $v \in \operatorname{cone}(g)$ be the cone vertex and let $Q=(\operatorname{cone}(g) \times B) /(\{v\} \times B)$, which is homeomorphic to cone $\left(g \times \operatorname{id}_{B}\right)$. As the inclusion $\{v\} \times B \rightarrow \operatorname{cone}(g) \times B$ is a closed cofibration, the quotient map induces an isomorphism

$H_{*}((\operatorname{cone}(g),\{v\}) \times B)=H_{*}(\operatorname{cone}(g) \times B,\{v\} \times B) \cong \widetilde{H}_{*}(Q) \cong \widetilde{H}_{*}\left(\operatorname{cone}\left(g \times \operatorname{id}_{B}\right)\right)$.

The Künneth theorem for relative homology [34, Theorem 5.3.10, page 235] asserts that the cross product

$$
H_{*}(\operatorname{cone}(g),\{v\}) \otimes H_{*}(B) \stackrel{\times}{\longrightarrow} H_{*}((\operatorname{cone}(g),\{v\}) \times B)
$$

is an isomorphism. Composing, we obtain an isomorphism

$$
\begin{aligned}
\tilde{H} I_{*}^{\bar{p}}(W \times B) & =\widetilde{H}_{*}\left(\operatorname{cone}\left(g \times \operatorname{id}_{B}\right)\right) \cong H_{*}((\operatorname{cone}(g),\{v\}) \times B) \\
& \cong \widetilde{H}_{*}(\operatorname{cone}(g)) \otimes H_{*}(B)=\tilde{H} I_{*}^{\bar{p}}(W) \otimes H_{*}(B) .
\end{aligned}
$$

Lemma 6.3 (Künneth for $H I_{\mathrm{dR}}^{*}$ ) Let $W$ be a pseudomanifold with only one isolated singularity and $B$ be a smooth closed manifold such that $\tilde{H} I_{*}^{\bar{p}}(W)$ and $H_{*}(B)$ are finite-dimensional. Then $H I_{\mathrm{dR}, \bar{p}}^{*}(W \times B) \cong H I_{\mathrm{dR}, \bar{p}}^{*}(W) \otimes H^{*}(B)$.

Proof The homology groups $\tilde{H} I_{*}^{\bar{p}}(W)$ and $H_{*}(B)$ are finite-dimensional by assumption, and thus the natural map

$$
\operatorname{Hom}\left(\tilde{H} I_{*}^{\bar{p}}(W), \mathbb{R}\right) \otimes \operatorname{Hom}\left(H_{*}(B), \mathbb{R}\right) \rightarrow \operatorname{Hom}\left(\tilde{H} I_{*}^{\bar{p}}(W) \otimes H_{*}(B), \mathbb{R} \otimes \mathbb{R}\right)
$$

is an isomorphism. Thus, by Lemma 6.2 and the de Rham isomorphism (15),

$$
\begin{aligned}
H I_{\mathrm{dR}, \bar{p}}^{*}(W \times B) & \cong \operatorname{Hom}\left(\tilde{H} I_{*}^{\bar{p}}(W \times B), \mathbb{R}\right) \\
& \cong \operatorname{Hom}\left(\tilde{H} I_{*}^{\bar{p}}(W) \otimes H_{*}(B), \mathbb{R}\right) \\
& \cong \operatorname{Hom}\left(\tilde{H} I_{*}^{\bar{p}}(W), \mathbb{R}\right) \otimes \operatorname{Hom}\left(H_{*}(B), \mathbb{R}\right) \\
& \cong H I_{\mathrm{dR}, \bar{p}}^{*}(W) \otimes H^{*}(B) .
\end{aligned}
$$

Using these lemmas, we may also compute $H I_{(k)}^{*}(X)$ for values of extended perversities that lie outside of the topologically invariant range of Goresky-MacPherson, as follows: 
For standard perversities, $1 \leq k \leq l$. If $k \leq 0$, then $\tau_{\geq k} \Omega^{*}(L)=\Omega^{*}(L)$ and thus the sequence of Lemma 6.1 becomes

$$
\cdots \rightarrow H I_{(k)}^{j}(X) \stackrel{\phi}{\rightarrow} H^{j}(\bar{M}) \oplus H^{j}\left(\Omega_{\mathcal{M S}}^{*}(\Sigma)\right) \stackrel{\psi}{\rightarrow} H^{j}(\partial \bar{M}) \rightarrow \cdots .
$$

The map $\psi$ has the form $\psi=\psi_{M}-\psi_{Y}$, where $\psi_{M}: H^{j}(\bar{M}) \rightarrow H^{j}(\partial \bar{M})$ is restriction and $\psi_{Y}: H^{j}\left(\Omega_{\mathcal{M S}}^{*}(\Sigma)\right) \rightarrow H^{j}(\partial \bar{M})$ is induced by the inclusion of complexes. Since $\psi_{Y}$ is in the present case an isomorphism, the map $\psi$ is surjective and thus $\phi$ is injective. Now

$$
\operatorname{im} \phi=\operatorname{ker} \psi=\left\{(\omega, \eta) \mid \psi_{M}(\omega)=\psi_{Y}(\eta)\right\}=\left\{\left(\omega, \psi_{Y}^{-1} \psi_{M}(\omega)\right)\right\},
$$

which is isomorphic to $H^{*}(\bar{M})$. We conclude that $H I_{(k)}^{*}(X) \cong H^{*}(\bar{M})$ when $k \leq 0$. On the other hand, if $k>l$, then $\tau_{\geq k} \Omega^{*}(L)=0$ and hence the sequence of Lemma 6.1 becomes

$$
\cdots \rightarrow H I_{(k)}^{j}(X) \rightarrow H^{j}(\bar{M}) \rightarrow H^{j}(\partial \bar{M}) \rightarrow \cdots .
$$

Therefore, $H I_{(k)}^{*}(X) \cong H^{*}(\bar{M}, \partial \bar{M})$ when $k>l$. In particular, Poincaré duality also works for these extended perversities, since relative and absolute (co)homology pair nondegenerately under the standard intersection pairing.

\subsection{An alternative de Rham complex for $\mathrm{HI}$}

We continue to assume that $L$ is oriented. We now want to define a new, equivalent de Rham complex for $H I_{(k)}^{*}(X)$ that is analogous to the de Rham complex we presented above for $I H_{(q)}^{*}(\mathrm{CT}(X))$. In order to do this, we need to extend the operator $\delta_{L}$ from multiplicatively structured forms on $Y$ to all smooth forms on $Y$. This is standard, but we give details here for clarity. First, we can decompose the exterior derivative according to the splitting of $Y$ as

$$
d_{Y}=\tilde{d}_{L}+(-1)^{r} \tilde{d}_{\Sigma}
$$

for $(r, *)$-forms, where for $z=\left(z_{1}, \ldots, z_{l}\right)$ local coordinates on a coordinate patch $U \subset L$ and $\boldsymbol{y}=\left(y_{1}, \ldots, y_{s}\right)$ local coordinates on a coordinate patch $V \subset \Sigma$ and multi-indices $\boldsymbol{I}$ and $\boldsymbol{J}$,

$$
\begin{aligned}
& \tilde{d}_{L}\left(f(\boldsymbol{z}, \boldsymbol{y}) d z_{\boldsymbol{I}} \wedge d y_{\boldsymbol{J}}\right):=\sum_{i=1}^{l} \frac{\partial f}{\partial z_{i}} d z_{i} \wedge d z_{\boldsymbol{I}} \wedge d y_{\boldsymbol{J}}, \\
& \tilde{d}_{\Sigma}\left(f(\boldsymbol{z}, \boldsymbol{y}) d z_{\boldsymbol{I}} \wedge d y_{\boldsymbol{J}}\right):=\sum_{j=1}^{s} \frac{\partial f}{\partial y_{j}} d z_{\boldsymbol{I}} \wedge d y_{j} \wedge d y_{\boldsymbol{J}} .
\end{aligned}
$$


Note $\tilde{d}_{L}\left(\pi_{L}^{*} \lambda \wedge \pi_{\Sigma}^{*} \sigma\right)=\pi_{L}^{*}\left(d_{L} \lambda\right) \wedge \pi_{\Sigma}^{*} \sigma$ and $\tilde{d}_{\Sigma}\left(\pi_{L}^{*} \lambda \wedge \pi_{\Sigma}^{*} \sigma\right)=\pi_{L}^{*} \lambda \wedge \pi_{\Sigma}^{*}\left(d_{\Sigma} \sigma\right)$, so these operators extend the exterior derivatives on multiplicatively structured forms to operators over all smooth forms on $Y$.

Now fix a metric $g_{L}$ on $L$. This defines a Hodge star operator on $\Omega^{*}(L)$, which may be extended to forms on $Y$ via the rule

$$
\widetilde{*}_{L}\left(d z_{I} \wedge d y_{J}\right):=\left(*_{L} d z_{I}\right) \wedge d y_{J} .
$$

We can extend the adjoint operator of $d_{L}$ to forms on $Y$ by setting for $(i, j)$-forms that

$$
\tilde{\delta}_{L}:=(-1)^{l i+l+1} \widetilde{*}_{L} \tilde{d}_{L} \widetilde{*}_{L} .
$$

Note that this does extend the adjoint operator from multiplicatively structured forms. From the coordinate definitions and the invariance of $g_{L}$ in the $V$ coordinates, observe

$$
\tilde{d}_{L} \tilde{d}_{\Sigma}=\tilde{d}_{\Sigma} \tilde{d}_{L}, \quad \tilde{d}_{\Sigma} \tilde{*}_{L}=\widetilde{*}_{L} \tilde{d}_{\Sigma}, \quad \text { and } \quad \tilde{d}_{\Sigma} \tilde{\delta}_{L}=\tilde{\delta}_{L} \tilde{d}_{\Sigma} \text {. }
$$

Further, we can lift the Hodge decomposition for $\Omega^{*}(L)$ to any neighborhood $L \times V$ by observing that for any fixed $y \in V$, we have a decomposition of $\omega \in \Omega^{i, j}(L \times V)$ given by

$$
\omega=\sum_{|J|=j} \lambda_{J}(\boldsymbol{y}) \wedge d y_{J}
$$

where each $\lambda_{J}(\boldsymbol{y}) \in \Omega^{i}(L)$ decomposes as $d_{L} \lambda_{1, J}(y)+\delta_{L} \lambda_{2, J}(y)+\lambda_{3, J}(y)$, with $\lambda_{3, J}(y) \in \mathcal{H}^{i}(L)$. Here, $\mathcal{H}^{*}(L)$ denotes the space of harmonic forms on $L$. Thus altogether we can decompose

$$
\text { (17) } \begin{aligned}
\omega & =\sum_{|J|=j} d_{L} \lambda_{1, J}(\boldsymbol{y}) \wedge d y_{J}+\sum_{|J|=j} \delta_{L} \lambda_{2, J}(\boldsymbol{y}) \wedge d y_{J}+\sum_{|J|=j} \lambda_{3, J}(\boldsymbol{y}) \wedge d y_{J} \\
& =\tilde{d}_{L} \sum_{|J|=j} \lambda_{1, J}(\boldsymbol{y}) \wedge d y_{J}+\tilde{\delta}_{L} \sum_{|J|=j} \lambda_{2, J}(\boldsymbol{y}) \wedge d y_{J}+\sum_{|J|=j} \lambda_{3, J}(\boldsymbol{y}) \wedge d y_{J} \\
& =\tilde{d}_{L} \omega_{1}(\boldsymbol{y})+\tilde{\delta}_{L} \omega_{2}(\boldsymbol{y})+\omega_{3}(\boldsymbol{y}) .
\end{aligned}
$$

Now putting this together for all $y \in V$, we get a unique decomposition of any form in $\Omega^{i, j}(L \times V)$ into pieces in the image of $\widetilde{d}_{L}$, in the image of $\widetilde{\delta}_{L}$ and in the kernel of both.

Lemma 6.4 We have the decomposition

$$
\Omega^{i, j} Y=\tilde{d}_{L} \Omega^{i-1, j}(Y) \oplus \tilde{\delta}_{L} \Omega^{i+1, j}(Y) \oplus\left(\mathcal{H}^{i}(L) \otimes \Omega^{j}(\Sigma)\right),
$$

where the sums are vector space direct sums. Further, $\tilde{d}_{\Sigma}$ preserves this decomposition. 
Proof We have already demonstrated the decomposition, since this is done pointwise in $B$ (finite-dimensionality of $\mathcal{H}^{i}(L)$ allows us to write the last term of the decomposition as a tensor product). The fact that it is preserved by $\tilde{d}_{\Sigma}$ follows from (16).

Note that since $L \times \Sigma$ is a product, we can also apply Lemma 6.4 in the other direction, namely that $\tilde{d}_{L}$ preserves the Hodge decomposition for $\Sigma$. In this way, we get in fact a double Hodge decomposition. A graded vector space $\widehat{\mathrm{ft}}_{\geq k} \Omega^{*}(Y)$ of alternatively fibrewise cotruncated forms is given by

$$
\left(\widehat{\mathrm{ft}}_{\geq k} \Omega^{*}(Y)\right)^{j}:=\left\{\alpha=\sum_{i=k}^{l} \alpha_{i, j-i} \mid \alpha_{i, j-i} \in \Omega^{i, j-i}(Y), \tilde{\delta}_{L} \alpha_{k, j-k}=0\right\} .
$$

Lemma 6.5 The differential $d_{Y}$ restricts to $\widehat{\mathrm{ft}}_{\geq k} \Omega^{*}(Y)$.

Proof The differential $d_{Y}=\tilde{d}_{L} \pm \tilde{d}_{\Sigma}$ does not lower the $L$-degree $i$ of a form $\alpha_{i, j-i}$. Thus, if $\alpha=\sum_{i=k}^{l} \alpha_{i, j-i}$, then $d_{Y} \alpha$ can again be written in the form $d_{Y} \alpha=$ $\sum_{i=k}^{l} \beta_{i, j+1-i}$, where $\beta_{i, j+1-i} \in \Omega^{i, j+1-i}(Y)$. Assume that $\tilde{\delta}_{L} \alpha_{k, j-k}=0$. Since

$$
\begin{aligned}
d_{Y} \alpha & =\left(\tilde{d}_{L} \pm \tilde{d}_{\Sigma}\right)\left(\alpha_{k, j-k}+\alpha_{k+1, j-k-1}+\cdots\right) \\
& =\tilde{d}_{L} \alpha_{k, j-k} \pm \tilde{d}_{\Sigma} \alpha_{k, j-k}+\tilde{d}_{L} \alpha_{k+1, j-k-1} \pm \tilde{d}_{\Sigma} \alpha_{k+1, j-k-1}+\cdots,
\end{aligned}
$$

the component in bidegree $(k, j+1-k)$ of $d_{Y} \alpha$ is

$$
\left(d_{Y} \alpha\right)_{k, j+1-k}= \pm \tilde{d}_{\Sigma} \alpha_{k, j-k}
$$

Using (16),

$$
\tilde{\delta}_{L}\left(d_{Y} \alpha\right)_{k, j+1-k}= \pm \tilde{\delta}_{L} \tilde{d}_{\Sigma} \alpha_{k, j-k}= \pm \tilde{d}_{\Sigma} \tilde{\delta}_{L} \alpha_{k, j-k}=0 .
$$

This shows that $d_{Y} \alpha \in\left(\widehat{\mathrm{ft}}_{\geq k} \Omega^{*}(Y)\right)^{j+1}$.

By the lemma, $\widehat{\mathrm{ft}}_{\geq k} \Omega^{*}(Y)$ is a differential complex. Now we can define the new de Rham complex for $H I_{(k)}^{*}(X)$ as

$$
\widehat{\Omega I}_{(k)}^{*}(X):=\left\{\left.\mu\right|_{M} \mid \mu \in \Omega^{*}(\bar{M}), \text { inc }^{*}(\mu) \in \widehat{\mathrm{ft}}_{\geq k} \Omega^{*}(Y)\right\} .
$$

We want to show this complex is quasi-isomorphic to the original de Rham complex. For this, we will need the Künneth theorem.

Theorem 6.6 (Künneth theorem) Let $Y=L \times \Sigma$, where $\Sigma$ has a finite good cover. Then the inclusion of complexes

$$
\Omega^{*}(L) \otimes \Omega^{*}(\Sigma) \cong \Omega_{\mathcal{M S}}(\Sigma) \hookrightarrow \Omega^{*}(Y)
$$


induces an isomorphism on cohomology. In particular, if $\alpha \in \Omega_{\mathcal{M S}}^{*}(\Sigma)$ and $\alpha=d \beta$ for $\beta \in \Omega^{*}(Y)$, then $\beta=d \gamma+\beta^{\prime}$, where $\beta^{\prime} \in \Omega_{\mathcal{M S}}^{*}(\Sigma)$.

Note that the second part of the Künneth theorem is not obvious, but follows from the standard proof for the de Rham cohomology version. Details may be found eg in [10, page 49].

It is also useful to start with two observations. First, although the forms in the complex $\Omega I_{(k)}^{*}(X)$ are only defined on $M$, we can extend each one uniquely to a form on $\bar{M}$. This is because on $N-\Sigma \cong \partial \bar{M} \times(0,1)$, they are constant in the variable on $(0,1)$ (which we will call $x$ ), and thus can be extended to $\bar{M} \times[0,1)$. We will abuse notation and use $\Omega I_{(k)}^{*}(X)$ to denote both the original complex and the complex of extended forms, which is isomorphic to the original complex through the extension map. This is convenient, because it then allows us to consider $\Omega I_{(k)}^{*}(X)$ as a subcomplex of $\widehat{\Omega I}_{(k)}^{*}(X)$.

Second, we observe that when we choose our neighborhood $N$ of $\Sigma \subset X$, we can always arrange for it to sit inside a larger neighborhood $N_{2} \subset X$ with the property that $N_{2}-\Sigma \cong \partial \bar{M} \times(0,2)$, where $N-\Sigma \cong \partial \bar{M} \times(0,1)$ is a restriction of the diffeomorphism for $N_{2}-\Sigma$. Let $\bar{N}$ denote the blowup of $N$, so that $\bar{N} \cong \partial \bar{M} \times[0,1)$, and similarly, let $\bar{N}_{2}$ denote the blowup of $N_{2}$.

Lemma 6.7 The cohomology $\widehat{H I}_{(k)}^{*}(X)$ of the complex $\widehat{\Omega I}_{(k)}^{*}(X)$ is isomorphic to $H I_{(k)}^{*}(X)$.

Proof The inclusion of complexes

$$
\Omega I_{(k)}^{*}(X) \hookrightarrow \widehat{\Omega I}_{(k)}^{*}(X),
$$

described above induces a map on cohomology. We need to show this map is a bijection. We start with injectivity. Assume that $[\alpha] \in H I_{(k)}^{j}(X)$ and that $\alpha=d \hat{\beta}$ for $\hat{\beta} \in \widehat{\Omega I}_{(k)}^{j-1}(X)$. The two complexes differ only by the structure of forms on $\bar{N}$. To prove injectivity, we will thus only need to modify forms in a small neighborhood of $\bar{N}$. We will use $\bar{N}_{2}$ as this neighborhood.

When we restrict $\hat{\beta}$ to $\bar{N}_{2}$, we have

$$
\widehat{\beta}(x)=\widehat{\beta}_{t}(x)+d x \wedge \hat{\beta}_{n}(x),
$$


where $x$ is the coordinate in $[0,2)$, and where the $\hat{\beta}_{t / n}(x)$ are, for each fixed value of $x$, smooth forms on $Y=\partial \bar{M}$. Now on all of $\bar{M}$, define a new form

$$
\beta=\widehat{\beta}-d\left(\chi \int_{0}^{x} \hat{\beta}_{n}(t) d t\right),
$$

where $\chi$ is a smooth cutoff function on $\bar{M}$ which is identically 1 on $\bar{N}$ and is identically 0 on $\bar{M}-\bar{N}_{2}$ (we may in particular choose $\chi$ to be a pullback of a smooth cutoff on $[0,2)$ to $\bar{N}_{2}$ and extend it by zero to the rest of $\bar{M}$ ). Note that $\operatorname{inc}^{*}(\beta)=\operatorname{inc}^{*}(\hat{\beta})$, so $\beta \in \widehat{\Omega I}_{(k)}^{j-1}(X)$. But additionally, $\left.d x\right\lrcorner \beta=0$ on $\bar{N}$, because $\beta=\left(\widehat{\beta}_{t}(x)-\int_{0}^{x} d_{Y} \widehat{\beta}_{n}(t) d t\right)+d x \wedge 0$.

Since, on $\bar{N}, \alpha=\pi_{Y}^{*} \eta$ for some $\eta \in \Omega_{\mathcal{M S}}^{j}(\Sigma)$ and $d \beta=d \hat{\beta}=\alpha$, we have on $\bar{N}$ that

$$
\pi_{Y}^{*} \eta=d \beta(x)=d_{Y} \beta(x)+d x \wedge \beta^{\prime}(x),
$$

where $\beta^{\prime}(x)=(\partial / \partial x) \beta(x)$. Thus we must have $\beta^{\prime}(x)=0$, so $\beta(x)$ is constant in $x$ on $\bar{N}$. This means $\beta=\pi_{Y}^{*} \sigma$ for some $\sigma=$ inc $^{*} \beta=$ inc $^{*} \widehat{\beta} \in\left(\widehat{\mathrm{ft}}_{\geq k} \Omega^{*}(Y)\right)^{j-1}$. Since $d$ commutes with pullbacks, we have $d_{Y} \sigma=\eta$.

To complete the injectivity proof, we need to show now that we can further adapt $\hat{\beta}$ to a $\widetilde{\beta}=\pi_{Y}^{*} \widetilde{\sigma}$ where $\widetilde{\sigma}$ is multiplicatively structured and still in the right cotruncated complex. What we have done so far permits us to reduce this to showing that if $\eta=d_{Y} \sigma$, where $\sigma \in\left(\widehat{\mathrm{ft}}_{\geq k} \Omega^{*}(Y)\right)^{j-1}$ and $\eta \in\left(\mathrm{ft}_{\geq k} \Omega_{\mathcal{M S}}^{*}(\Sigma)\right)^{j}$, then $\eta=d_{Y} \widetilde{\sigma}$, where $\tilde{\sigma} \in\left(\mathrm{ft}_{\geq k} \Omega_{\mathcal{M S}}^{*}(\Sigma)\right)^{j-1}$.

As noted in Theorem 6.6, the injectivity of the Künneth isomorphism, $d_{Y} \sigma=\eta$, where $\eta \in \Omega_{\mathcal{M S}}^{j}(\Sigma)$, implies that $\sigma=d_{Y} \gamma+\sigma^{\prime}$, where $\sigma^{\prime} \in \Omega_{\mathcal{M S}}^{j-1}(\Sigma)$. Thus $d_{Y} \sigma^{\prime}=\eta$. We need to show that in addition, $\sigma^{\prime}$ can be chosen to satisfy the correct cotruncation. Expanding $d_{Y} \sigma^{\prime}=\eta$ in terms of bidegree, we get

$$
\begin{gathered}
\tilde{d}_{L} \sigma_{j-1,0}^{\prime}=\eta_{j, 0}, \\
\tilde{d}_{L} \sigma_{j-2,1}^{\prime}+(-1)^{j-1} \tilde{d}_{\Sigma} \sigma_{j-1,0}^{\prime}=\eta_{j-1,1}, \\
\vdots \\
\tilde{d}_{L} \sigma_{k-1, j-k}^{\prime}+(-1)^{k} \tilde{d}_{\Sigma} \sigma_{k, j-k-1}^{\prime}=\eta_{k, j-k}, \\
\tilde{d}_{L} \sigma_{k-2, j-k+1}^{\prime}+(-1)^{k-1} \tilde{d}_{\Sigma} \sigma_{k-1, j-k}^{\prime}=0,
\end{gathered}
$$


Decompose the term $\sigma_{k, j-1-k}^{\prime}$ according to the $L$ Hodge decomposition as

$$
\sigma_{k, j-1-k}^{\prime}=\tilde{d}_{L} a_{k-1, j-1-k}+\tilde{\delta}_{L} b_{k+1, j-1-k}+c_{k, j-1-k},
$$

and set

$$
\tilde{\sigma}=\sigma_{j-1,0}^{\prime}+\cdots+\sigma_{k+1, j-k-2}^{\prime}+\tilde{\delta}_{L} b_{k+1, j-1-k}+c_{k, j-1-k} .
$$

Note that in the $L$ Hodge decomposition of a multiplicatively structured form, each term is itself multiplicatively structured. This means that $\tilde{\sigma} \in\left(\mathrm{ft}_{\geq k} \Omega_{\mathcal{M S}}^{*}(\Sigma)\right)^{j-1}$. We would like to have that $d_{Y} \tilde{\sigma}=\eta$. When we decompose this equation by bidegree, we get exactly the same equations as in the previous decomposition for all equations above the one for $\eta_{k, j-k}$ (labelled (19)), so these are still correct. For the equations corresponding to those below (19), we get just $0+0=0$, which is also correct. So we just need to check that the equation corresponding to (19) is also correct:

$$
(-1)^{k} \tilde{d}_{\Sigma}\left(\tilde{\delta}_{L} b_{k+1, j-1-k}+c_{k, j-1-k}\right)=\eta_{k, j-k} .
$$

So consider again (19):

$$
\begin{array}{r}
\eta_{k, j-k}=\tilde{d}_{L} \sigma_{k-1, j-k}^{\prime}+(-1)^{k} \tilde{d}_{\Sigma}\left(\tilde{d}_{L} a_{k-1, j-1-k}+\tilde{\delta}_{L} b_{k+1, j-1-k}+c_{k, j-1-k}\right) \\
=\tilde{d}_{L}\left(\sigma_{k-1, j-k}^{\prime}+(-1)^{k} \tilde{d}_{\Sigma} a_{k-1, j-1-k}\right)+\tilde{\delta}_{L}(-1)^{k} \tilde{d}_{\Sigma} b_{k+1, j-1-k} \\
+(-1)^{k} \tilde{d}_{\Sigma} c_{k, j-1-k},
\end{array}
$$

where in the last step, we have used the fact that $\tilde{d}_{\Sigma}$ commutes up to sign with both $\tilde{d}_{L}$ and $\tilde{\delta}_{L}$. This is exactly the $L$ Hodge decomposition of $\eta_{k, j-k}$. However, the fact that $\tilde{\delta}_{L} \eta_{k, j-k}=0$ implies that the $\tilde{d}_{L}$ term in its decomposition vanishes, ie

$$
\tilde{d}_{L}\left(\sigma_{k-1, j-k}^{\prime}+(-1)^{k} \tilde{d}_{\Sigma} a_{k-1, j-1-k}\right)=0 .
$$

This means that (19) reduces to

$$
\eta_{k, j-k}=\tilde{\delta}_{L}\left((-1)^{k} \tilde{d}_{\Sigma} b_{k+1, j-1-k}\right)+(-1)^{k} \tilde{d}_{\Sigma} c_{k, j-1-k},
$$

which is equivalent to (20), as desired. This completes the injectivity part of the proof. Now consider surjectivity. Let $[\widehat{\beta}] \in \widehat{H I}_{(k)}^{j}(X)$. We need to construct a form $\widetilde{\beta} \in$ $\Omega I_{(k)}^{j}(X)$ such that $\hat{\beta}=\widetilde{\beta}+d \tilde{\eta}$ for some $\widetilde{\eta} \in \widehat{\Omega I}_{(k)}^{j-1}(X)$.

Restricting forms to $\bar{N}_{2}$, we again have

$$
\widehat{\beta}(x)=\hat{\beta}_{t}(x)+d x \wedge \hat{\beta}_{n}(x) .
$$


As before, on $\bar{M}$, define

$$
\beta=\hat{\beta}-d\left(\chi \int_{0}^{x} \hat{\beta}_{n}(t) d t\right) .
$$

By the same arguments as above, we get that $[\widehat{\beta}]=[\beta] \in \widehat{H I}_{(k)}^{j}(X)$, and on $\bar{N}$, $\beta=\pi_{Y}^{*} \sigma$ for some $\sigma \in\left(\widehat{\mathrm{ft}}_{\geq k} \Omega^{*}(Y)\right)^{j}$. We now need to adapt $\beta$ to a multiplicatively structured $\tilde{\beta}$. As before, this reduces to adapting the form $\sigma$ on $Y$ to an element of $\left(\mathrm{ft}_{\geq k} \Omega_{\mathcal{M S}}^{*}(\Sigma)\right)^{j}$.

First note that $d \hat{\beta}=0$ implies that $d_{Y} \sigma=0$. Thus using the Hodge decomposition on $Y$, we get

$$
\sigma=d_{Y} \eta+\sigma^{\prime}
$$

where $\sigma^{\prime}$ is harmonic in $Y$, and therefore multiplicatively structured as a sum of wedges of harmonic forms on $L$ and $\Sigma$. (This follows from the double Hodge decomposition Lemma 6.8 together with the Künneth theorem.) Now as in the injectivity argument, decomposing by bidegree and using the Hodge decomposition and the fact that $\sigma \in$ $\widehat{\mathrm{ft}}_{\geq k} \Omega^{j}(Y)$, we get that $\sigma^{\prime} \in\left(\mathrm{ft}_{\geq k} \Omega_{\mathcal{M S}}^{*}(\Sigma)\right)^{j}$ as desired.

We next need to show that we can choose $\eta \in\left(\widehat{\mathrm{ft}}_{\geq k} \Omega^{*}(Y)\right)^{j-1}$ in (21). Decomposing (21) by bidegree, we also get that $d_{Y}\left(\eta_{k-1, j-k}+\cdots+\eta_{0, j-1}\right)=0$. Thus we may choose our $\eta$ without loss of generality such that $\eta_{k-1, j-k}+\cdots+\eta_{0, j-1}=0$. Finally, we have that $\tilde{\delta}_{L} \tilde{d}_{\Sigma} \eta_{k, j-k-1}=\tilde{\delta}_{L}\left(\sigma_{k, j-k}-\sigma_{k, j-k}^{\prime}\right)=0$. Thus $\eta \in\left(\widehat{\mathrm{ft}}_{\geq k} \Omega^{*}(Y)\right)^{j-1}$ as desired.

Finally, we let

$$
\widetilde{\beta}=\beta-d(\chi \eta)=\widehat{\beta}-d\left(\chi\left(\eta+\int_{0}^{x} \widehat{\beta}_{n}(t) d t\right)\right)=\widehat{\beta}+d \tilde{\eta} .
$$

Since $[\tilde{\beta}]=[\widehat{\beta}] \in \widehat{H I}_{(k)}^{j}(X)$, where $[\widetilde{\beta}]$ is a class in $H I_{(k)}^{j}(X)$, the map is surjective.

When the link bundle is trivial, that is, $Y=L \times \Sigma$, we can analogously define operators $\tilde{d}_{\Sigma}$ and $\tilde{\delta}_{\Sigma}$. Then the Hodge decomposition theorem for forms on $Y$, Lemma 6.4, can be refined to a double Hodge decomposition.

Lemma 6.8 Let $\omega \in \Omega^{i, j}(L \times \Sigma)$. Then $\omega$ may be decomposed uniquely in a double Hodge decomposition as

$$
\begin{aligned}
\omega=\tilde{d}_{L} \tilde{d}_{\Sigma} a_{i-1, j-1}+ & \tilde{d}_{L} \tilde{\delta}_{\Sigma} a_{i-1, j+1}+\tilde{d}_{L} h_{i-1, j}^{\Sigma}+\tilde{\delta}_{L} \tilde{d}_{\Sigma} a_{i+1, j-1} \\
& +\tilde{\delta}_{L} \tilde{\delta}_{\Sigma} a_{i+1, j+1}+\tilde{\delta}_{L} h_{i+1, j}^{\Sigma}+\tilde{d}_{\Sigma} h_{i, j-1}^{L}+\tilde{\delta}_{\Sigma} h_{i, j+1}^{L}+h_{i, j}^{Y},
\end{aligned}
$$


where the terms $h_{*, j *}^{\Sigma}$ are in the kernels of $\tilde{d}_{\Sigma}$ and $\tilde{\delta}_{\Sigma}$, the terms $h_{*, *}^{L}$ are in the kernels of $\tilde{d}_{L}$ and $\tilde{\delta}_{L}$ and $h_{i, j}^{Y}$ is in the kernels of $d_{Y}$ and $\delta_{Y}$.

Proof This follows from the Hodge decompositions of each factor in

$$
\begin{aligned}
& \Omega^{i, j}(L \times \Sigma) \\
& =\Omega^{i}(L) \hat{\otimes} \Omega^{j}(\Sigma) \\
& =\left(d_{L} \Omega^{i-1}(L)+\delta_{L} \Omega^{i+1}(L)+\mathcal{H}^{i}(L)\right) \hat{\otimes}\left(d_{\Sigma} \Omega^{j-1}(\Sigma)+\delta_{\Sigma} \Omega^{j+1}(\Sigma)+\mathcal{H}^{j}(\Sigma)\right)
\end{aligned}
$$

together with the definitions of $\widetilde{d}_{L / \Sigma}$ and $\tilde{\delta}_{L / \Sigma}$, which imply that

$$
\tilde{d}_{L} \tilde{d}_{\Sigma}\left(\Omega^{i-1}(L) \hat{\otimes} \Omega^{j-1}(\Sigma)\right):=d_{L} \Omega^{i-1}(L) \hat{\otimes} d_{\Sigma} \Omega^{j-1}(\Sigma),
$$

and similar equalities for $\tilde{d}_{L} \tilde{\delta}_{\Sigma}, \tilde{\delta}_{L} \tilde{d}$ and $\tilde{\delta}_{L} \tilde{\delta}_{\Sigma}$. Note that the finite-dimensionality of the space of harmonic forms on $L$ and on $\Sigma$ means that for the terms in the expansion of the product that contain harmonic forms, we in fact have that $\hat{\otimes}$ is simply the same as $\otimes$, so the operators satisfy $d_{L / \Sigma}=\tilde{d}_{L / \Sigma}$ and $\delta_{L / \Sigma}=\tilde{\delta}_{L / \Sigma}$.

This double Hodge decomposition has similar properties to the standard single Hodge decomposition. For instance, if $\tilde{d}_{\Sigma} \omega=0$, then applying this to the double decomposition of $\omega$, we get that

$$
\tilde{d}_{\Sigma} \tilde{\delta}_{\Sigma}\left(\tilde{d}_{L} a_{i-1, j+1}+\tilde{\delta}_{L} a_{i+1, j+1}+h_{i, j+1}^{L}\right)=0 .
$$

Taking the inner product in $L^{2}(\Sigma)$ of this with $\tilde{d}_{L} a_{i-1, j+1}+\tilde{\delta}_{L} a_{i+1, j+1}+h_{i, j+1}^{L}$ at each point in $L$ implies that in fact

$$
\tilde{\delta}_{\Sigma}\left(\tilde{d}_{L} a_{i-1, j+1}+\tilde{\delta}_{L} a_{i+1, j+1}+h_{i, j+1}^{L}\right)=0 .
$$

\subsection{Proof of Theorem 1.3}

Theorem 1.3 follows from a sequence of lemmas relating the spaces $I H_{(q)}^{j}(\mathrm{CT}(X))$, $H I_{(j-q)}^{j}(X)$ and $I H_{(q+1)}^{j}(\mathrm{CT}(X))$. First we have the following lemma, which shows that there is a sequence of maps in each degree $j$ :

Lemma 6.9 For all $j$, there are well-defined maps

$$
I H_{(q)}^{j}(\mathrm{CT}(X)) \stackrel{A}{\longrightarrow} H I_{(j-q)}^{j}(X) \stackrel{B}{\longrightarrow} I H_{(q+1)}^{j}(\mathrm{CT}(X))
$$

that factorise the standard map $S_{q, q+1}$ between intersection cohomology groups of adjacent perversities. 
Proof First consider the map $A$. Let $\alpha \in I \Omega_{(q)}^{j}(\mathrm{CT}(X))$, with $d \alpha=0$. Then by definition of this complex, inc ${ }^{*} \alpha \in\left(\mathrm{ft}_{<q} \Omega^{*}(Y)\right)^{j}$. We can decompose inc ${ }^{*} \alpha$ by $(L, \Sigma)$ bidegree to get

$$
\operatorname{inc}^{*} \alpha=\alpha_{j, 0}+\cdots+\alpha_{j-q+1, q-1} .
$$

In particular, $\alpha_{0, j}+\cdots+\alpha_{j-q, q}=0$, which means that $\alpha \in \widehat{\Omega I}_{(j-q)}^{j}(X)$.

To show that this inclusion induces a well-defined map, $A$, on cohomology, we need to know that if $\alpha=d \eta$, where $\eta \in I \Omega_{(q)}^{j-1}(\mathrm{CT}(X))$, then we can find $\eta^{\prime} \in \widehat{\Omega I}_{(j-q)}^{j-1}(X)$ so that $\alpha=d \eta^{\prime}$, as well.

Decomposing by bidegree, we get

$$
\operatorname{inc}^{*} \eta=\eta_{j-1,0}+\cdots+\eta_{j-q, q-1} .
$$

Breaking down the equation inc ${ }^{*} \alpha=$ inc $^{*} d \eta=d_{Y}$ inc $^{*} \eta$ by bidegree, we get that $\tilde{d}_{\Sigma} \eta_{j-q, q-1}=0$. This means that in the double Hodge decomposition for $\eta_{j-q, q-1}$ we have

$$
\begin{aligned}
\eta_{j-q, q-1}=\tilde{d}_{L} \tilde{d}_{\Sigma} a_{j-q-1, q-2}+\tilde{\delta}_{L} \tilde{d}_{\Sigma} a_{j-q+1, q-2}+\tilde{d}_{\Sigma} h_{j-q, q-2}^{L}+\tilde{d}_{L} h_{j-q-1, q-1}^{\Sigma} \\
+\tilde{\delta}_{L} h_{j-q+1, q-1}^{\Sigma}+h_{j-q, q-1}^{Y} .
\end{aligned}
$$

Note that $\tilde{d}_{\Sigma}\left(\tilde{d}_{\Sigma} a_{j-q-1, q-2}+h_{j-q-1, q-1}^{\Sigma}\right)=0$. For $\chi$ a smooth cutoff supported at the end of $M$, let $\eta^{\prime}=\eta-d\left(\chi\left(\tilde{d}_{\Sigma} a_{j-q-1, q-2}+h_{j-q-1, q-1}^{\Sigma}\right)\right)$. Then $d \eta^{\prime}=\alpha$ still, and inc* $\eta^{\prime}=\operatorname{inc}^{*} \eta-d_{Y}\left(\tilde{d}_{\Sigma} a_{j-q-1, q-2}+h_{j-q+1, q-1}^{\Sigma}\right)$

$$
=\eta_{j-1,0}+\cdots+\eta_{j-q+1, q-2}+\left(\eta_{j-q, q-1}-\tilde{d}_{L}\left(\tilde{d}_{\Sigma} a_{j-q-1, q-2}+h_{j-q-1, q-1}^{\Sigma}\right)\right) .
$$

So $\tilde{\delta}_{L} \eta_{j-q, q-1}^{\prime}=0$, so $\eta^{\prime} \in \widehat{\Omega I}_{(j-q)}^{j-1}(X)$, and the map $A$ is well defined.

Now consider the map $B$. Suppose that $\beta \in \widehat{\Omega I}_{(j-q)}^{j}(X)$ and $d \beta=0$. Then

$$
\text { inc }^{*} \beta=\beta_{j, 0}+\cdots+\beta_{j-q, q}, \quad \tilde{\delta}_{L} \beta_{j-q, q}=0,
$$

and so decomposing inc* $d \beta$ by bidegree, we have

$$
\begin{aligned}
0 & =\operatorname{inc}^{*} d \beta=d_{Y} \text { inc }^{*} \beta \\
& =\left(\tilde{d}_{L} \beta_{j, 0}\right)+\left(\tilde{d}_{L} \beta_{j-1,1}+(-1)^{j} \tilde{d}_{\Sigma} \beta_{j, 0}\right)+\cdots \pm\left(\tilde{d}_{\Sigma} \beta_{j-q, q}\right) .
\end{aligned}
$$

Thus $\tilde{d}_{\Sigma} \beta_{j-q, q}=0$, so $\beta \in I \Omega_{(q+1)}^{j}(\mathrm{CT}(X))$. We need to show the map induced by inclusion is well defined on cohomology. Assume that $\beta=d \mu$ for $\mu \in \widehat{\Omega I}_{(j-q)}^{j-1}(X)$; then decomposing by bidegree again, we have by definition of $\widehat{\Omega I}_{(j-q)}^{j-1}(X)$ that

$$
\text { inc }^{*} \mu=\mu_{j-1,0}+\cdots+\mu_{j-q, q-1}, \quad \tilde{\delta}_{L} \mu_{j-q, q-1}=0 .
$$


In particular, $\mu_{j-q-1, q}=0$, so $\mu \in I \Omega_{(q+1)}^{j-1}(\mathrm{CT}(X))$, so $B$ is well defined.

Finally, since on the form level, $A$ and $B$ are both given by inclusion of a closed form in the domain complex into the range complex, their composition factorises the natural $\operatorname{map} S_{q, q+1}$.

Next we have three lemmas showing $A$ is injective, $B$ is surjective and $\operatorname{ker}(B) \subset \operatorname{im}(A)$. Together, these prove Theorem 1.3.

Lemma 6.10 The map $A$ is injective.

Proof Suppose that $A[\alpha]=0$, that is, $\alpha \in I \Omega_{(q)}^{j}(\mathrm{CT}(X)), d \alpha=0$ and $\alpha=d \beta$ for $\beta \in \widehat{\Omega I}_{(j-q)}^{j-1}(X)$. Then decomposing by bidegree,

$$
\text { inc }^{*} \beta=\beta_{j-1,0}+\cdots+\beta_{j-q, q-1}, \quad \tilde{\delta}_{L} \beta_{j-q, q-1}=0 .
$$

Because the degree in $\Sigma$ is $\leq q-1$ for all pieces, inc $\beta \in\left(\mathrm{ft}_{<q} \Omega^{*}(Y)\right)^{j-1}$. Also, by hypothesis, $d \beta=\alpha$ where inc ${ }^{*} \alpha \in\left(\mathrm{ft}_{<q} \Omega^{*}(Y)\right)^{j}$, so $\beta \in I \Omega_{(q)}^{j-1}(\mathrm{CT}(X))$, and $0=[\alpha] \in I H_{(q)}^{j}(\mathrm{CT}(X))$. Thus $A$ is injective.

Lemma 6.11 The map $B$ is surjective.

Proof Suppose that $[\gamma] \in I H_{(q+1)}^{j}(\mathrm{CT}(X))$. Then decomposing by bidegree, we have

$$
\text { inc }^{*} \gamma=\gamma_{j, 0}+\cdots+\gamma_{j-q, q} \text {. }
$$

Since $d \gamma=0$, we get that $\tilde{d}_{\Sigma} \gamma_{j-q, q}=0$. Decompose $\gamma_{j-q, q}$ according to the double Hodge decomposition:

$$
\begin{aligned}
\gamma_{j-q, q}=\tilde{d}_{L} \tilde{d}_{\Sigma} a_{j-q-1, q-1}+\tilde{\delta}_{L} \tilde{d}_{\Sigma} a_{j-q+1, q-1}+\tilde{d}_{\Sigma} h_{j-q, q-1}^{L}+\tilde{d}_{L} h_{j-q-1, q}^{\Sigma} \\
+\tilde{\delta}_{L} h_{j-q+1, q}^{\Sigma}+h_{j-q, q}^{Y}
\end{aligned}
$$

Note that $\tilde{d}_{\Sigma}\left(\tilde{d}_{\Sigma} a_{j-q-1, q-1}+h_{j-q-1, q}^{\Sigma}\right)=0$. Then for $\chi$ a smooth cutoff supported at the end of $M$, let

$$
\gamma^{\prime}=\gamma-d\left(\chi\left(\tilde{d}_{\Sigma} a_{j-q-1, q-1}+h_{j-q-1, q}^{\Sigma}\right)\right)
$$

Then we have $d \gamma^{\prime}=0$ and

$$
\text { inc }^{*} \gamma^{\prime}=\gamma_{j, 0}+\cdots+\left(\gamma_{j-q, q}-\tilde{d}_{L}\left(\tilde{d}_{\Sigma} a_{j-q-1, q-1}+h_{j-q-1, q}^{\Sigma}\right)\right),
$$

so $\tilde{\delta}_{L} \gamma_{j-q, q}^{\prime}=0$. Thus $\gamma^{\prime} \in \widehat{\Omega I}_{(j-q)}^{j}(X)$ represents a class in $H I_{(j-q)}^{j}(X)$. 
Further,

$$
\begin{aligned}
& \operatorname{inc}^{*}\left(\chi\left(\tilde{d}_{\Sigma} a_{j-q-1, q-1}+h_{j-q-1, q}^{\Sigma}\right)\right. \\
&=\tilde{d}_{\Sigma} a_{j-q-1, q-1}+h_{j-q-1, q}^{\Sigma} \in\left(\mathrm{ft}_{<q+1} \Omega^{*}(Y)\right)^{j-1},
\end{aligned}
$$

so $\left[\gamma^{\prime}\right]=[\gamma] \in I H_{(q+1)}^{j}(\mathrm{CT}(X))$. Thus $[\gamma]=B\left[\gamma^{\prime}\right]$, so $B$ is surjective.

Lemma 6.12 The kernel of $B$ is contained in the image of $A$.

Proof Assume that $B[\beta]=0$, that is, $\beta \in \widehat{\Omega I}_{(j-q)}^{j}(X)$ and $\beta=d \gamma$ for $\gamma \in$ $I \Omega_{(q+1)}^{j-1}(\mathrm{CT}(X))$. Then decomposing inc ${ }^{*} \beta$ by bidegree as in (22) and using the fact that $d \beta=0$, we get that $\tilde{d}_{\Sigma} \beta_{j-q, q}=0=\tilde{\delta}_{L} \beta_{j-q, q}$.

Now decomposing inc ${ }^{*} \gamma$ and inc ${ }^{*} d \gamma$ by bidegree, we get that

$$
\begin{aligned}
\text { inc }^{*} \gamma= & \gamma_{j-1,0}+\cdots+\gamma_{j-1-q, q}, \\
\text { inc }^{*} d \gamma= & \left(\tilde{d}_{L} \gamma_{j-1,0}\right)+\left((-1)^{j-1} \tilde{d}_{\Sigma} \gamma_{j-1,0}+\tilde{d}_{L} \gamma_{j-2,1}\right) \\
& \quad+\cdots+\left((-1)^{j-q} \tilde{d}_{\Sigma} \gamma_{j-q, q-1}+\tilde{d}_{L} \gamma_{j-1-q, q}\right)+\left((-1)^{j-q-1} \tilde{d}_{\Sigma} \gamma_{j-1-q, q}\right) .
\end{aligned}
$$

Thus $\tilde{d}_{\Sigma} \gamma_{j-1-q, q}=0$ and

$$
(-1)^{j-q} \tilde{d}_{\Sigma} \gamma_{j-q, q-1}+\tilde{d}_{L} \gamma_{j-1-q, q}=\beta_{j-q, q} .
$$

Decompose $\gamma_{j-q, q-1}$ by the Hodge decomposition in $L$ :

$$
\gamma_{j-q, q-1}=\tilde{d}_{L} a_{j-q-1, q-1}+\tilde{\delta}_{L} b_{j-q+1, q-1}+c_{j-q, q-1} .
$$

Then recalling that $\tilde{\delta}_{L} \beta_{j-q, q}=0$ and applying the Hodge decomposition in $L$ to all of (23), we get

$$
\beta_{j-q, q}=(-1)^{j-q} \tilde{d}_{\Sigma}\left(\tilde{\delta}_{L} b_{j-q+1, q-1}+c_{j-q, q-1}\right) .
$$

Let

$$
\beta^{\prime}:=\beta-d \chi\left(\tilde{\delta}_{L} b_{j-q+1, q-1}+c_{j-q, q-1}\right) .
$$

Note $\left(\widetilde{\delta}_{L} b_{j-q+1, q-1}+c_{j-q, q-1}\right) \in \widehat{\mathrm{ft}}_{\geq j-q} \Omega^{*}(Y)$, so $\left[\beta^{\prime}\right]=[\beta] \in H I_{(j-q)}^{j}(X)$. But

$$
\begin{aligned}
\text { inc }^{*} \beta^{\prime} & =\operatorname{inc}^{*} \beta-d_{Y}\left(\tilde{\delta}_{L} b_{j-q+1, q-1}+c_{j-q, q-1}\right) \\
& =\beta_{j, 0}+\cdots+\beta_{j-q+1, q-1}-\tilde{d}_{L} \tilde{\delta}_{L} b_{j-q+1, q-1},
\end{aligned}
$$

so $\beta^{\prime} \in I \Omega_{(q)}^{j}(\mathrm{CT}(X))$. Thus $[\beta]=A\left[\beta^{\prime}\right]$.

To put these lemmas together, we will use the following general result: 
Lemma 6.13 Assume that $X, Y$ and $Z$ are vector spaces and we have an injection $A: X \rightarrow Y$ and surjection $B: Y \rightarrow Z$ such that the kernel of $B$ is contained in the image of $A$. Then

$$
Y \cong X \oplus \frac{Z}{\operatorname{im}(B \circ A)}
$$

Proof Choose a splitting $Y=\operatorname{im}(A) \oplus W$ and a further splitting $\operatorname{im}(A)=\operatorname{ker}(B) \oplus V$. Then $Y=\operatorname{ker}(B) \oplus V \oplus W$. Note that $A$ is an isomorphism $X \cong \operatorname{im}(A)=\operatorname{ker}(B) \oplus V$ and $B$ is an isomorphism $V \oplus W \cong Z$. Further,

$$
(B \circ A)(X)=B(\operatorname{im}(A))=B(\operatorname{ker}(B) \oplus V)=B(V) \cong V .
$$

Thus $W \cong Z / \operatorname{im}(B \circ A)$. Thus

$$
Y=\operatorname{ker}(B) \oplus V \oplus W \cong X \oplus \frac{Z}{\operatorname{im}(B \circ A)} .
$$

Finally, we can complete the proof of Theorem 1.3 by applying this lemma to the maps

We get

$$
I H_{(q)}^{j}(\mathrm{CT}(X)) \stackrel{A}{\longrightarrow} H I_{(j-q)}^{j}(X) \stackrel{B}{\longrightarrow} I H_{(q+1)}^{j}(\mathrm{CT}(X)) .
$$

$$
\begin{aligned}
H I_{(j-q)}^{j}(X) & \cong I H_{(q)}^{j}(\mathrm{CT}(X)) \oplus \frac{I H_{(q+1)}^{j}(\mathrm{CT}(X))}{\operatorname{im}(B \circ A)} \\
& =I H_{(q)}^{j}(\mathrm{CT}(X)) \oplus \frac{I H_{(q+1)}^{j}(\mathrm{CT}(X))}{\operatorname{im}\left(S_{q, q+1}\right)} \\
& =: I G_{(q+1)}^{j}(\mathrm{CT}(X)) .
\end{aligned}
$$

To reindex, define $\bar{p}$ such that

$$
q+1=j+1-k:=j-1-(l-\bar{p}(l+1)) .
$$

Then $\bar{p}(l+1)=q-j+l$, so

$$
H I_{d R, \bar{p}}^{j}(X):=H I_{(l-\bar{p}(l+1))}^{j}(X)=H I_{(l-(q-j+l))}^{j}(X)=H I_{(j-q)}^{j}(X) .
$$

Thus $H I_{d R, \bar{p}}^{j}(X) \cong I G_{(j+i-k)}^{j}(\mathrm{CT}(X))$, as desired.

\section{The Hodge theorem for $\mathrm{HI}$}

Our Hodge theorem relates to the spaces of extended weighted $L^{2}$ harmonic forms over $M=X-\Sigma$ with respect to the various metrics we consider. A weighted $L^{2}$ 
space for any metric $g$ on $M$ is a space of forms:

$$
x^{c} L_{g}^{2} \Omega_{g}^{*}(M):=\left\{\omega \in \Omega^{*}(M) \mid \int_{M}\left\|x^{-c} \omega\right\|_{g}^{2} \operatorname{dvol}_{g}<\infty\right\} .
$$

Here $\|\cdot\|_{g}$ is the pointwise metric on the space of differential forms over $M$ induced by the metric on $M$ and $x$ is the function on $M$ that extends the $(0,1)$ coordinate on the end of $M$. The space $x^{c} L_{g}^{2} \Omega_{g}^{*}(M)$ can be completed to a Hilbert space with respect to the inner product (see standard references, eg [28, Theorem 3.2-3.3])

$$
\langle\alpha, \beta\rangle_{c}:=\int_{M} \alpha \wedge x^{-2 c} *_{g} \beta .
$$

Let $d$ represent the de Rham differential on smooth forms over $M$ and $\delta_{g, c}$ represent its formal adjoint with respect to the $x^{c} L^{2}$ inner product induced by the metric $g$. Then $D_{g, c}:=d+\delta_{g, c}$ is an elliptic differential operator on the space of smooth forms over $M$. If $c=0$, the elements of the kernel of $D_{g, 0}$ that lie in $L^{2}$ are the standard space of $L^{2}$ harmonic forms over $(M, g)$. More generally, we write

$$
\mathcal{H}_{L^{2}}^{j}(M, g, c):=\left\{\omega \in x^{c} L_{g}^{2} \Omega_{g}^{j}(M) \mid D_{g, c} \omega=0\right\} .
$$

Definition 7.1 The space of extended $x^{c} L^{2}$ harmonic forms on $(M, g)$ is

$$
\mathcal{H}_{\mathrm{ext}}^{*}(M, g, c):=\bigcap_{\epsilon>0}\left\{\omega \mid \omega \in x^{c-\epsilon} L_{g}^{2} \Omega_{g}^{*}(M), D_{g, c} \omega=0\right\} .
$$

\subsection{Proof of Theorem 1.1}

The space $I G_{(q)}^{j}(W)$ arises in extended Hodge theory for manifolds with fibred cusp metrics, studied by the second author in [25], and this allows us to prove Theorem 1.1. In [25], the second author considered the situation in which the regular stratum $M$ of a pseudomanifold $X$ with a single singular stratum $B$ is endowed with a fibred cusp metric. It is important to note that when we apply the results of that paper here, we are applying them to the space $\mathrm{CT}(X)$ rather than the space $X$ considered in the current paper. Thus, although the manifold $M$, which is the regular stratum of both $X$ and $\mathrm{CT}(X)$, is the same as the one considered in [25], the role of the base and fibre of $\partial M=Y=L \times \Sigma$ will switch depending on if we are considering $M$ as the regular stratum of $X$ or of CT $(X)$. In particular, the base of $M$ in [25], referred to as $B$ in that paper, corresponds to the fibre $L$ considered in the current paper. Correspondingly, the fibre $F$ considered in [25] corresponds to the base $\Sigma$ in the current paper. With this notation in mind, Theorem 1.2 from [25] may be rephrased in this situation as: 
Theorem 7.2 [25] Let $\left(M, g_{\mathrm{fc}}\right)$ be the regular stratum of $\mathrm{CT}(X)$ and $x$ be a smooth positive function on $M$ restricting to the radial coordinate at the end, as defined before. Endow $M$ with a geometrically flat fibred cusp metric, $g_{\text {fc }}$, for the fibration $\phi: L \times \Sigma \rightarrow L$. Then

where $b=\operatorname{dim} \Sigma$.

$$
\mathcal{H}_{\mathrm{ext}}^{j}\left(M, g_{\mathrm{fc}}, c\right) \cong I G_{((b / 2)+1-c)}^{j}(\mathrm{CT}(X)),
$$

Corollary 7.3 Under the conditions of Theorem 7.2, if $g_{\mathrm{fb}}=x^{-2} g_{\mathrm{fc}}$ is the fibred boundary metric conformal to $g_{\mathrm{fc}}$, then

$$
\mathcal{H}_{\mathrm{ext}}^{j}\left(M, g_{\mathrm{fb}}, c\right)=\mathcal{H}_{\mathrm{ext}}^{j}\left(M, g_{\mathrm{fc}}, c+\frac{1}{2} n-j\right) \cong I G_{(q)}^{j}(\mathrm{CT}(X)),
$$

where $q=j-\frac{1}{2}(b-1)-c$ and $n=\operatorname{dim}(M)=l+b+1$.

Proof The proof of this corollary is not difficult, but is somewhat long and technical in that it uses the spaces of $\mathrm{fc}-$ and $\mathrm{fb}-$ forms on $M$. The basic idea is that if we take $g_{\mathrm{fb}}$ to be the conformally related fibred boundary metric on $M$, then the conformal relationship $g_{\mathrm{fb}}=x^{-2} g_{\mathrm{fc}}$ means that

$$
x^{c} L_{\mathrm{fb}}^{2} \Omega_{\mathrm{fb}}^{j}(M)=x^{c+n / 2-j} L_{\mathrm{fc}}^{2} \Omega_{\mathrm{fc}}^{j}(M) .
$$

This means for the Hodge star operators that also $*_{\mathrm{fb}, c}=*_{\mathrm{fc}, c+(n / 2)-j}$, so in fact the extended harmonic forms in these spaces are the same. For more details, see the definitions of fc- and fb-forms and similar calculations to the above in [23].

Note that changing the role of fibre and base in the boundary fibration on $M$ means that the fibred boundary metric $g_{\mathrm{fb}}$ on $M$ with respect to the fibration $L \times \Sigma \rightarrow L$ can also be thought of as a fibred scattering metric $g_{\mathrm{fs}}$ on $M$ with respect to the fibration $L \times \Sigma \rightarrow \Sigma$. Thus we can rewrite this corollary as follows.

Corollary 7.4 Under the conditions of Theorem 7.2, if $\left(M, g_{\mathrm{fs}}\right)=\left(M, g_{\mathrm{fb}}\right)$ is the corresponding fibred scattering metric on $M$, then

$$
\mathcal{H}_{\mathrm{ext}}^{j}\left(M, g_{\mathrm{fs}}, c\right) \cong I G_{(q)}^{j}(\mathrm{CT}(X)),
$$

where $q=j-\frac{1}{2}(l-1)-c$.

Finally, if we set $q=j+1-(l-\bar{p}(l+1))$ as in Theorem 1.1, then this implies that $c=\frac{1}{2}(l-1)-\bar{p}(l+1)$ as claimed. 


\subsection{Example}

We consider the same space $X=S^{2} \times T^{2}$ as in Section 5.1, stratified as before. Then $M \cong \mathbb{R} \times S^{1} \times T^{2}$. We can endow this with a geometrically flat fibred scattering metric:

$$
g_{\mathrm{fs}}=d r^{2}+\left(1+r^{2}\right) d \theta_{1}^{2}+d \theta_{2}^{2}+d \theta_{3}^{2} .
$$

Note that if we make the change of coordinates $x=|r|^{-1}$ near $\pm \infty$, we get a metric that is a perturbation of one of the form in Definition 2.1 that decays like $x^{2}$. This turns out to be sufficient to use the same analysis (see [22]). We use this coordinate $r$ instead of $x$ because it makes the explicit calculations below easier to understand. In fact, in general, it is impossible to explicitly calculate the space of harmonic forms, and only the very simple structure of the space and metric here makes it possible.

If we consider extended $L^{2}$ harmonic forms on $\left(M, g_{\mathrm{fs}}\right)$ with no weight $(c=0)$, then Theorem 1.1 says

$$
\mathcal{H}_{\mathrm{ext}}^{*}\left(M, g_{\mathrm{fs}}, 0\right) \cong H I_{\mathrm{dR}, \bar{p}}^{*}(X),
$$

where $\frac{1}{2}(1-1)-\bar{p}(2)=0$. That is, the spaces of extended unweighted $L^{2}$ harmonic forms on $M$ should be isomorphic to the spaces with $\bar{p}(2)=0$ as we calculated in Section 5.1.

In order to identify the extended $L^{2}$ harmonic forms on $\left(M, g_{\mathrm{fs}}\right)$ it is useful to observe a few things. First, since the metric is a global product metric, the extended $L^{2}$ harmonic forms on $M$ are all products of extended $L^{2}$ harmonic forms on $W=\mathbb{R} \times S^{1}$ with harmonic forms on $T^{2}$. Thus it suffices to determine the extended harmonic forms on $W$ with the metric $g_{W}:=d r^{2}+\left(1+r^{2}\right) d \theta_{1}^{2}$.

Second, we observe that $g_{W}$ is a scattering metric, and is thus conformally invariant (with conformal factor $\left(1+r^{2}\right)$ ) to a b-metric. By the same argument as in Corollary 7.3, this means that extended harmonic forms on $\left(W, g_{W}\right)$ are the same as extended weighted $L^{2}$ harmonic forms on $\left(W,\left(1+r^{2}\right)^{-1} g_{W}\right)$. These forms are, in turn, known to be in the kernel of $d$ and $\delta$ independently (see either Proposition 6.16 in [30] or Lemma 4.3 in [25]). Thus we know that extended harmonic $L^{2}$-forms on $W$ are both closed and coclosed. This means that the only possible 0 -forms are constants and the only possible $2-$ forms are constant multiples of the volume form.

Third, recall that for a differential form to be extended harmonic, it must be in $x^{-\epsilon} L^{2} \Omega^{*}\left(W, g_{W}\right)$ for all $\epsilon>0$, or equivalently, $\left(1+r^{2}\right)^{-\epsilon} w \in L^{2} \Omega^{*}\left(W, g_{W}\right)$. 
If we consider constant functions, this means we need

$$
\int_{-\infty}^{\infty} c^{2}\left(1+r^{2}\right)^{1-2 \epsilon} d r<0
$$

This is not true, so $\mathcal{H}_{\mathrm{ext}}^{0}\left(W, g_{W}\right)=\{0\}$. By an analogous argument (or equivalently, by Poincaré duality), also $\mathcal{H}_{\text {ext }}^{2}\left(W, g_{W}\right)=\{0\}$.

Finally consider $\mathcal{H}_{\mathrm{ext}}^{1}\left(W, g_{W}\right)$. The space of extended $L^{2}$ harmonic forms of middle degree is preserved by a conformal change of metric, and as noted before, $g_{W}$ is conformally equivalent to the metric

$$
g_{b}=\frac{d r^{2}}{1+r^{2}}+d \theta_{1}^{2} .
$$

If we reparametrize, setting $t=\operatorname{arcsinh} r$, this becomes the metric on the infinite cylinder:

$$
g_{b}=d t^{2}+d \theta_{1}^{2}
$$

If we use a Fourier series decomposition in $\theta_{1}$, we find that a 1 -form

$$
\begin{aligned}
\omega=\eta_{0}(r) d r+\left(\sum_{n=0}^{\infty}\left(\eta_{1, n}(r) \cos \left(n \theta_{1}\right)+\eta_{2, n}(r) \sin \left(n \theta_{1}\right)\right) d r\right)+\mu_{0}(r) d \theta \\
+\sum_{n=0}^{\infty}\left(\mu_{1, n}(r) \cos \left(n \theta_{1}\right)+\mu_{2, n}(r) \sin \left(n \theta_{1}\right)\right) d \theta_{1}
\end{aligned}
$$

is closed and coclosed if $\eta_{0}(r)$ and $\mu_{0}(r)$ are constant and the remaining coefficients satisfy $f^{\prime \prime}=n^{2} f$, that is, they are all exponential functions in $t$, and thus blow up at either $\infty$ or $-\infty$, so are not almost in $L^{2}$. So the only extended $L^{2}$ harmonic forms are $c_{1} d \theta_{1}+c_{2} d t$, which are in $\left(1+t^{2}\right)^{\epsilon} L^{2} \Omega^{*}\left(W, g_{W}\right)$, as required. Thus $\mathcal{H}_{\text {ext }}^{1}\left(W, g_{W}\right) \cong \mathbb{R}^{2}$. Now when we take the tensor product with $\mathcal{H}^{*}\left(T^{2}\right)$, we get

$$
\begin{aligned}
& \mathcal{H}_{\mathrm{ext}}^{0}\left(M, g_{\mathrm{fs}}\right) \cong 0 \cong H I_{\mathrm{dR}, \bar{p}}^{0}(X), \\
& \mathcal{H}_{\mathrm{ext}}^{1}\left(M, g_{\mathrm{fs}}\right) \cong \mathbb{R}^{2} \cong H I_{\mathrm{dR}, \bar{p}}^{1}(X), \\
& \mathcal{H}_{\mathrm{ext}}^{2}\left(M, g_{\mathrm{fs}}\right) \cong \mathbb{R}^{4} \cong H I_{\mathrm{dR}, \bar{p}}^{2}(X), \\
& \mathcal{H}_{\mathrm{ext}}^{3}\left(M, g_{\mathrm{fs}}\right) \cong \mathbb{R}^{2} \cong H I_{\mathrm{dR}, \bar{p}}^{3}(X), \\
& \mathcal{H}_{\mathrm{ext}}^{4}\left(M, g_{\mathrm{fs}}\right) \cong 0 \cong H I_{\mathrm{dR}, \bar{p}}^{4}(X),
\end{aligned}
$$

as predicted by Theorem 1.1. 


\subsection{Explicit isomorphism for the Hodge theorem}

Although Theorem 1.1 shows that there exists an isomorphism from extended harmonic forms to $H I$ cohomology of $X$, the proof does not give an explicit description of a map that yields this isomorphism. Furthermore, although the spaces involved do not relate in any way to $\mathrm{CT}(X)$, the proof of the theorem critically uses relationships to $\mathrm{CT}(X)$. It would be nice if, as in the classical Hodge theorem, the isomorphism could be realized by sending an extended harmonic form to the $H I$ class that it represents: $\gamma \rightarrow[\gamma]$. That way, we obtain a map realising the isomorphism that makes sense without reference to $\mathrm{CT}(X)$. Furthermore, it gives us a place to start when trying to extend the $H I$ Hodge theorem to the case when the link bundle of $\Sigma$ in $X$ is twisted.

However, the extended harmonic forms in our Hodge theorem do not in general lie in either of the two complexes we have seen that calculate $H I_{\mathrm{dR}, \bar{p}}^{*}(X)$, as forms that are in the extended $L^{2}$ spaces over $M$ can in general have coefficients that blow up at the end of $M$. Thus to obtain an explicit map realising the Hodge isomorphism, that is to see extended harmonic forms as representatives of classes in the $H I$ space, we need new larger spaces of forms that can be used to calculate the $H I$ cohomology spaces and that do contain the extended harmonic forms. We can find spaces that work in this regard by reinterpreting the proof of Theorem 1.1. In this section, we find appropriate new spaces of forms by using the isomorphism with $I G$ and alternative complexes of forms that may be used to calculate $I H$. However, the spaces that we eventually obtain do not relate in any way to $\mathrm{CT}(X)$, and are defined entirely in terms of $X$ itself. In this sense they achieve our goal. Unfortunately, the spaces used in the definition do not fit into a cochain complex, so the necessary alternative description of the $H I$ cohomology spaces is not as topologically satisfying as it might be. Nevertheless, we believe that demonstrating an explicit isomorphism is useful.

From [25], we have the following setup and lemma which will allow us to see the extended harmonic forms as representing classes in $H I$. Assume that $W$ is a pseudomanifold with a single, smooth singular stratum $B$ of dimension $l$, whose link bundle with link $F$ of dimension $b$ is flat with respect to the structure group $\operatorname{Isom}\left(F, g_{F}\right)$ for some fixed metric on $F$. Let $M=W-B$ and let $x$ be a smooth function on $M$ that extends across $B$ in $W$ by zero. Let $\bar{M}$ be the complement in $W$ of a normal neighborhood of $B$, and let $i_{s}: \partial \bar{M} \rightarrow M$ denote the inclusion of $\partial \bar{M}$ into $M$ in the slice where $x=s$. 
Define the projection operator $\Pi_{q-1, q}$ on $\Omega^{*}(\partial \bar{M})$ by projection onto forms in fibre degree $q$ that lie in $\operatorname{ker}\left(\tilde{\delta}_{F}\right)$ and forms in fibre degree $q+1$ that lie in $\operatorname{im}\left(\tilde{d}_{F}\right)$ in terms of the $F$ Hodge decomposition. Let $x^{a} L^{2} \Omega_{\mathrm{con}}^{*}\left(M, g_{\mathrm{fc}}\right)$ denote the complex of forms on $M$ that are conormal at $x=0$ (see eg [30]), and are also in the $x^{a}$ weighted $L^{2}$ space on $M$ with respect to the metric $g_{\mathrm{fc}}$.

Lemma 7.5 The cohomology of the complex $x^{(b / 2)-q-\epsilon} L^{2} \Omega^{*}\left(M, g_{\text {fc }}\right.$ ) (made into a complex in the standard way by requiring both $\omega$ and $d \omega$ lie in the appropriate spaces) is isomorphic to $I H_{(q)}^{*}(W)$ and the cohomology of the complex

$$
\begin{aligned}
& x^{(b / 2)-q-\epsilon} L^{2} \Omega_{0}^{*}\left(M, g_{\mathrm{fc}}\right) \\
& :=\left\{\omega \in x^{(b / 2)-q-\epsilon} L^{2} \Omega_{\mathrm{con}}^{*}\left(M, g_{\mathrm{fc}}\right) \mid \lim _{s \rightarrow 0} \Pi_{q-1, q} \circ i_{s}^{*} \omega=0, \lim _{s \rightarrow 0} \Pi_{q-1, q} \circ i_{s}^{*} d \omega=0\right\}
\end{aligned}
$$

is isomorphic to $I H_{(q-1)}^{*}(W)$. Furthermore, using this definition of $I H_{(q-1)}^{*}(W)$, we have the following long exact sequence on cohomology:

$$
\rightarrow H^{j-q-1}\left(B ; H^{p}(F)\right) \stackrel{\delta}{\longrightarrow} I H_{(q-1)}^{j}(W) \stackrel{\text { inc }}{\longrightarrow} I H_{(q)}^{j}(W) \stackrel{r}{\longrightarrow} H^{j-q}\left(B ; H^{q}(F)\right) \rightarrow,
$$

where $r=\lim _{s \rightarrow 0} \Pi_{q-1, q} \circ i_{s}^{*}$.

These are the complexes used to prove Theorem 5.1 from [25], so from Corollary 7.3, letting $W=\mathrm{CT}(X), B=L$ and $\Sigma=F$, we have that the isomorphism in Corollary 7.4 is realized by an inclusion of the space of extended weighted $L^{2}$ harmonic forms into the numerator of the quotient space:

$$
\mathcal{H}_{\mathrm{ext}}^{j}\left(M^{\prime}, g_{\mathrm{fs}}, c\right) \rightarrow \frac{\operatorname{ker}(d) \subset x^{(b / 2)-q-\epsilon} L^{2} \Omega^{j}\left(M, g_{\mathrm{fc}}\right)}{d\left(x^{(b / 2)-q-\epsilon} L^{2} \Omega_{0}^{j}\left(M, g_{\mathrm{fc}}\right)\right)},
$$

where $q=j-\frac{1}{2}(l-1)-c$ for $l$ the dimension of $B$. We can reinterpret the spaces on the right in terms of the metric $g_{\text {fs }}$ to get

$$
\mathcal{H}_{\mathrm{ext}}^{j}\left(M^{\prime}, g_{\mathrm{fs}}, c\right) \rightarrow \frac{\operatorname{ker}(d) \subset x^{c-1-\epsilon} L^{2} \Omega^{j}\left(M^{\prime}, g_{\mathrm{fs}}\right)}{d\left(x^{c-1-\epsilon} L^{2} \Omega_{0}^{j}\left(M^{\prime}, g_{\mathrm{fs}}\right)\right)} .
$$

Using Theorem 1.3, we calculate $H I_{\mathrm{dR}, \bar{p}}^{j}(X)$ from this quotient:

$$
H I_{\mathrm{dR}, \bar{p}}^{j}(X) \cong \frac{\operatorname{ker}(d) \subset x^{(l-3) / 2-\bar{p}(l+1)-\epsilon} L^{2} \Omega^{*}\left(M^{\prime}, g_{\mathrm{fs}}\right)}{d\left(x^{(l-3) / 2-\bar{p}(l+1)-\epsilon} L^{2} \Omega_{0}^{*}\left(M^{\prime}, g_{\mathrm{fs}}\right)\right)} .
$$

This is then the definition of $H I_{\mathrm{dR}, \bar{p}}^{j}(X)$ for which the isomorphism in the Hodge theorem, Theorem 1.1, is given by the classical map $\gamma \rightarrow[\gamma]$. 


\section{Proof of Theorem 1.4}

In order to prove Theorem 1.4, we need to understand how the intersection pairing defined on the original de Rham cohomology of intersection spaces relates to the isomorphism in Theorem 1.3 and the intersection pairing on the de Rham intersection cohomology groups. First, we can show that the alternative complex we defined to calculate $H I_{\mathrm{dR}, \bar{p}}^{*}(X)$ also admits a natural intersection pairing by integration, and that this pairing is equivalent to the original pairing by the isomorphism in Lemma 6.7.

Lemma 8.1 Integration of wedge products defines a bilinear pairing between $\widehat{H I} \frac{j}{\bar{p}}(X)$ and $\widehat{H I}_{\bar{q}}^{n-j}(X)$ which is equal to the pairing by integration of wedge products between $H I_{\mathrm{dR}, \bar{p}}^{j}(X)$ and $H I_{\mathrm{dR}, \bar{q}}^{n-j}(X)$.

Proof First we show there is a well-defined bilinear pairing between $\widehat{H I}_{\bar{p}}^{j}(X)$ and $\widehat{H I}_{\bar{q}}^{n-j}(X)$. Let $\hat{\alpha} \in \widehat{\Omega I}_{\bar{p}}^{j}(X)$ and $\widehat{\beta} \in \widehat{\Omega I}_{\bar{q}}^{n-j}(X)$ be closed forms. Then $\int_{M} \widehat{\alpha} \wedge \widehat{\beta}$ is finite since both forms are smooth on $\bar{M}$. Furthermore, if $\hat{\alpha}=d \hat{\eta}$, where $\hat{\eta} \in$ $\widehat{\Omega I}_{\bar{p}}^{j-1}(X)$, then by Stokes's theorem,

$$
\int_{M} d \hat{\eta} \wedge \hat{\beta}=\int_{M} d(\hat{\eta} \wedge \hat{\beta})=\lim _{s \rightarrow 0} \int_{Y} \hat{\eta}(s) \wedge \hat{\beta}(s),
$$

where now $s$ is the coordinate on $(0,1)$ near the end, to correspond to the limits in Lemma 7.5. We can decompose $\hat{\eta}(s)$ and $\hat{\beta}(s)$ by $(L, \Sigma)$ bidegree. By definition of $\widehat{\Omega I}_{\bar{p}}^{j-1}(X)$ and $\widehat{\Omega I}_{\bar{q}}^{n-j}(X)$, and by the fact that $\bar{p}(l+1)+\bar{q}(l+1)=l-1$, we get

$$
\lim _{s \rightarrow 0} \sum_{i=0}^{k-1} \hat{\eta}_{i, j-1-i}(s)=0=\lim _{s \rightarrow 0} \sum_{i=0}^{l-k} \widehat{\beta}_{i,(n-j)-i}(s) .
$$

Thus the only part that can remain in the limit is

$$
=\lim _{s \rightarrow 0} \int_{Y}\left(\sum_{i=k}^{l} \hat{\eta}_{i, k-1-i}(s)\right) \wedge\left(\sum_{i=l+1-k}^{l} \hat{\beta}_{i, n-j-i}(s)\right) .
$$

But this also is zero, since none of the terms in the second sum is of complementary bidegree to any term in the first sum.

Because the inclusion map of forms from $\Omega I_{\bar{p}}^{j}(X) \hookrightarrow \widehat{\Omega I}_{\bar{p}}^{j}$ induces an isomorphism on cohomology for all $\bar{p}$ and $j$, and because the intersection pairing between classes in $\widehat{H I}_{\bar{p}}^{j}(X)$ and $\widehat{H I}_{\bar{q}}^{n-j}$ can be calculated from any representatives of these classes, the intersection pairing between these spaces is equal to the intersection pairing (also defined by integration) for $H I_{\bar{p}}^{j}(X)$ and $H I_{\bar{q}}^{n-j}$. 
Next, we want to trace this pairing through the proof of Theorem 1.3 to see how it can be interpreted in terms of the signature pairing on intersection cohomology on $\mathrm{CT}(X)$. Recall that we have

$$
H I_{\mathrm{dR}, \bar{p}}^{j}(X) \cong I G_{(t+1)}^{j}(\mathrm{CT}(X))=\frac{I H_{(t)}^{j}(\mathrm{CT}(X)) \oplus I H_{(t+1)}^{j}(\mathrm{CT}(X))}{\operatorname{im}\left(I H_{(t)}^{j}(\mathrm{CT}(X)) \rightarrow I H_{(t+1)}^{j}(\mathrm{CT}(X))\right)}
$$

where $t=j-l+\bar{p}(l+1)$. So also if $\bar{p}$ and $\bar{q}$ are dual perversities on $X$, then $\bar{p}(l+1)+\bar{q}(l+1)=l-1$ implies that

$$
H I_{\mathrm{dR}, \bar{q}}^{n-j}(X) \cong I G_{(s+1)}^{n-j}(\mathrm{CT}(X))=\frac{I H_{(s)}^{n-j}(\mathrm{CT}(X)) \oplus I H_{(s+1)}^{n-j}(\mathrm{CT}(X))}{\operatorname{im}\left(I H_{(s)}^{n-j}(\mathrm{CT}(X)) \rightarrow I H_{(s+1)}^{n-j}(\mathrm{CT}(X))\right)}
$$

where $s=n-j-1-\bar{p}(l+1)$. Observe that $t+s+1=n-l$, which is the codimension of the singular stratum in $\mathrm{CT}(X)$. This is the relationship we expect for the cutoff degrees for dual perversities in $I H_{*}^{*}(\mathrm{CT}(X))$. That is, the signature pairing for intersection cohomology on $\mathrm{CT}(X)$ pairs the first term in the top of (26) with the second term in the top of (27), and vice versa.

We can identify the right and left spaces in (26) in terms of the $H I$ space using the maps $A$ and $B$ from the proof of Theorem 1.3. To distinguish these maps in the two settings of (26) and (27), fix the notation

$$
I H_{(t)}^{j}(\mathrm{CT}(X)) \stackrel{A_{\bar{p}}}{\longrightarrow} H I_{\mathrm{dR}, \bar{p}}^{j}(X) \stackrel{B_{\bar{p}}}{\longrightarrow} I H_{(t+1)}^{j}(\mathrm{CT}(X))
$$

and similarly define $A_{\bar{q}}$ and $B_{\bar{q}}$ for the spaces in (27). Then we have

$$
I H_{(t)}^{j}(\mathrm{CT}(X)) \cong \operatorname{im}\left(A_{\bar{p}}\right) \quad \text { and } \quad I H_{(t+1)}^{j}(\mathrm{CT}(X)) \cong H I_{\mathrm{dR}, \bar{p}}^{j}(X) / \operatorname{ker}\left(B_{\bar{p}}\right),
$$

and analogous isomorphisms in the $\bar{q}$ case. Now we can precisely state the compatibility between the intersection pairing on $H I$ spaces and on $I H$ spaces.

Lemma 8.2 For $[\alpha] \in I H_{(t)}^{j}(\mathrm{CT}(X))$ and $[\beta] \in H I_{\mathrm{dR}, \bar{q}}^{n-j}(X)$,

$$
A_{\bar{p}}[\alpha] \cap_{H I}[\beta]=[\alpha] \cap_{I H} B_{\bar{q}}[\beta] .
$$

Proof Both of the pairings $\cap_{I H}$ and $\cap_{H I}$ are achieved on their corresponding de Rham cohomology spaces by integration of the wedge of representatives of the paired cohomology classes. Both are known to be well defined on their corresponding cohomologies. Further, by definition of the map $A_{\bar{p}}$, we can take the same representative form to represent both $[\alpha]$ and $A_{\bar{p}}[\alpha]$. Similarly, we can represent both $[\beta]$ and $B_{\bar{q}}[\beta]$ 
by the same form. Thus for $[\alpha]$ and $[\beta]$ as in the statement of the lemma,

$$
A_{\bar{p}}[\alpha] \cap_{H I}[\beta]:=\int_{M} \alpha \wedge \beta:=[\alpha] \cap_{I H} B_{\bar{q}}[\beta] .
$$

Note that this gives us the following corollary:

Corollary 8.3 The image $\operatorname{im}\left(A_{\bar{p}}\right)$ is the annihilator under the pairing $\cap_{H I}$ of $\operatorname{ker}\left(B_{\bar{q}}\right)$.

Proof Lemma 8.2 implies that if $[\beta] \in \operatorname{ker}\left(B_{\bar{q}}\right)$, then $A_{\bar{p}}[\alpha] \cap_{H I}[\beta]=[\alpha] \cap_{H I} 0=0$. So $\operatorname{im}\left(A_{\bar{p}}\right) \subset \operatorname{Ann}\left(\operatorname{ker}\left(B_{\bar{q}}\right)\right)$. So we just need to show this containment is an equality. Because the intersection pairing on $H I$ is nondegenerate, we know that

$$
\operatorname{dim}\left(\operatorname{ker}\left(B_{\bar{q}}\right)\right)=\operatorname{dim}\left(H I_{\bar{p}}^{j}(X) / \operatorname{Ann}\left(\operatorname{ker}\left(B_{\bar{q}}\right)\right)\right) .
$$

So we want to show that

$$
\operatorname{dim}\left(\operatorname{ker}\left(B_{\bar{q}}\right)\right)=\operatorname{dim}\left(H I_{\bar{p}}^{j}(X) / \operatorname{im}\left(A_{\bar{q}}\right)\right) .
$$

Recall that Poincaré duality for intersection cohomology gives isomorphisms

$$
I H_{(t)}^{n-j}(\mathrm{CT}(X)) \cong\left(I H_{(s+1)}^{j}(\mathrm{CT}(X))\right)^{*}:=\operatorname{im}\left(B_{\bar{p}} \circ A_{\bar{p}}\right)^{*} \oplus W^{*}
$$

for some complementary subspace $W$ and

$$
I H_{(t+1)}^{n-j}(\mathrm{CT}(X)) \cong\left(I H_{(s)}^{j}(\mathrm{CT}(X))\right)^{*}:=V^{*} \oplus \operatorname{ker}\left(B_{\bar{p}} \circ A_{\bar{p}}\right) *
$$

for a complementary subspace $V \cong \operatorname{im}\left(B_{\bar{p}} \circ A_{\bar{p}}\right)$. Suppose that we have classes $[\alpha]_{I H} \in I H_{(t)}^{j}(\mathrm{CT}(X))$ and $[\gamma] \in I H_{(s)}^{n-j}(\mathrm{CT}(X))$. Then by Lemma 8.2,

$$
A_{\bar{p}}[\alpha] \cap_{H I} A_{\bar{q}}[\gamma]=[\alpha] \cap_{I H} B_{\bar{q}} \circ A_{\bar{q}}[\gamma] .
$$

But applying Lemma 8.2 where we switch the roles of the perversities $\bar{q}$ and $\bar{p}$, we also have

$$
A_{\bar{p}}[\alpha] \cap_{H I} A_{\bar{q}}[\gamma]=B_{\bar{p}} \circ A_{\bar{p}}[\alpha] \cap_{I H}[\gamma] .
$$

Thus overall, we have $B_{\bar{p}} \circ A_{\bar{p}}[\alpha] \cap_{I H}[\gamma]=[\alpha] \cap_{I H} B_{\bar{q}} \circ A_{\bar{q}}[\gamma]$. Because Poincaré duality for intersection cohomology is realized by the intersection pairing between dual perversity and dual degree spaces, this means that under the duality map, also $B_{\bar{q}} \circ A_{\bar{q}}=\left(B_{\bar{p}} \circ A_{\bar{p}}\right) *$.

Thus $B_{\bar{q}} \circ A_{\bar{q}}$ must be an isomorphism from $\operatorname{im}\left(B_{\bar{p}} \circ A_{\bar{p}}\right)^{*}$ to $V^{*}$. This means that

$$
W^{*} \cong \operatorname{ker}\left(B_{\bar{q}} \circ A_{\bar{q}}\right) \cong \operatorname{ker}\left(B_{\bar{q}}\right),
$$

because $A_{\bar{q}}$ is an injection and $\operatorname{ker}\left(B_{\bar{q}}\right) \subset \operatorname{im}\left(A_{\bar{q}}\right)$. But also,

$$
W^{*} \cong W \cong H I_{\bar{p}}^{j}(X) / \operatorname{im}\left(A_{\bar{p}}\right) \text {. }
$$


Now let us focus on the setting where $X$ is an even-dimensional Witt space, so the map $S_{\bar{n}, \bar{m}}$ from the lower-middle perversity intersection cohomology to the upper-middle perversity intersection cohomology is an isomorphism. Note that this does not imply that $\mathrm{CT}(X)$ is Witt; for instance, $l$ could be odd and $H^{b / 2}(\Sigma) \neq 0$. We start with the decomposition

$$
H I_{\bar{m}}^{n / 2}(X)=\operatorname{ker}\left(B_{\bar{m}}\right) \oplus V \oplus W,
$$

where $I H_{\bar{n}}^{n / 2}(\mathrm{CT}(X)) \cong \operatorname{ker}\left(B_{\bar{m}}\right) \oplus V$ and $I H_{\bar{m}}^{n / 2}(\mathrm{CT}(X)) \cong V \oplus W$. Here $V=$ $\operatorname{im}\left(I H_{\bar{n}}^{n / 2}(\mathrm{CT}(X)) \rightarrow I H_{\bar{m}}^{n / 2}(\mathrm{CT}(X))\right)$, so the signature of the intersection pairing restricted to $V$ is the middle perversity perverse signature of $\mathrm{CT}(X)$. This is equal to the signature of the open manifold $M$ as well as the middle perversity signatures for $H I$ and $I H$ of the space $Z$ obtained as the one-point compactification of $M$. This follows from a result in [24], which calculates perverse $(I H)$ signatures for a pseudomanifold with a single smooth singular stratum as the sum of the signature on its complement (ie the signature of $M$ ) and a set of terms arising from the second and higher pages in the Leray spectral sequence of the link bundle of the singular stratum. In particular, if the spectral sequence degenerates at the second page, as it does in the case of a product bundle, all of these additional terms vanish, so all perverse signatures are simply the signature of $M$.

Thus we need to show that the signature of the intersection pairing restricted to each of the other two factors vanishes. For the factor $\operatorname{ker}\left(B_{\bar{m}}\right)$, this is true because it is contained in its annihilator, $\operatorname{im}\left(A_{\bar{m}}\right)$. For the factor $W$, we use the fact that under Poincaré duality applied to $I H_{\bar{m} / \bar{n}}^{n / 2}(X)$ the space $W$ is the dual of $\operatorname{ker}\left(B_{\bar{m}}\right)$. Because this map preserves intersection pairing, this means that the intersection pairing restricted to $W$ also vanishes.

It remains to show that $\sigma_{I H}(X)=\sigma_{I H}(Z)$. There are several ways to see this, for example as follows: By Siegel's pinch bordism (see [33] or [1, Chapter 6.6]), $\sigma_{I H}(X)=\sigma_{I H}(Z)+\sigma_{I H}(E)$, where $E$ is the pseudomanifold

$$
E=(c L) \times \Sigma \cup_{L \times \Sigma} c(L \times \Sigma) .
$$

If $l=\operatorname{dim} L$ is odd, then Lemma 8.1 of [6] implies that in fact already the group $I H_{n / 2}^{\bar{m}}(E)$ is trivial. In particular, $\sigma_{I H}(E)=0$ and $\sigma_{I H}(X)=\sigma_{I H}(Z)$. If $l$ is even, then $\operatorname{dim} \Sigma$ is odd and thus $\mathrm{CT}(X)$ is a Witt space. (Note that $\operatorname{dim} \Sigma$ odd means in particular that $\operatorname{dim} \Sigma \geq 1$ and thus that the singular set of $\operatorname{CT}(X)$ has codimension at least 2.) Hence we may apply what we have proved so far to $X^{\prime}=\mathrm{CT}(X)$ and 
obtain

$$
\sigma_{H I}(\mathrm{CT}(X))=\sigma_{I H, \bar{m}}(\mathrm{CT}(\mathrm{CT}(X)))=\sigma_{I H}(Z) .
$$

Since $\mathrm{CT}(\mathrm{CT}(X)) \cong X$ and $X$ is Witt, we have for the perverse signature

$$
\sigma_{I H, \bar{m}}(\mathrm{CT}(\mathrm{CT}(X)))=\sigma_{I H}(X) .
$$

Acknowledgements Banagl was in part supported by a research grant of the Deutsche Forschungsgemeinschaft. The authors thank the Deutsche Forschungsgemeinschaft and the London Mathematical Society for funding the research visits during which much of this work was done. Hunsicker thanks Timo Essig and Bryce Chriestenson for useful discussions. In particular, the proof of Lemma 6.3 is partly due to Essig and the proof of Lemma 6.1 is due to Chriestenson. Finally, both authors would like to thank the referee, whose very careful comments substantially improved the readability of the paper.

\section{References}

[1] M Banagl, Topological invariants of stratified spaces, Springer (2007) MR

[2] M Banagl, Intersection spaces, spatial homology truncation, and string theory, Lecture Notes in Mathematics 1997, Springer (2010) MR

[3] M Banagl, Isometric group actions and the cohomology of flat fiber bundles, Groups Geom. Dyn. 7 (2013) 293-321 MR

[4] M Banagl, Foliated stratified spaces and a de Rham complex describing intersection space cohomology, J. Differential Geom. 104 (2016) 1-58 MR

[5] M Banagl, N Budur, L Maxim, Intersection spaces, perverse sheaves and type IIB string theory, Adv. Theor. Math. Phys. 18 (2014) 363-399 MR

[6] M Banagl, S E Cappell, J L Shaneson, Computing twisted signatures and L-classes of stratified spaces, Math. Ann. 326 (2003) 589-623 MR

[7] M Banagl, B Chriestenson, Intersection spaces, equivariant Moore approximation and the signature, J. Singul. 16 (2017) 141-179 MR

[8] M Banagl, L Maxim, Deformation of singularities and the homology of intersection spaces, J. Topol. Anal. 4 (2012) 413-448 MR

[9] M Banagl, L Maxim, Intersection spaces and hypersurface singularities, J. Singul. 5 (2012) 48-56 MR

[10] R Bott, L W Tu, Differential forms in algebraic topology, Graduate Texts in Mathematics 82, Springer (1982) MR

[11] J-P Brasselet, G Hector, M Saralegi, Théorème de de Rham pour les variétés stratifiées, Ann. Global Anal. Geom. 9 (1991) 211-243 MR 
[12] D Chataur, M Saralegi-Aranguren, D Tanré, Intersection cohomology, simplicial blow-up and rational homotopy, Mem. Amer. Math. Soc. 1214, Amer. Math. Soc., Providence, RI (2018) MR

[13] J Cheeger, On the spectral geometry of spaces with cone-like singularities, Proc. Nat. Acad. Sci. U.S.A. 76 (1979) 2103-2106 MR

[14] J Cheeger, On the Hodge theory of Riemannian pseudomanifolds, from "Geometry of the Laplace operator" (R Osserman, A Weinstein, editors), Proc. Sympos. Pure Math. 36, Amer. Math. Soc., Providence, RI (1980) 91-146 MR

[15] J Cheeger, Spectral geometry of singular Riemannian spaces, J. Differential Geom. 18 (1983) 575-657 MR

[16] T Essig, About a de Rham complex describing intersection space cohomology in a non-isolated singularity case, Diplomarbeit, Ruprecht-Karls-Universität Heidelberg (2012)

[17] G Friedman, Singular intersection homology, book project (2019) Available at http://faculty.tcu.edu/gfriedman/IHbook.pdf

[18] G Friedman, E Hunsicker, Additivity and non-additivity for perverse signatures, J. Reine Angew. Math. 676 (2013) 51-95 MR

[19] G Friedman, J E McClure, Cup and cap products in intersection (co)homology, Adv. Math. 240 (2013) 383-426 MR

[20] M Goresky, R MacPherson, Intersection homology theory, Topology 19 (1980) 135$162 \mathrm{MR}$

[21] M Goresky, R MacPherson, Intersection homology, II, Invent. Math. 72 (1983) 77129 MR

[22] D Grieser, E Hunsicker, Pseudodifferential operator calculus for generalized $\mathbb{Q}-$ rank 1 locally symmetric spaces, II, in preparation

[23] T Hausel, E Hunsicker, R Mazzeo, Hodge cohomology of gravitational instantons, Duke Math. J. 122 (2004) 485-548 MR

[24] E Hunsicker, Hodge and signature theorems for a family of manifolds with fibre bundle boundary, Geom. Topol. 11 (2007) 1581-1622 MR

[25] E Hunsicker, Extended Hodge theory for fibred cusp manifolds, J. Topol. Anal. 10 (2018) 531-562 MR

[26] H C King, Topological invariance of intersection homology without sheaves, Topology Appl. 20 (1985) 149-160 MR

[27] M Klimczak, Poincaré duality for spaces with isolated singularities, Manuscripta Math. 153 (2017) 231-262 MR

[28] E Kreyszig, Introductory functional analysis with applications, Wiley (1989) MR 
[29] J M Lee, Introduction to smooth manifolds, Graduate Texts in Mathematics 218, Springer (2003) MR

[30] R B Melrose, The Atiyah-Patodi-Singer index theorem, Research Notes in Mathematics 4, A K Peters (1993) MR

[31] M Saralegi-Aranguren, De Rham intersection cohomology for general perversities, Illinois J. Math. 49 (2005) 737-758 MR

[32] S Sasaki, On the differential geometry of tangent bundles of Riemannian manifolds, Tôhoku Math. J. 10 (1958) 338-354 MR

[33] P H Siegel, Witt spaces: a geometric cycle theory for KO-homology at odd primes, Amer. J. Math. 105 (1983) 1067-1105 MR

[34] E H Spanier, Algebraic topology, McGraw-Hill (1966) MR

[35] M Spiegel, $K$-theory of intersection spaces, $\mathrm{PhD}$ thesis, Ruprecht-Karls-Universität Heidelberg (2013) Available at http://doi.org/10.11588/heidok.00015738

Mathematisches Institut, Universität Heidelberg

Heidelberg, Germany

Department of Mathematical Sciences, Loughborough University

Loughborough, United Kingdom

banagl@mathi.uni-heidelberg.de, e.hunsicker@lboro.ac.uk

http://www.mathi.uni-heidelberg.de/ banagl/, https://www.lboro.ac.uk/ departments/maths/staff/academic/eug\%c3\%a9nie-hunsicker/

Proposed: Steve Ferry

Seconded: Frances Kirwan, Ralph Cohen
Received: 20 February 2015

Revised: 4 August 2017 
